\title{
Towards a Complete Description of High-Energy Photoproduction
}

\author{
Gerhard A. Schuler and Torbjörn Sjöstrand \\ Theory Division, CERN \\ CH-1211 Geneva 23 \\ Switzerland
}

\begin{abstract}
A real photon has a dual nature, as a pointlike elementary particle and as a q $\bar{q}$ fluctuation, in the latter case often with hadron-like properties. Therefore high-energy $\gamma p$ interactions have a rich and not yet well explored structure. In this paper, we propose a consistent formulation, which is based on a subdivision of the total cross section into three distinct event classes: direct, VMD and anomalous. The VMD class, in its turn, contains the subclasses familiar from hadron-hadron physics. Within this approach, we calculate all the partial cross sections. Many other aspects of the event classes are also considered, such as proton and photon structure functions, jet rates and beam remnant structures. A major rôle is played by two distinct transverse momentum cut-off scales, which separate the classes. When all the ingredients are put together, we obtain a detailed model for photoproduction events. In order to test this approach, we also propose several alternative scenarios, each of which emphasizes different aspects of the photon. Predictions are presented for HERA events.
\end{abstract}

CERN-TH.6796/93

February 1993 


\section{Introduction}

The photon has a rich structure, which still is poorly explored. Alternative descriptions may be attempted, for the photon itself and for the events that result in the interaction between a real photon and a hadron. We have proposed one approach in Ref. [1], which will be presented in detail in the current paper.

Previous descriptions of $\gamma p$ interactions and their (hadronic) final states can be divided into two classes.

1. The vector-meson-dominance (VMD) approach [2] emphasizes the hadronic component of the photon [3], i.e. that an original 'bare' photon may fluctuate into a vector meson state $V, \gamma \leftrightarrow V$, and undergo interactions (almost) exclusively through its vector-meson component. The main aim of this approach has been the description of soft $\left(\right.$ low- $\left.p_{\perp}\right)$ physics, namely the total $\gamma \mathrm{N}$ cross section and photoproduction of vector mesons. Fixed-target photoproduction is rather well described by VMD, i.e. $\gamma \mathrm{N}$ and $\pi \mathrm{N}$ events are closely similar. Significant differences only appear at the top end of the $p_{\perp}$ range accessed at fixed-target energies [4]. These deviations may be interpreted as evidence for direct interactions of the pointlike bare photon.

2. More recent work is triggered by the prospects of high-energy $\gamma p$ interactions at HERA $[5,6]$. Here the interest focuses on the perturbatively calculable high- $p_{\perp}$ physics, i.e. on the production of jets, high- $p_{\perp}$ single particles, etc. Two kinds of processes contribute to the high- $p_{\perp}$ cross sections: (i) direct photon processes, where the photon couples directly to the partons within the proton, and (ii) resolved photon processes, which describe scatterings between partons in the photon and partons in the proton.

The (QCD-evolved) parton distribution functions (PDFs) of the photon are extracted from two-photon physics [7]. In such a procedure the origin of the photon PDFs is wiped out, i.e. there is no way to tell whether the PDFs describe a hadronlike or a pointlike photon [8]. Based on (scaling) VMD distribution functions, e.g. $F_{2}\left(x, Q^{2}\right)=0.2 \alpha(1-x)$, the prejudice is that the VMD component contributes only to low- $p_{\perp}$ physics $[7]$.

Compared with the time when the VMD concept was originally formulated, our understanding of hadronic physics has increased substantially. Experiments from low-energy fixed-target facilities up to high-energy $\bar{p} p$ colliders reveal a varied structure, ranging from diffractive and soft physics to semihard processes and (rare) high- $p_{\perp}$ events. Though the major fraction of hadronic physics cannot yet be explained from first principles, we do have a successful description that not only achieves a smooth matching of soft and hard physics but also includes details such as the multiple scatterings of partons.

Now, if the photon has a hadronic component, then it will also suffer the same interactions as a hadron. In other words, given the similarity of photon-hadron and hadronhadron interactions at low energies, it is natural to expect $\gamma p$ processes to replicate $p p$ or $\bar{p} p$ ones at comparable high energies, including e.g. the emergence of high- $p_{\perp}$ physics. Therefore, to unravel the nature of the photon, we should rather search for $\gamma p$ characteristics that go beyond those of pp. We are led to the following questions:

- Does the photon behave like a hadron in high-energy scatterings? Do we observe the same pattern of elastic, diffractive, minimum-bias (low- $\left.p_{\perp}\right)$ and high- $p_{\perp}$ events?

- Photon interactions are different from hadron ones, as indicated by jet production in two-photon physics [9] and fixed-target high- $p_{\perp}$ production [4]. How can we define a 'bare' photon? How big is its contribution, e.g. is it really negligible in soft reactions 
as postulated in VMD? What final states does it give?

- Is the direct photon component the only difference? Theoretically, we expect an 'anomalous' contribution, i.e. the partonic content of the photon should differ from that of a $\rho^{0}$. Inclusively, this concerns the PDFs, exclusively, the beam remnants and the hadronic final states.

- If the physical photon can be in various states, how can we represent it without double counting and yet have a continuous matching of the different states and their interactions?

In our approach, we provide a framework that can be used to give first answers to all the above questions. The intuitive photon picture that we have in mind is the following. To first approximation, a real photon is a fundamental pointlike particle. Through its direct couplings to quarks, however, it has the possibility to fluctuate into a quarkantiquark pair, Fig. 1. These $\gamma \leftrightarrow q \bar{q}$ fluctuations may have different virtualities, as roughly characterized by the common $p_{\perp}^{2}$ of the $q$ and $\bar{q}$ with respect to the photon direction. When the virtuality is small the fluctuation is long-lived. There is then time for a cloud of soft gluons to develop around the $q \bar{q}$ pair, and a vector meson wave function description may be a good approximation. This is the $\gamma \leftrightarrow V$ transition postulated in VMD. When the virtuality is larger, the fluctuation is too short-lived to develop into an ordinary hadronic state, although hard gluon emission may still occur. In this latter case, a perturbative description is fully appropriate, while the VMD part of the photon must be based on a non-perturbative phenomenological ansatz. In total, the photon thus has three possible states to be found in: bare, $V$, and perturbative $q \bar{q}$. Correspondingly, $\gamma p$ events may be classified in three distinct classes: direct, VMD and anomalous.

In the following we first develop our picture of the photon and subsequently that of the event classes. To specify our description of $\gamma p$ interactions we must

- derive parametrizations for all the partial (event class) cross sections in the model,

- define the subdivision of the photon structure function into a VMD part and an anomalous part,

- unitarize minijet cross sections, i.e. allow for several parton-parton interactions within one event,

- extend the standard proton structure functions to small $Q^{2}$ and/or small $x$ scales, and

- discuss all further details needed to go from simple parton-level cross sections to hadronic events that can be confronted with data.

The objective is to provide a complete picture of high-energy photon-hadron interactions, in the sense that all event classes of non-negligible cross section are described in reasonable detail.

The development of the model was prompted by the recent HERA workshop [6, 10] and startup, but preceded the appearance of photoproduction results on total cross sections $[11,12]$ and event properties $[13,14]$. The data presented so far, while capable of excluding some extreme scenarios, is still not detailed enough to provide a full understanding of what is going on. In order to promote further studies, we present results for our model and for four alternative scenarios, picked so as to emphasize different aspects of the photon. If these alternatives can be discriminated, based on HERA data, we believe that photon interactions will become better understood.

The model is implemented in the PYTHIA/JETSET programs, where complete events can be generated. Other programs exist [15], but our approach differs from these in many 
respects and is far more complete.

The plan of this paper is as follows. We start with our description of the physical photon in section 2. In section 3 we discuss some aspects of hadron-hadron physics, which we will have to make use of within the context of the VMD model. Section 4 contains a description of our new parametrizations of partial cross sections. This discussion is continued in section 5, where structure functions, jet cross sections and transverse momentum cut-offs are introduced. The main model and the four alternatives are presented in section 6, together with further details of the model. Results for HERA energies are shown in section 7. Finally, some conclusions are given in section 8 .

In all parts of this paper we will assume that the photon is real, i.e. that its virtuality $Q^{2}$ is negligible. For an ep collider, this could be ensured by an electron tagger, e.g. for the region $Q^{2} \leq 0.01 \mathrm{GeV}^{2}$. In the region of larger $Q^{2}$ we expect a gradual breakdown of the $Q^{2} \equiv 0$ photoproduction ansatz, and a continuous transition towards a deep inelastic scattering description.

\section{The Physical Photon}

Phenomenologically, photon-hadron interactions bear many remarkable similarities with purely hadronic ones [3]. This can be explained if the physical photon is a superposition of a bare photon $\left|\gamma_{B}\right\rangle$ and a hadronic component $\sqrt{\alpha}|h\rangle$,

$$
|\gamma\rangle=\sqrt{Z_{3}}\left|\gamma_{B}\right\rangle+c \sqrt{\alpha}|h\rangle
$$

where $Z_{3}=1-c^{2} \alpha$ assures the proper normalization of $|\gamma\rangle$ and $c$ is of $\mathcal{O}(1)$. The state $|h\rangle$ must have the same quantum numbers as the photon, $J^{P C}=1^{--}, Q=B=S=0$, i.e. be a flavour-diagonal vector meson. The aforementioned similarities between $\gamma \mathrm{N}$ and $h \mathrm{~N}$ interactions imply that, at high energies, $\gamma_{B} \mathrm{~N}$ interactions can only account for a small fraction of the total $\gamma \mathrm{N}$ ones: the smallness of the $\sqrt{\alpha}|h\rangle$ component is more than compensated by an intrinsically much larger $h \mathrm{~N}$ cross section.

The hypothesis of VMD is that the bare component $\left|\gamma_{B}\right\rangle$ cannot interact at all with hadrons. In its simplest formulation, VMD asserts moreover that the three vector mesons $\rho^{0}, \omega$, and $\phi$ are the sole hadronic constituents of the photon, based on the observation of their copious photoproduction. This gives

$$
c \sqrt{\alpha}|h\rangle=\sum_{V=\rho^{0}, \omega, \phi} \frac{e}{f_{V}}|V\rangle .
$$

The inclusion of heavier constituents is referred to as generalized vector dominance (GVD).

The VMD/GVD formulations are intended to be applicable, with trivial extensions, also for timelike and spacelike photons. However, the behaviour of the cross section for $\mathrm{e}^{+} \mathrm{e}^{-} \rightarrow$ hadrons, $\sigma_{\text {had }}^{\mathrm{e}^{+}}(s)$, cannot be accommodated in VMD. In GVD an abundant spectrum of higher-mass states is required, which creates problems in the description of deep inelastic scattering. The improved parton model, on the other hand, nicely predicts

$\sigma_{\text {had }}^{\mathrm{e}^{+}}(s)$ from rather small energies onwards. In the deep inelastic scattering (DIS) régime it is difficult to obtain a good VMD/GVD description of the scaling phenomenon. There again a parton description is more attractive. Augmented by perturbative QCD, also the logarithmic deviations from scaling are well explained.

On the other hand, the small- $x$ and/or small- $Q^{2}$ region (where $x$ denotes the longitudinal parton momentum in the infinite momentum frame and $Q^{2}$ the photon virtuality) 
is beyond the range of validity of the parton model [16]. This 'Regge domain' can be described (at least phenomenologically) in the framework of a hadronic photon. It was thus conjectured long ago [17] that the two approaches, VMD and the parton model, are in fact compatible with and complementary to each other. In this paper, we aim at such a marriage of the two descriptions:

1. We start with simple VMD, i.e. restrict the vector meson dominance model to the lightest states $\rho^{0}, \omega$, and $\phi$. We emphasize that this is pure phenomenology, which, however, provides a successful description of photoproduction at low energies.

2. For the higher-mass contributions we switch from the hadron language to the parton language. That is, rather than extending the sum in eq. (2) over heavier meson states, as is done in GVD, we represent a high-mass hadronic fluctuation of the photon by a perturbatively calculable quark-antiquark state $|q \bar{q}\rangle$. By virtue of QCD corrections, states with additional gluons and $q \bar{q}$ pairs are included: $|q \bar{q} g\rangle$, $|q \bar{q} g g\rangle,|q \bar{q} q \bar{q}\rangle$, etc.

3. Finally we demand a non-vanishing bare photon-nucleon interaction, quite in contrast to VMD, where such interactions are absent. It is well known that the lowmass, VMD contributions account for only about $80 \%$ of the observed total $\gamma p$ cross section [3] (see section 4.2). In GVD, the missing contribution is attributed to heavier meson states. In our approach, the high-mass contribution is calculated perturbatively and, in fact, does not fully explain the missing difference. We are therefore led to a significant bare-photon interaction rate.

We define the bare-photon interactions as the interactions of the photon with partons in the proton. At large spacelike virtualities of the photon $\left(Q^{2} \geq 5 \mathrm{GeV}^{2}\right)$ such interactions, in fact, define the parton model of DIS. But in the photoproduction limit $Q^{2} \rightarrow 0$ also the $F_{2}^{e m}\left(x, Q^{2}\right) \rightarrow 0$, where $F_{2}^{e m}\left(x, Q^{2}\right)=\sum_{a=q, \bar{q}} e_{\mathrm{q}}^{2} x f_{a}^{\mathrm{p}}\left(x, Q^{2}\right)$ in the parton model. It is therefore clear that we shall have to modify the PDFs if we want to maintain a partonic view of the proton all the way to the photoproduction limit (see section 5.1).

One should be aware of some basic problems in the description of the photon. In a $\gamma p$ event, physical observables are calculated in terms of a convolution of an internal photon structure, an internal proton structure and a mutual interaction. Often what is put where is convention. For instance, in the standard description of deep inelastic scattering, the photon and its interaction are taken to be pointlike by definition. Any influence of $\gamma \leftrightarrow V$ fluctuations is put into the concept of proton structure functions, under the general heading of higher-twist effects. Uncertainties of this kind will have to be borne in mind when assessing any photoproduction model.

Our ansatz is to split the spectrum of hadronic fluctuations of the photon into a low- and high-mass part, just as one is used to split the description of $\sigma_{\text {had }}^{\mathrm{e}^{+} \mathrm{e}^{-}}(s)$ into one resonance and one continuum region. The former part is based on phenomenology, the latter on perturbative QCD. Here uncertainties arise mainly for the following three reasons. Firstly, the low-mass part may contain more structure than is described by the discrete sum over three orthogonal vector mesons, either by a continuum contribution underneath the resonance Breit-Wigner shapes or by off-diagonal couplings between $\rho^{0}$, $\omega$ and $\phi$. Secondly, perturbative QCD might not be valid down to the rather low scale at which the 'high'-mass part starts, which in our model will turn out to have to be around $m_{\phi} \sim 1 \mathrm{GeV}$. Finally, interference effects between the two regions are neglected. It is only experiment that can tell whether it is justified to split the mass spectrum in two non-commuting parts as described above. 
We now arrive at the following representation of the physical photon

$$
|\gamma\rangle=\sqrt{Z_{3}}\left|\gamma_{B}\right\rangle+\sum_{V=\rho^{0}, \omega, \phi} \frac{e}{f_{V}}|V\rangle+\frac{e}{f_{\mathrm{q}} \overline{\mathrm{q}}}|\mathrm{q} \overline{\mathrm{q}}\rangle,
$$

where

$$
Z_{3}=1-\sum_{V=\rho^{0}, \omega, \phi}\left(\frac{e}{f_{V}}\right)^{2}-\left(\frac{e}{f_{\mathrm{q} \overline{\mathrm{q}}}}\right)^{2}
$$

(see also section 5.2).

In order that the above superposition is smooth and free of double counting, we have to introduce scales that separate the three components. The main one is $p_{0}$, which separates the low-mass from the high-mass region, $p_{0} \approx m_{\phi} / 2 \approx 0.5 \mathrm{GeV}$. Since it is the same $\gamma q \bar{q}$ vertex that is responsible for the bare $\gamma p$ interactions, $p_{0}$ is also the lower cut-off of the photon-parton cross sections. (We are confirmed in our ansatz by the observation that this value of $p_{0}$ results in the correct size of the bare photon contribution to the total $\gamma p$ cross section, see section 5.4).

The factor $\left(e / f_{V}\right)^{2}=4 \pi \alpha / f_{V}^{2}$ gives the probability for the transition $\gamma \rightarrow V$. The coefficients $f_{V}^{2} / 4 \pi$ are taken to be 2.20 for $\rho^{0}, 23.6$ for $\omega$, and 18.4 for $\phi$, based on lowenergy fits [3]. An element of uncertainty is that the $f_{V}^{2}$ coefficients come out differently depending on whether they are extracted from $\mathrm{e}^{+} \mathrm{e}^{-}$annihilation $\left(\bar{f}_{V}^{2}\right)$ or from photoproduction of vector mesons $\left(\hat{f}_{V}^{2}\right)$; what we use is the geometrical mean $\left(\sqrt{\bar{f}_{V}^{2} \hat{f}_{V}^{2}}\right)$. Normally, the $f_{V}^{2}$ are assumed to be energy-independent.

One cannot expect the superposition in eq. (3) to occur in fixed ratios for all processes, however. Different $\gamma \mathrm{N}$ interactions involve different time scales and thus different probabilities for the photon to be in a specific state. The $f_{V}$ couplings quoted above imply that the photon can be found in its VMD state about $0.4 \%$ of the time, but the contribution from the 'anomalous' high-mass fluctuations depends on the typical scale $\mu$ of the interaction

$$
\left(\frac{e}{f_{\mathrm{q} \overline{\mathrm{q}}}}\right)^{2} \approx \frac{\alpha}{2 \pi} \frac{N_{C}}{3}\left(2 \sum_{\mathrm{q}} e_{\mathrm{q}}^{2}\right) \ln \left(\frac{\mu^{2}}{p_{0}^{2}}\right),
$$

where $N_{C}=3$ and q runs over the quarks that can be taken massless compared with $\mu$. The logarithmic increase with $\mu$ implies that the anomalous contribution to the total photoproduction cross section $\left(\mu \sim m_{V}\right)$ is less important than that to high- $p_{\perp}$ jet production $\left(\mu \sim p_{\perp}\right)$. Most of the time a photon is still a point particle, $1-Z_{3} \ll 1$. It is only because the hadronic $\rho^{0} \mathrm{p}$ cross section is so much larger than the direct $\gamma p$ one that most $\gamma p$ cross sections are dominated by the VMD and anomalous contributions.

A fourth term in the photon wave function is $\left|\ell^{+} \ell^{-}\right\rangle$, where $\ell=e, \mu$ or $\tau$. Its contribution to $1-Z_{3}$ is entirely perturbatively calculable, like eq. (5) with $N_{C}=1, e_{\mathrm{q}} \rightarrow e_{\ell}=-1$ and $p_{0} \rightarrow m_{\ell}$. Since the leptonic states would not undergo strong interactions to leading order, they can be safely neglected in the following.

By virtue of the superposition in eq. (3) we are led to the model of $\gamma p$ interactions [1], where three different kinds of events may be distinguished:

- Direct events, Fig. 2a, wherein the photon interacts directly with a parton from the proton. The process is perturbatively calculable, and no photon structure function is involved. The typical event structure is two high- $p_{\perp}$ jets and a proton remnant, while the photon does not leave behind any remnant. 
- VMD events, Fig. 2b, in which the photon fluctuates into a vector meson, predominantly a $\rho^{0}$. All the event classes known from ordinary hadron-hadron interactions may thus occur here, such as elastic, diffractive, low- $p_{\perp}$ and high- $p_{\perp}$ events. For the latter, one may define a (VMD) photon structure function, and the photon also leaves behind a beam remnant. This remnant is smeared in transverse momentum by a typical 'primordial $k_{\perp}$ ' of a few hundred MeV.

- Anomalous events, Fig. 2c, in which the photon fluctuates into a q $\bar{q}$ pair of larger virtuality than in the VMD class. This process is perturbatively calculable, as is the subsequent QCD evolution. It gives rise to the so-called anomalous part of the photon structure function, whence our name for the class. Only high- $p_{\perp}$ events may occur. Either the $q$ or the $\bar{q}$ plays the rôle of a beam remnant, but this remnant has a larger $p_{\perp}$ than in the VMD case, related to the virtuality of the $\gamma \leftrightarrow q \bar{q}$ fluctuation.

In terms of cross sections, eq. (3) therefore corresponds to

$$
\sigma_{\text {tot }}^{\gamma \mathrm{p}}(s)=\sigma_{\text {dir }}^{\gamma \mathrm{p}}(s)+\sigma_{\mathrm{VMD}}^{\gamma \mathrm{p}}(s)+\sigma_{\text {anom }}^{\gamma \mathrm{p}}(s) .
$$

This decomposition will be essential for the rest of the discussion.

It should be noted that the above subdivision is not the conventional one. Usually the VMD and the anomalous contributions are joined into one single photon structure function, which is used to calculate the rate of high- $p_{\perp}$ events, so-called resolved events. (Exceptions exist, see e.g. ref. [18].) The VMD event class then explicitly excludes high- $p_{\perp}$ events, i.e. is mainly made up of low- $p_{\perp}$ events. There are several reasons why we avoid the conventional approach. Firstly, this approach does not respect the known similarities between photoproduction and hadroproduction. Secondly, it is not possible to smoothly join soft and hard $\gamma p$ interactions, owing to the lack of a physical picture of the photon such as eq. (3). Thirdly, it is inconsistent to invoke VMD to describe e.g. diffractive reactions such as $\gamma \mathrm{p} \rightarrow \rho^{0} X$, but neglect the partonic content of the vector mesons. Finally, we expect VMD and anomalous high- $p_{\perp}$ events to be different, e.g. with respect to beam remnant structure. Therefore they should not be joined in a resolved photon structure function.

We finally point out our prediction of two fundamental scales in $\gamma$ p interactions, in contrast with hadronic interactions where there is just one. It is the two transverse momentum cut-off scales, here called $p_{0}$ and $p_{\perp \text { min }}$.

- The $p_{0}$ cut-off, already discussed, is to be applied to the $\gamma \leftrightarrow q \bar{q}$ vertex present in all three event classes. When $p_{\perp}<p_{0}$ the $q \bar{q}$ pair is assumed to build up a vector meson, and we are back in the VMD model. Direct and anomalous events therefore are characterized by $p_{\perp}>p_{0}$.

- The $p_{\perp \text { min }}$ cut-off, in the ballpark of $1.3 \mathrm{GeV}$, defines the minimum $p_{\perp}$ of hard parton-parton interactions in hadronic events. It is assumed that below this scale an exchanged gluon has a transverse wavelength that is larger than a typical hadronic size, and that the gluon therefore only observes net colour neutral hadrons and decouples. This cut-off is determined from $\bar{p} p$ data and is directly applicable to VMD events. A corresponding cut-off is also needed for anomalous events, but we here come up with a somewhat higher value, say $2.2 \mathrm{GeV}$, from $\gamma$ p cross section considerations. This could be interpreted as a smaller spatial extent for the anomalous class of $q \bar{q}$ fluctuations than for the VMD one.

For practical reasons both $p_{0}$ and $p_{\perp \text { min }}$ are taken as sharp cut-offs, although the picture we have in mind is one of smooth turn-offs at some characteristic scale. 


\section{Hadronic Physics}

\subsection{Hadronic event classes}

In hadronic physics, one is used to distinguish between several different event classes, such as elastic, diffractive and non-diffractive events. These classes then carry over to a description of the VMD component of the photon interactions.

The standard elastic process $V+\mathrm{p} \rightarrow V+\mathrm{p}$ here generalizes to the 'VMD elastic' process

$$
\gamma+\mathrm{p} \rightarrow V+\mathrm{p}
$$

The 'truly elastic' (Compton) process $\gamma+p \rightarrow \gamma+p$ is of $\mathcal{O}\left(\alpha^{2}\right)$, compared with the $\mathcal{O}(\alpha)$ VMD processes, and can safely be neglected in the following.

The VMD single and double diffractive processes correspondingly are

$$
\begin{aligned}
& \gamma+\mathrm{p} \rightarrow V+X \\
& \gamma+\mathrm{p} \rightarrow X+\mathrm{p} \\
& \gamma+\mathrm{p} \rightarrow X_{1}+X_{2}
\end{aligned}
$$

Further diffractive event topologies are possible (central diffraction etc.), but these are believed to be less important and are neglected in the following.

The non-diffractive event class covers everything else, i.e. the generic process

$$
\gamma+\mathrm{p} \rightarrow X
$$

where the system $X$ is supposed not to contain any large rapidity gaps, i.e. to be distinctive from diffractive events. The non-diffractive component is often referred to as "minimumbias' events, although an experimentally defined minimum-bias sample is likely to contain some contamination from diffractive events.

The non-diffractive cross section for a generic process $A+B \rightarrow$ anything is defined from the equation

$$
\sigma_{\text {tot }}^{A B}(s)=\sigma_{e l}(s)+\sigma_{s d(A X)}(s)+\sigma_{s d(X B)}(s)+\sigma_{d d}(s)+\sigma_{n d}(s),
$$

once the other terms are known. Here $e l$ stands for elastic, $s d(A X)$ and $s d(X B)$ for single diffractive on the two sides, $d d$ for double diffractive and $n d$ for non-diffractive.

\subsection{Minimum-bias events}

The non-diffractive, inelastic part $\sigma_{n d}(s)$ of the cross section contains both events with high- $p_{\perp}$ ('hard') jets and events without any visible hard jet activity.

To lowest order, the hard jet production comes from $2 \rightarrow 2$ processes such as $q+q^{\prime} \rightarrow$ $q+q^{\prime}, q+g \rightarrow q+g$ and $g+g \rightarrow g+g$. The differential event rate, as a function of the jet $p_{\perp}$, is given by

$$
\frac{\mathrm{d} \sigma_{i n t}}{\mathrm{~d} p_{\perp}^{2}}=\sum_{i, j, k} \int \mathrm{d} x_{1} \int \mathrm{d} x_{2} \int \mathrm{d} \hat{t} f_{i}\left(x_{1}, \mu^{2}\right) f_{j}\left(x_{2}, \mu^{2}\right) \frac{\mathrm{d} \hat{\sigma}_{i j}^{k}}{\mathrm{~d} \hat{t}} \delta\left(p_{\perp}^{2}-\frac{\hat{t} \hat{u}}{\hat{s}}\right),
$$

where $i$ and $j$ label possible incoming partons taken from the two incoming particles, and $k$ different allowed final-state flavour contents. In this parton level language, each event 
contains two jets with opposite and compensating $p_{\perp}$; the inclusive jet rate therefore is a factor of 2 larger. As scale in structure functions we conventionally use $\mu^{2}=p_{\perp}^{2}$. (Normally the scale is denoted $Q^{2}$, but we prefer $\mu^{2}$ in this paper, since $Q^{2}$ is already used to denote the virtuality of the photon.)

If one introduces a cut-off $p_{\perp \text { min }}$, the integrated rate above this cut-off is given by

$$
\sigma_{\text {hard }}\left(p_{\perp \text { min }}\right)=\int_{p_{\perp m i n}^{2}}^{s / 4} \frac{\mathrm{d} \sigma_{i n t}}{\mathrm{~d} p_{\perp}^{2}} \mathrm{~d} p_{\perp}^{2} .
$$

For a reasonably large $p_{\perp \text { min }}$ value, one could then subdivide minimum-bias events into a part $\sigma_{\text {hard }}\left(p_{\perp \text { min }}\right)$ of events with hard jets, and a part $\sigma_{\text {soft }}\left(p_{\perp \text { min }}\right)=\sigma_{n d}-\sigma_{\text {hard }}\left(p_{\perp \text { min }}\right)$ of 'low- $p_{\perp}$ ' events, without hard jet activity.

Since the differential jet cross section diverges roughly like $\mathrm{d} p_{\perp}^{2} / p_{\perp}^{4}, \sigma_{\text {hard }}$ is also divergent for $p_{\perp \text { min }} \rightarrow 0$. For a small $p_{\perp \text { min }}$ value, this would lead to $\sigma_{\text {hard }}\left(p_{\perp \text { min }}\right)>\sigma_{n d}(s)$ and $\sigma_{\text {soft }}\left(p_{\perp \text { min }}\right)<0$, which seems to be a contradiction. However, one must remember that $\sigma_{\text {hard }}$ gives the parton-parton cross section. Each of the incoming hadrons may be viewed as a beam of partons, with the possibility of having several parton-parton interactions when the hadrons pass through each other. In this language, $\langle n\rangle=\sigma_{\text {hard }}\left(p_{\perp \text { min }}\right) / \sigma_{n d}(s)$ is simply the average number of parton-parton scatterings above $p_{\perp \text { min }}$ in an event, and this number may well be larger than unity. The mathematical framework, which distributes a varying number of interactions per event and ensures $\sigma_{\text {soft }} \geq 0$, is traditionally called unitarization.

A number of problems would still arise if one tried to push $p_{\perp \text { min }}$ all the way to zero. However, it is likely that some effective cut-off should be introduced simply because the incoming hadrons are colour neutral objects: when the $p_{\perp}$ of an exchanged gluon is made small and the transverse wavelength correspondingly large, the gluon can no longer resolve the individual colour charges, and then the effective coupling is decreased. This mechanism cannot be predicted by perturbative QCD but is also not in contradiction with perturbative QCD calculations, which are always performed assuming scattering of free partons (rather than partons inside hadrons). Therefore $p_{\perp \text { min }}$ has to be considered as a free parameter.

A first determination of $p_{\perp \text { min }}$ was done in ref. [19]. Based on studies of multiplicity distributions in $\bar{p} p$ collisions, a value in the range $1.5-2.0 \mathrm{GeV}$ is obtained. For the current work, we made a refit to $\bar{p} p$ collider data [20], using the modified proton structure functions to be discussed in section 5.1. In order to agree with the average charged multiplicity of minimum-bias events, one needs to set $p_{\perp \text { min }} \approx 1.3 \mathrm{GeV}$ at $200 \mathrm{GeV}$ and $\approx 1.45 \mathrm{GeV}$ at $900 \mathrm{GeV}$. It is not clear whether the difference is significant, but in the following we have assumed a slow logarithmic variation

$$
p_{\perp \text { min }}=1.3+0.15 \frac{\ln \left(E_{c m} / 200\right)}{\ln (900 / 200)} .
$$

Similar (constant) values have been determined when the increase of the $\bar{p} p$ total cross section with energy is interpreted as a consequence of the increasing minijet cross section [21]. This interpretation requires additional assumptions compared with a simple multiple interactions scenario, such as

(i) the value of $\sigma_{s o f t}(s)$ before unitarization,

(ii) the shape of the hadronic wave function, and

(iii) the matching with some different physics in the low-energy region, where $\sigma_{\text {tot }}$ is 
decreasing with energy.

Therefore it is convenient to factorize the problem into one of determining $\sigma_{\text {tot }}(s)$, and another of modelling multiple interactions for a given $\sigma_{n d}(s)$, even if the two ultimately may be related. In this paper we shall only be concerned with the second aspect.

For the detailed modelling, we use the formalism described in ref. [19]. A few different model variations are described there. In the simpler version, interactions at different $p_{\perp}$ values are assumed to take place independently of each other, with a probability distribution given by

$$
\mathcal{P}\left(p_{\perp}\right)=\frac{1}{\sigma_{n d}(s)} \frac{\mathrm{d} \sigma_{i n t}}{\mathrm{~d} p_{\perp}} .
$$

This gives a Gaussian distribution in the number of interactions that take place in a given event. In particular, with an average number $\langle n\rangle$ of interactions per event, a fraction $\exp (-\langle n\rangle)$ of events will have no hard interactions at all, and thus be 'true' low- $p_{\perp}$ ones, with beam jets and nothing else.

In a more sophisticated scenario, interactions are assumed to take place with varying impact parameter. Central collisions are then assumed to lead to enhanced activity, while peripheral ones give reduced activity. While roughly preserving the average behaviour, it is thus possible to increase the fluctuations around this average. There is some evidence for a picture of this kind, but throughout this paper we have used the above simpler scenario. It will be interesting to study the importance of different multiple-interaction scenarios for photoproduction physics, as discussed briefly in the next subsection, but to perform all the current calculations for several alternatives would have carried too far.

Further details follow exactly the same pattern as described in refs. [19, 22, 23], and we do not repeat them here. It should be noted, however, that many uncertainties do exist in the modelling. For instance, a proper treatment would require the introduction of a joint structure function for finding a parton $i_{1}$ at $x_{1}$ and a parton $i_{2}$ at $x_{2}$ inside one and the same hadron; what is used is a simple factorized ansatz, with minimal modifications to ensure momentum conservation. Our hope is that many of these uncertainties effectively can be shuffled into the choice of the free parameters of the model, such as $p_{\perp \text { min }}$.

\subsection{Minimum-bias phenomenology}

One of the main surprises of the SpppS collider was the observation that scaled charged multiplicity distributions are getting broader with energy [20]: for the probability distribution of the $z=n_{c h} /\left\langle n_{c h}\right\rangle$ variable one would have expected either a roughly energy-independent distribution ('KNO scaling' [24]) or a shrinking one (Poissonian statistics). At high energies the deviation from a Poissonian distribution is very significant; for $E_{c m}=900 \mathrm{GeV}$ one has $\left\langle n_{c h}\right\rangle=35.6$ and $\sigma\left(n_{c h}\right)=19.5$, while Poissonian statistics would have predicted $\sigma\left(n_{c h}\right)=\sqrt{\left\langle n_{c h}\right\rangle}=6.0$.

This is to be viewed in the light of our experience from $\mathrm{e}^{+} \mathrm{e}^{-}$annihilation. There, approximate KNO scaling seems to hold, but this can fully be understood as a combination of a non-perturbative fragmentation that is essentially Poissonian (or, in fact, a bit narrower, as a consequence of energy-momentum conservation effects) with a perturbative QCD parton shower that has a rough KNO scaling. Hard processes and parton showers are expected to play a less prominent rôle in $\bar{p}$ p events, since hard parton-parton interactions are rare, and only involve a fraction of the energy of the event. It would therefore 
have been natural to expect the Poissonian fragmentation process to be more prominent, and scaled multiplicity distributions to shrink with energy.

Some other mechanism must be found to explain the data, and the prime candidate is the multiple interactions described above. With a varying number of semisoft interactions, a varying number of fragmenting subsystems (strings) are produced. Even if each of these obeys Poissonian statistics by itself (approximately, for fixed string mass), the total distribution may well be much broader, in reasonable agreement with the data. In particular, the high-multiplicity tail comes from events with several interactions.

Also many other interesting observations have been made, which lend support to a scenario with multiple parton-parton interactions [19]. Since we expect that several of these studies can be repeated in photoproduction, we mention a few here. This does not necessarily mean that the same results should be expected in $\gamma p$ as in $\bar{p} p$ collisions, since the direct and anomalous event classes may be rather different from the VMD one, as we shall see.

If events are divided into two hemispheres, called forward $(y>0)$ and backward $(y<0)$, then large forward-backward multiplicity correlations can be observed [25]. These correlations are long-range, i.e. survive also if a central rapidity gap is introduced between the two measurement regions. In a Poissonian fragmentation picture this is not expected. The conclusion would be that some global event characteristic exists, which influences particle distribution at all rapidities in the same way, more or less. The number of interactions in an event would be one such characteristic, and indeed a multiple-interactions scenario can describe the data.

The 'pedestal effect' implies that events with jet activity also have larger activity away from the jet core [26]. The increase of transverse energy away from the jet is marked when the jet $E_{\perp}$ increases from about 3 to $12 \mathrm{GeV}$; thereafter a plateau is reached. This phenomenon may be understood in a multiple-interactions scenario with varying impact parameters. Central collisions are more likely to lead to enhanced activity, both of trigger jets and of multiple interactions in general. Events with hard jets therefore are biased towards central collisions, and naturally contain more underlying activity. The amount of bias is a function of $E_{\perp j e t}$, and nicely saturates at $E_{\perp j e t} \approx 12 \mathrm{GeV}$ [19].

The average transverse momentum of charged particles displays a characteristic increase as a function of the total multiplicity in events [27]. An almost multiplicityindependent $\left\langle p_{\perp}\right\rangle$ is obtained from ordinary fragmentation; the additional $p_{\perp}$ comes from the minijet activity, which is especially important for events with large multiplicity. The observed increasing trend therefore comes out naturally, although the precise shape of $\left\langle p_{\perp}\right\rangle\left(n_{c h}\right)$ is sensitive to the details of the multiple-interactions model.

Direct evidence for multiple interactions may be searched for in events with four jets. The multiple interaction contribution of two $2 \rightarrow 2$ processes is expected to give two pairs of jets with opposite and compensating $p_{\perp}$, unlike standard QCD $2 \rightarrow 4$ processes. However, for experimentally defined jets the QCD 'background' is still very large, so tests are not straightforward. To date AFS and CDF have reported signals, while UA2 prefers to quote an upper limit, although their data are consistent with a signal of the expected order [28]. Because of the difficulty of the tests, the matter cannot be considered as entirely settled. 


\section{Inclusive Cross Sections}

\subsection{The total cross section}

Several different theoretical frameworks can be used to predict the energy variation of the total cross section in various processes. For our study we need a simultaneous description of $\mathrm{pp}, \pi^{ \pm} \mathrm{p}, \mathrm{K}^{ \pm} \mathrm{p}$, and $\gamma \mathrm{p}$ total cross sections made within the same framework, because we want to consider also the differences between these cross sections. This excludes e.g. the parametrizations of the PDG [29], which are based on an unphysical ansatz and do not yield reasonable differences. In the following, we will make use of a Regge-theory [30] inspired model, specifically the recent fits in Ref. [31]. These fits are given in the very economical form

$$
\sigma_{\text {tot }}^{A B}=X^{A B} s^{\epsilon}+Y^{A B} s^{-\eta}
$$

for a process $A+B \rightarrow X$. The powers $\epsilon$ and $\eta$ are universal, with fit values

$$
\epsilon \approx 0.0808, \quad \eta \approx 0.4525
$$

while $X_{A B}$ and $Y_{A B}$ are process-dependent. The first term, the pomeron one, gives the asymptotic rise of the cross section. Ultimately, this increase violates the Froissart-Martin bound [32]; $\epsilon$ should therefore be thought of as slowly decreasing with energy (owing to multi-pomeron exchange effects), although data at current energies are well fitted by a constant $\epsilon$. The second term, the reggeon one, is mainly of interest at low energies.

For $\gamma$ p events, a best fit to data below $20 \mathrm{GeV}$ gives

$$
\sigma_{\text {tot }}^{\gamma \mathrm{p}}(s)=67.7 s^{\epsilon}+129 s^{-\eta}
$$

where $s$ is expressed in $\mathrm{GeV}^{2}$ and $\sigma_{\text {tot }}^{\gamma \mathrm{p}}$ in $\mu \mathrm{b}$. The predicted value at $200 \mathrm{GeV}$ is $160 \mu \mathrm{b}$. This is in good agreement with the reported numbers, $154 \pm 16 \pm 32 \mu \mathrm{b}$ for ZEUS [11] and $159 \pm 7 \pm 20 \mu \mathrm{b}$ for $\mathrm{H} 1$ [12], at about the same energy. In this paper, we will therefore assume that eq. (19) indeed gives the correct $\gamma$ p total cross section.

This approach does not predict the relative composition of events, however.

\subsection{The VMD total cross section}

Within the diagonal VMD model, the $\gamma p$ cross section is predicted by

$$
\sigma_{\mathrm{VMD}}^{\gamma \mathrm{p}}(s)=\sum_{V=\rho^{0}, \omega, \phi} \frac{4 \pi \alpha}{f_{V}^{2}} \sigma_{t o t}^{V \mathrm{p}}(s) .
$$

The $V$ p cross sections may be parametrized as

$$
\begin{aligned}
\sigma_{\text {tot }}^{\rho^{0} \mathrm{p}} \approx \sigma_{\text {tot }}^{\omega \mathrm{p}} & \approx \frac{1}{2}\left(\sigma_{\text {tot }}^{\pi^{+} \mathrm{p}}+\sigma_{\text {tot }}^{\pi^{-} \mathrm{p}}\right) \approx 13.63 s^{\epsilon}+31.79 \mathrm{~s}^{-\eta} \\
\sigma_{\text {tot }}^{\phi \mathrm{p}} & \approx \sigma_{\text {tot }}^{\mathrm{K} \mathrm{p}}+\sigma_{\text {tot }}^{\mathrm{K}-\mathrm{p}}-\sigma_{\text {tot }}^{\pi^{-} \mathrm{p}} \approx 10.01 s^{\epsilon}-1.51 \mathrm{~s}^{-\eta}
\end{aligned}
$$

in units of $\mathrm{mb}$ (for convenience, hadron-hadron cross sections are given in $\mathrm{mb}$ and $\gamma \mathrm{p}$ ones in $\mu \mathrm{b}$ ). The equations are based on the additive quark model [33] and on $\pi \mathrm{p}$ and Kp fits [31]. For the second equation one also needs to assume that the parton-hadron cross sections $\sigma_{\text {tot }}^{\mathrm{up}} \approx \sigma_{\text {tot }}^{\mathrm{dp}}$. 
Putting these numbers together, one obtains

$$
\sigma_{\mathrm{VMD}}^{\gamma \mathrm{p}} \approx 53.4 s^{\epsilon}+115 s^{-\eta}
$$

Roughly speaking, this is the $\rho^{0}$ p cross section scaled down by a factor 250 . Comparing with eq. (19), one finds that the VMD part corresponds to about $80 \%$ of the total $\gamma p$ cross section, Fig. 3. (Since the coefficients of the $s^{\epsilon}$ and $s^{-\eta}$ terms are not perfectly matched, the ratio varies with c.m. energy; the low-energy limit is $89 \%$ and the high-energy one $79 \%$.) The remaining part of $\sigma_{\text {tot }}^{\text {p }}$ is attributed to the direct and anomalous event classes:

$$
\sigma_{\text {dir }}^{\gamma \mathrm{p}}+\sigma_{\text {anom }}^{\gamma \mathrm{p}}=\sigma_{\text {tot }}^{\gamma \mathrm{p}}-\sigma_{\mathrm{VMD}}^{\gamma \mathrm{p}} \approx 14.3 s^{\epsilon}+14 s^{-\eta}
$$

These formulae should not be viewed uncritically. It is not at all clear that a pomeron/reggeon picture of the VMD photon is appropriate, nor that the additive quark model can be used [34]. The greatest mystery, however, is why the sum of the direct and anomalous cross sections should be describable by the form $X s^{\epsilon}+Y s^{-\eta}$, when the two processes involve neither pomeron nor reggeon exchange. It is not impossible that the parametrization of eq. (24) turns out to be fortuitous, and only works over some limited range of energies. Then also eq. (19) could have to be modified.

\subsection{The VMD elastic cross section}

The optical theorem relates the elastic and total cross sections

$$
\left.\frac{\mathrm{d} \sigma_{e l}}{\mathrm{~d} t}\right|_{t=0}=\frac{1+\rho^{2}}{16 \pi} \sigma_{t o t}^{2} .
$$

In the following we assume a vanishing ratio of the real to imaginary part of the forward scattering amplitude, $\rho \approx 0$, which is supported by existing data. Further, we use the simple ansatz

$$
\frac{\mathrm{d} \sigma_{e l}}{\mathrm{~d} t} \propto e^{B_{e l} t}
$$

This is appropriate for low $|t|$, where the bulk of the cross section is, but does not reproduce the full complexity of the high- $|t|$ behaviour. With these two simplifications, the optical theorem reduces to

$$
\sigma_{e l} \approx \frac{1}{16 \pi} \frac{\sigma_{t o t}^{2}}{B_{e l}} \approx\left(0.0511 \mathrm{mb}^{-1} \mathrm{GeV}^{-2}\right) \frac{\sigma_{t o t}^{2}}{B_{e l}}
$$

In the last step, we have used the standard conversion formula $1 \mathrm{GeV}^{-2} \approx 0.3894 \mathrm{mb}$.

From this, the VMD elastic $\gamma+p \rightarrow V+p$ cross section can be derived as in eq. (20), i.e.

$$
\sigma_{e l}^{\gamma \mathrm{p}}(s)=\sum_{V=\rho^{0}, \omega, \phi} \frac{4 \pi \alpha}{f_{V}^{2}} \sigma_{e l}^{V \mathrm{p}}(s) \approx \sum_{V=\rho^{0}, \omega, \phi} \frac{4 \pi \alpha}{f_{V}^{2}} \frac{1}{16 \pi} \frac{\left(\sigma_{t o t}^{V \mathrm{p}}(s)\right)^{2}}{B_{e l}(s)} .
$$

Note that only one factor of $4 \pi \alpha / f_{V}^{2}$ should be used; it is for the truly elastic process $(\gamma \rightarrow V)+\mathrm{p} \rightarrow(V \rightarrow \gamma)+\mathrm{p}$ that two such factors appear. 
In the pomeron exchange picture, the slope parameter $B_{e l}$ is predicted to be

$$
B_{e l}^{A B}(s)=2 b_{A}+2 b_{B}+2 \alpha^{\prime} \ln \left(\alpha^{\prime} s\right) .
$$

Here $b_{A}$ is the slope of the form factor for particle $A, b_{\mathrm{p}} \approx 2.3 \mathrm{GeV}^{-2}, b_{\pi} \approx 1.4 \mathrm{GeV}^{-2}$. Based on the VMD philosophy, we assume $b_{\gamma} \approx b_{\rho} \approx b_{\pi}$. The $\alpha^{\prime}$ is the slope of the pomeron trajectory, $\alpha_{\mathbb{P}}(t) \approx 1+\epsilon+\alpha^{\prime} t, \alpha^{\prime} \approx 0.25 \mathrm{GeV}^{-2}$. The choice of $\alpha^{\prime} s$ as argument in the logarithm of eq. (29) is a matter of convention; any multiple of $s$ could have been used, but this one is known to work well [35].

In this approach, the asymptotic behaviour of the elastic cross section is $\sigma_{e l} \propto$ $\sigma_{\text {tot }}^{2} / B_{e l} \propto s^{2 \epsilon} / \ln s$. As $\sigma_{\text {tot }}$ itself, $\sigma_{e l}$ therefore has an unphysically rapid increase. But while the Froissart-Martin bound for $\sigma_{\text {tot }}$ is respected up to energies of around $10^{23} \mathrm{GeV}$, the Pumplin bound $\sigma_{e l} / \sigma_{\text {tot }} \leq 1 / 2[36]$ is violated already at about $10^{6} \mathrm{GeV}$. In order to avoid this, we have made the substitution

$$
2 \alpha^{\prime} \ln \left(\alpha^{\prime} s\right) \approx 0.5 \ln (0.25 s) \longrightarrow 4 s^{\epsilon}-4.2 .
$$

In the energy range $10-100 \mathrm{GeV}$, where the bulk of the data is found, this hardly implies any numerical changes. It is also consistent with $\bar{p} p$ data at higher energies [37]. Asymptotically one obtains a constant ratio $\sigma_{e l} / \sigma_{t o t}$.

\subsection{The VMD diffractive cross sections}

The conversion from $V \mathrm{p}$ to $\gamma \mathrm{p}$ diffractive cross sections is again done routinely, as in eqs. (20) and (28), so this section only deals with $V$ p cross sections.

In a model with a factorized pomeron interaction, the single diffractive cross section $A+B \rightarrow A+X$ is given by

$$
\frac{\mathrm{d}^{2} \sigma_{s d}}{\mathrm{~d} t \mathrm{~d} m_{X}^{2}}=\frac{G_{3 \mathbb{P}}(t)}{16 \pi m_{X}^{2}} \beta_{A \mathbb{P}}^{2}(t) \beta_{B \mathbb{P}}(0)\left(\frac{s}{m_{X}^{2}}\right)^{2\left(\alpha_{\mathbb{T}}(t)-1\right)}\left(m_{X}^{2}\right)^{\alpha_{\mathbb{P}}(0)-1},
$$

where $\beta_{A \mathbb{P}}(t)=\beta_{A \mathbb{P}}(0) \exp \left(b_{A} t\right)=\beta_{A \mathbb{P}} \exp \left(b_{A} t\right)$. Data show that the triple pomeron coupling $G_{3 \mathbb{P}}(t) \approx G_{3 \mathbb{P}}(0) \equiv G_{3 \mathbb{P}}$. One may for instance use the number $2 \sigma_{s d}=4.6 \mathrm{mb}$, determined for $\mathrm{pp}$ interactions at $\sqrt{s}=20 \mathrm{GeV}$, in the region $1.5 \mathrm{GeV}^{2} \approx\left(m_{\mathrm{p}}+2 m_{\pi}\right)^{2} \leq$ $m_{X}^{2} \leq 0.15 s[38]$, to fix $G_{3 \mathbb{P}}$.

The problem is that $\sigma_{s d}$, as defined above and with $\alpha_{\mathbb{P}}(0)-1=\epsilon \approx 0.08$, is growing much faster than $\sigma_{t o t}$ : one obtains $2 \sigma_{s d} / \sigma_{t o t} \approx 1$ already at $40 \mathrm{TeV}$. So once again we encounter the inconsistencies present in a simple pomeron approach with $\epsilon>0$. A change in the energy dependence of the slope, equivalent to the substitution in eq. (30), is here not enough to stave off the divergence.

It is therefore tempting to return to the older pomeron description of cross sections, wherein $\epsilon \equiv 0$, in the study of diffractive processes. If so, one has the following expressions for reactions in eqs. (8)-(10):

$$
\begin{aligned}
\sigma_{t o t}^{A B}(s) & =\beta_{A \mathbb{P}} \beta_{B \mathbb{P}}, \\
\sigma_{s d(A X)}^{A B}(s) & =\frac{G_{3 \mathbb{P}}}{16 \pi} \beta_{A \mathbb{P}}^{2} \beta_{B \mathbb{P}} \iint \frac{\mathrm{d} m_{X}^{2}}{m_{X}^{2}} \mathrm{~d} t F_{s d} e^{B_{s d(A X)} t}=\frac{G_{3 \mathbb{P}}}{16 \pi} \beta_{A \mathbb{P}}^{2} \beta_{B \mathbb{P}} \mathcal{I}_{A X}, \\
\sigma_{s d(X B)}^{A B}(s) & =\frac{G_{3 \mathbb{P}}}{16 \pi} \beta_{A \mathbb{P}} \beta_{B \mathbb{P}}^{2} \iint \frac{\mathrm{d} m_{X}^{2}}{m_{X}^{2}} \mathrm{~d} t F_{s d} e^{B s d(X B)^{t}}=\frac{G_{3 \mathbb{P}}}{16 \pi} \beta_{A \mathbb{P}} \beta_{B \mathbb{P}}^{2} \mathcal{I}_{X B}, \\
\sigma_{d d}^{A B}(s) & =\frac{G_{3 \mathbb{P}}^{2}}{16 \pi} \beta_{A \mathbb{P}} \beta_{B \mathbb{P}} \iiint \frac{\mathrm{d} m_{X 1}^{2}}{m_{X 1}^{2}} \frac{\mathrm{d} m_{X 2}^{2}}{m_{X 2}^{2}} \mathrm{~d} t F_{d d} e^{B_{d d} t}=\frac{G_{3 \mathbb{P}}^{2}}{16 \pi} \beta_{A \mathbb{P}} \beta_{B \mathbb{P}} \mathcal{I}_{X X} .
\end{aligned}
$$


Here $F_{s d}$ and $F_{d d}$ are correction factors (introduced by us) to be detailed below and the slope parameters $B_{i}=B_{i}\left(s, m_{X}^{2}\right)$ are

$$
\begin{aligned}
B_{s d(A X)} & =2 b_{A}+2 \alpha^{\prime} \ln \left(\alpha^{\prime} s m_{\mathrm{p}}^{2} / m_{X}^{2}\right), \\
B_{s d(X B)} & =2 b_{B}+2 \alpha^{\prime} \ln \left(\alpha^{\prime} s m_{\mathrm{p}}^{2} / m_{X}^{2}\right), \\
B_{d d} & =2 \alpha^{\prime} \ln \left(\alpha^{\prime} s m_{\mathrm{p}}^{4} /\left(m_{X 1}^{2} m_{X 2}^{2}\right)\right) .
\end{aligned}
$$

The reggeon term has been neglected, as appropriate for a high-energy description. Further, the reggeon diffractive mass spectrum is not peaked at low masses, and therefore should give a negligible contribution to the experimental diffractive cross section. The reggeon part is more important for elastic scattering, but here we use the optical theorem anyway, i.e. we do not use the pomeron-based formula

$$
\sigma_{e l}^{A B}(s)=\frac{1}{16 \pi} \beta_{A \mathbb{P}}^{2} \beta_{B \mathbb{P}}^{2} \int \mathrm{d} t e^{B_{e l} t}=\frac{1}{16 \pi} \beta_{A \mathbb{P}}^{2} \beta_{B \mathbb{P}}^{2} \mathcal{I}_{e l},
$$

with $B_{e l}$ given by eq. (29), but include it here only for completeness.

The kinematical range for $m_{X}$ in $A+B \rightarrow A+X$ is

$$
m_{B}+2 m_{\pi}<m_{X}<\sqrt{s}-m_{A} .
$$

The lower limit is a good approximation to where the experimental diffractive mass spectrum turns on [38], but clearly a more detailed threshold modelling would be needed to fully describe the low-mass resonance region, i.e. processes such as $p p \rightarrow p \Delta^{+}$, see e.g. ref. [39]. At the other extreme, when $m_{X}^{2} \rightarrow s$, one expects a stronger dampening than provided by the scale-invariant $\mathrm{d} m_{X}^{2} / m_{X}^{2}$ spectrum. Therefore an additional factor $F_{s d}=1-m_{X}^{2} / s$ is inserted into $\mathcal{I}_{A X}$ and $\mathcal{I}_{X B}$. This is more of aesthetical than practical importance, since the experimental region $m_{X}^{2} \approx s$ is dominated by non-diffractive events anyway.

In double diffraction $A+B \rightarrow X_{1}+X_{2}$, the kinematical range is

$$
\begin{aligned}
& m_{A}+2 m_{\pi}<m_{X 1}<\sqrt{s}-m_{B}-2 m_{\pi}, \\
& m_{B}+2 m_{\pi}<m_{X 2}<\sqrt{s}-m_{A}-2 m_{\pi}, \\
& m_{X 1}+m_{X 2}<\sqrt{s} .
\end{aligned}
$$

As for single diffraction, a dampening factor $1-\left(m_{X 1}+m_{X 2}\right)^{2} / s$ is introduced into $\mathcal{I}_{X X}$ to reduce the event rate close to the kinematical limit. However, unlike the single-diffraction case, this factor does not suppress the region of small rapidity gaps: the two systems $X_{1}$ and $X_{2}$ may well have masses much smaller than $\sqrt{s}$ and yet large enough that their decay products overlap in rapidity space. Then the event topology is indistinguishable from that of non-diffractive events, and the standard description of double diffraction breaks down. In particular, $B_{d d}$ may become negative, i.e. the integral over $t$ may blow up. Therefore two modifications are introduced. Firstly, the total rapidity range of the event is $y_{0} \approx \ln \left(s / m_{\mathrm{p}}^{2}\right)$, while that of each diffractive system is $y_{i} \approx \ln \left(m_{X i}^{2} / m_{\mathrm{p}}^{2}\right)$. (The typical transverse mass of produced particles is taken to be $m_{\mathrm{p}} \approx m_{\rho 0}$.) The typical rapidity gap is then $\Delta=y_{0}-y_{1}-y_{2}$, and a suitable suppression factor to remove events with $\Delta<0$ is

$$
\frac{1}{1+e^{-\Delta}}=\frac{e^{y_{0}}}{e^{y_{0}}+e^{y_{1}+y_{2}}}=\frac{s m_{\mathrm{p}}^{2}}{s m_{\mathrm{p}}^{2}+m_{X 1}^{2} m_{X 2}^{2}} .
$$


In total, the correction factor $F_{d d}$ in eq. (35) thus is $F_{d d}=\left[1-\left(m_{X 1}+m_{X 2}\right)^{2} / s\right] /[1+$ $\exp (-\Delta)$ ]. Secondly, an arbitrary term $e^{4}$ is added to the argument of the logarithm of $B_{d d}$,

$$
B_{d d}=2 \alpha^{\prime} \ln \left(e^{4}+\frac{\alpha^{\prime} s m_{\mathrm{p}}^{4}}{m_{X 1}^{2} m_{X 2}^{2}}\right),
$$

to keep $B_{d d}$ from becoming smaller than $8 \alpha^{\prime} \approx 2 \mathrm{GeV}^{-2}$.

The kinematical range in $t$ depends on all the masses of the problem. In terms of the scaled variables $\mu_{1}=m_{A}^{2} / s, \mu_{2}=m_{B}^{2} / s, \mu_{3}=m_{X 1}^{2} / s\left(=m_{A}^{2} / s\right.$ when $A$ scatters elastically), $\mu_{4}=m_{X 2}^{2} / s\left(=m_{B}^{2} / s\right.$ when $B$ scatters elastically $)$, and the combinations

$$
\begin{aligned}
& C_{1}=1-\left(\mu_{1}+\mu_{2}+\mu_{3}+\mu_{4}\right)+\left(\mu_{1}-\mu_{2}\right)\left(\mu_{3}-\mu_{4}\right), \\
& C_{2}=\sqrt{\left(1-\mu_{1}-\mu_{2}\right)^{2}-4 \mu_{1} \mu_{2}} \sqrt{\left(1-\mu_{3}-\mu_{4}\right)^{2}-4 \mu_{3} \mu_{4}}, \\
& C_{3}=\left(\mu_{3}-\mu_{1}\right)\left(\mu_{4}-\mu_{2}\right)+\left(\mu_{1}+\mu_{4}-\mu_{2}-\mu_{3}\right)\left(\mu_{1} \mu_{4}-\mu_{2} \mu_{3}\right),
\end{aligned}
$$

one has $t_{\min }<t<t_{\max }$ with

$$
\begin{aligned}
& t_{\text {min }}=-\frac{s}{2}\left(C_{1}+C_{2}\right), \\
& t_{\text {max }}=-\frac{s}{2}\left(C_{1}-C_{2}\right)=-\frac{s}{2} \frac{4 C_{3}}{C_{1}+C_{2}}=\frac{s^{2} C_{3}}{t_{\text {min }}} .
\end{aligned}
$$

In the limit that all $\mu_{i}$ are small, this simplifies to

$$
\begin{aligned}
& t_{\text {min }} \approx-s, \\
& t_{\text {max }} \approx\left\{\begin{array} { l } 
{ - s ( \mu _ { 3 } - \mu _ { 1 } ) ( \mu _ { 4 } - \mu _ { 2 } ) } \\
{ - s \mu _ { 1 } ( \mu _ { 4 } - \mu _ { 2 } ) ^ { 2 } }
\end{array} \text { for } \quad \left\{\begin{array}{l}
\mu_{3} \neq \mu_{1}, \mu_{4} \neq \mu_{2} \\
\mu_{3}=\mu_{1}, \mu_{4} \neq \mu_{2}
\end{array} .\right.\right.
\end{aligned}
$$

The c.m. frame scattering angle $\theta$ is related to $t$ by $t=-s\left(C_{1}-C_{2} \cos \theta\right) / 2$. When $A$ represents an incoming photon, it is not clear what mass to use for the definition of $\mu_{1}$. We have chosen $m_{A}=m_{V}$ rather than $=0$; in this way VMD elastic scattering has the limit $t_{\max }=0$.

The integrals $\mathcal{I}_{A X}, \mathcal{I}_{X B}$ and $\mathcal{I}_{X X}$, with all kinematical constraints and including the above dampening factors, are too complex to be evaluated analytically. However, analytical results obtained under simplifying conditions provide a good starting point for parametrizing the results of complete numerical evaluations, as follows.

If the $t$ range is assumed to be $-\infty<t<0$ and no dampening factor $1-m_{X}^{2} / s$ is included, one obtains analytically

$$
\mathcal{I}_{A X}=\frac{1}{2 \alpha^{\prime}} \ln \left(\frac{2 b_{A}+2 \alpha^{\prime} \ln \left(\alpha^{\prime} s m_{\mathrm{p}}^{2} / m_{\text {min }}^{2}\right)}{2 b_{A}+2 \alpha^{\prime} \ln \left(\alpha^{\prime} s m_{\mathrm{p}}^{2} / m_{\max }^{2}\right)}\right),
$$

where $m_{\min }$ and $m_{\max }$ are given by eq. (40). Both the restriction of the $t$ range and the dampening factor $F_{s d}$ act to suppress the importance of the large-mass tail. Therefore a good approximation to the numerical result can be obtained by considering $m_{\max }$ as a free parameter,

$$
m_{\text {max }}^{2}= \begin{cases}s\left(0.22+\frac{0.08}{\ln s}\right) & \text { for } A=\mathrm{p}(\text { or } \phi) \\ s\left(0.28+\frac{0.08}{\ln s}\right) & \text { for } A=\rho^{0}(\text { or } \omega)\end{cases}
$$


The integral $\mathcal{I}_{X B}$ is obtained by the trivial substitution $A \leftrightarrow B$. Note that $\mathcal{I}_{X B} \neq \mathcal{I}_{A X}$ for $A \neq B$.

The double diffractive integral $\mathcal{I}_{X X}$ may also be obtained analytically for $-\infty<t<0$, $B_{d d}=2 \alpha^{\prime} \ln \left(m_{\mathrm{p}}^{2} s /\left(m_{X 1}^{2} m_{X 2}^{2}\right)\right)=2 \alpha^{\prime} \Delta, F_{d d}=1$ and $\Delta_{0}<\Delta<y_{0}-y_{\text {min }}$, where $y_{\min }=$ $y_{1 \text { min }}+y_{2 \text { min }}=\ln \left(\left(m_{A}+2 m_{\pi}\right)^{2} / m_{\mathrm{p}}^{2}\right)+\ln \left(\left(m_{B}+2 m_{\pi}\right)^{2} / m_{\mathrm{p}}^{2}\right)$ :

$$
\mathcal{I}_{X X}=\frac{1}{2 \alpha^{\prime}}\left(\left(y_{0}-y_{\text {min }}\right) \ln \left(\frac{y_{0}-y_{\min }}{e \Delta_{0}}\right)+\Delta_{0}\right) \text {. }
$$

The influence of the correct $t$ range, the suppression factors $F_{d d}$ and the true $B_{d d}$ (eq. (45)) may be included by turning $\Delta_{0}$ into an effective parameter,

$$
\Delta_{0}=1.6+\frac{6.5}{\ln s}-\frac{25}{\ln ^{2} s} .
$$

The expression for $\sigma_{\text {tot }}$, eq. (32), is used to define the $\beta_{A \mathbb{P}}$ coefficients: $\beta_{A \mathbb{P}} \beta_{B \mathbb{P}}=$ $X^{A B} s_{0}^{\epsilon}$, where the right-hand side comes from eq. (17). Here $s_{0}$ is the reference scale at which the coefficients $\beta_{A \mathbb{P}}$ and $G_{3 \mathbb{P}}$ are to be determined. As explained above, we have chosen $\sqrt{s_{0}} \approx 20 \mathrm{GeV}$. Then

$$
\begin{aligned}
& \hat{\beta}_{\mathrm{p} \mathbb{P}}=\beta_{\mathrm{pP}} s_{0}^{-\epsilon / 2}=\sqrt{X^{\mathrm{pp}}} \approx 4.658 \mathrm{mb}^{1 / 2}, \\
& \hat{\beta}_{\rho^{0} \mathbb{P}}=\beta_{\rho^{0} \mathbb{P}} s_{0}^{-\epsilon / 2}=\frac{X^{\rho^{0} \mathrm{p}}}{\sqrt{X^{\mathrm{pp}}}} \approx 2.926 \mathrm{mb}^{1 / 2}, \\
& \hat{\beta}_{\phi \mathbb{P}}=\beta_{\phi \mathbb{P}} s_{0}^{-\epsilon / 2}=\frac{X^{\phi \mathrm{p}}}{\sqrt{X^{\mathrm{pp}}}} \approx 2.149 \mathrm{mb}^{1 / 2} .
\end{aligned}
$$

Using $\sigma_{s d}^{\mathrm{pp}}\left(s_{0}\right) \approx 2.3 \mathrm{mb}$, one obtains $G_{3 \mathbb{P}} \approx 0.359 \mathrm{mb}^{1 / 2}$. Reinserting this number into eqs. (33) and (35) and simplifying gives

$$
\begin{aligned}
\sigma_{s d(A X)}^{A B} & =\left(0.0379 \mathrm{mb}^{-1 / 2} \mathrm{GeV}^{-2}\right) X_{A B} \hat{\beta}_{A \mathbb{P}} \mathcal{I}_{A X} \\
\sigma_{d d}^{A B} & =\left(0.0107 \mathrm{GeV}^{-2}\right) X_{A B} \mathcal{I}_{X X} .
\end{aligned}
$$

\subsection{Discussion}

The $\gamma p$ VMD partial cross sections are displayed in Fig. 3. The non-diffractive part is given by what is left of the total VMD cross section when elastic and diffractive contributions have been subtracted, eq. (12). It is important to remember that, since experimental definitions rarely agree with the theoretical ones, the raw partial cross sections do not tell the full story, but have to be seen in the context of differential distributions in diffractive masses and $t$ values.

We have seen how the pomeron picture, which is so successful in describing total cross sections, fails to provide sensible answers when applied to partial ones. As one moves from total to elastic to single to double diffractive, the breakdowns become more severe and appear at lower energies. We have therefore introduced modifications to ensure 'sensible' asymptotic behaviours.

It will therefore be important to test the relevance of our scenario, and this is best done if information from $\gamma p$ and other processes may be combined:

- A comparison of $B_{e l}$ in $\gamma p$ and pp would show whether the ansatz in eq. (29) works, with a $b_{\gamma}$ of the size expected from the VMD ansatz. 
- In the VMD model, the couplings $4 \pi \alpha / f_{V}^{2}$ are supposed to be energy-independent, so it is of the utmost importance to check this assumption. A joint measurement of $\sigma_{e l}$ and $B_{e l}$ for $\gamma+\mathrm{p} \rightarrow V+\mathrm{p}$ implies, via the optical theorem, a measurement of the corresponding total cross section (assuming $\rho \approx 0$ ). Comparing this with the $V+\mathrm{p} \rightarrow V+\mathrm{p}$ total cross section, using e.g. the fits in eqs. (21) and (22), the couplings may be extracted at various energies.

- From a measurement of $\sigma_{s d}$ an effective value for $G_{3 \mathbb{P}}$ may be extracted. If our ansatz is correct, this value should come out to be energy-independent. Since $\bar{p} p / p p$ offers the largest lever arm in energy, this would be the best place for tests.

- Several further checks of our ansatz can be performed, since also the mass spectra and $t$ distributions of diffractive events are predicted.

\section{Jet Cross Sections}

According to our conventions, all direct and anomalous events contain 'hard' jets, and in this sense the cross sections are perturbatively calculable. The VMD class, on the other hand, consists of a mixture of 'hard' and 'soft' events. The hard interactions are of two kinds:

- The direct processes include the Compton graph $\gamma+q \rightarrow q+g$ and the photongluon fusion graph $\gamma+g \rightarrow q+\bar{q}$. The dominant subgraphs involve the exchange of a quark in the $t$ - or $u$-channel, and so the parton-level cross section (at fixed $\hat{s}$ ) roughly behaves like $\mathrm{d} p_{\perp}^{2} / p_{\perp}^{2}$.

- Anomalous and hard VMD events receive contributions from the six graphs $q+q^{\prime} \rightarrow$ $\mathrm{q}+\mathrm{q}^{\prime}, \mathrm{q}+\overline{\mathrm{q}} \rightarrow \mathrm{q}^{\prime}+\overline{\mathrm{q}}^{\prime}, \mathrm{q}+\overline{\mathrm{q}} \rightarrow \mathrm{g}+\mathrm{g}, \mathrm{q}+\mathrm{g} \rightarrow \mathrm{q}+\mathrm{g}, \mathrm{g}+\mathrm{g} \rightarrow \mathrm{g}+\mathrm{g}$ and $\mathrm{g}+\mathrm{g} \rightarrow \mathrm{q}+\overline{\mathrm{q}}$. The bulk of the cross section is associated with the exchange of a gluon in the $t$-channel, and so is of the approximate form $\mathrm{d} p_{\perp}^{2} / p_{\perp}^{4}$.

The hard cross sections may be calculated according to eqs. (13) and (14), given a knowledge of the proton and photon structure functions, and a choice of lower cut-off scales. These aspects will be discussed in turn.

Note that we stay with a leading order description throughout. Thereby a number of additional intricacies are avoided, e.g. the mixing between the direct and anomalous event classes. Effects of higher orders are approximately included by the use of parton showers (see section 6.2).

\subsection{The proton structure function}

A host of proton structure function parametrizations have been produced. For the results presented here, we have been using MRS S0, which is the recommended default of PDFLIB [40]. A few other choices, notably EHLQ 1, have been tried and seen to give similar results, once free parameters have been retuned appropriately.

Existing parametrizations all have a range of validity, however, and we will need to go beyond that. For instance, $f_{a}^{\mathrm{p}}\left(x, \mu^{2}\right)$ of MRS S0 is defined from a $\mu_{0}^{2}=5 \mathrm{GeV}^{2}$ upwards, while direct processes will probe the proton structure function down to $\mu^{2} \approx 0.25 \mathrm{GeV}^{2}$. The corresponding $x$ values at HERA are as small as $x \approx \mu^{2} / s \sim 10^{-5}$, where structure functions have never been measured, and where a lot of uncertainty thus exists.

The behaviour of structure functions is known in two distinct kinematical regions. First in the Bjorken limit $\mu^{2} \rightarrow \infty$ at fixed $x$ (in practice, this is the region $\mu^{2}$ greater than 
about $5 \mathrm{GeV}^{2}$ ). Here perturbative QCD provides a well founded theoretical framework, and structure functions can be expressed in terms of parton distribution functions (PDFs). The second domain where we have (some) information about structure functions is the Regge region, $x \rightarrow 0$ at low $\mu^{2}$, in particular the photoproduction limit $\mu^{2}, x \rightarrow 0$ but $\mu^{2} / x=$ const. Here we can rely only on phenomenology. Since the parton model ceases to be valid at low $\mu^{2}$, it is not clear how to define PDFs as $\mu^{2} \rightarrow 0$. Efforts to combine the knowledge about the two domains have a long history. In fact, the input distributions (at $\mu_{0}^{2}$ ) of most parametrizations of PDFs follow a Regge ansatz and are then evolved towards higher $\mu^{2}$ using perturbative QCD.

An extension of PDFs to values of $\mu^{2}$ much below $\sim 5 \mathrm{GeV}^{2}$ has been tried with the so-called 'radiatively generated' parton distributions [41]. The original hope was that, at some very low resolution scale $\mu^{2}=\mu_{v}^{2} \equiv(2 \Lambda)^{2} \sim 0.2 \mathrm{GeV}^{2}$, the proton consists entirely of valence quarks. Sea quarks and gluons are then radiatively generated through the standard evolution equations of perturbative QCD, postulated to be valid down to $\mu_{v}^{2}$. The agreement with data is not so good, but can be improved if also sea and gluon distributions are allowed at $\mu_{v}^{2}$ [42]. All distributions are still required to have a valence-like form at $\mu_{v}^{2}$, so no ambiguities arise from an arbitrary input behaviour for $x \rightarrow 0$. However, recent deep-inelastic-scattering data [43] indicate important higher-twist contributions at low $\mu^{2}$, questioning the validity of the renormalization group equations down to $\mu_{v}^{2}$. Moreover, as $\mu^{2} \rightarrow 0$, quark distribution functions have to vanish eventually (at least for the dominant vector-current contribution), cf. the electromagnetic structure function $F_{2}^{e m}\left(x, \mu^{2}\right)$, which approaches $\mu^{2}$ times the real $\gamma$ p cross section. By contrast, in the radiative scenario the full momentum of the proton is carried by the partons at all scales $\mu^{2}$, because of the detailed balance of the standard evolution equations. We have therefore not pursued this scenario.

While a description of PDFs valid at arbitrary low $\mu^{2}$ is still lacking, such parametrizations of structure functions do exist, in particular of $F_{2}^{e m}$. Recently, two descriptions were attempted of the electromagnetic structure function $F_{2}^{e m}\left(x, \mu^{2}\right)$ (or, equivalently, of $\left.\sigma^{\gamma^{*} \mathrm{P}}\left(W, \mu^{2}\right)\right)$ at low $\mu^{2}[44,45]$. Both approaches are inspired by Regge theory, but differ in the ansatz of the $\mu^{2} \rightarrow 0$ continuation. For $\mu^{2} \ll \Lambda^{2}$ and $x \ll 1$, however, the structure of their results is similar and predicts for the valence and sea parts, respectively:

$$
\begin{aligned}
& F_{2, \text { sea }}^{e m}\left(x, \mu^{2}\right) \rightarrow c_{\mathbb{P}} x^{-\epsilon} \frac{\mu^{2}}{\mu^{2}+m_{1}^{2}}\left(\frac{\mu^{2}}{\mu^{2}+m_{\mathbb{P}}^{2}}\right)^{\epsilon}, \\
& F_{2, \text { val }}^{e m}\left(x, \mu^{2}\right) \rightarrow c_{\mathbb{R}} x^{\eta} \frac{\mu^{2}}{\mu^{2}+m_{2}^{2}}\left(\frac{\mu^{2}}{\mu^{2}+m_{\mathbb{R}}^{2}}\right)^{-\eta} .
\end{aligned}
$$

In ref. [44] $\epsilon \approx 0.08, \eta \approx 0.56, m_{1}^{2}=m_{\mathbb{P}}^{2} \approx 0.36 \mathrm{GeV}^{2}, m_{2}^{2}=m_{\mathbb{R}}^{2} \approx 0.85 \mathrm{GeV}^{2}, c_{\mathbb{P}} \approx 0.17$ and $c_{\mathbb{R}} \approx 1.33$. In ref. [45] $\epsilon \approx 0.045, \eta \approx 0.60, m_{1}^{2}=m_{2}^{2} \approx 0.30 \mathrm{GeV}^{2}, m_{\mathbb{P}}^{2} \approx 10.7 \mathrm{GeV}^{2}$, $m_{\mathbb{R}}^{2} \approx 0.21 \mathrm{GeV}^{2}, c_{\mathbb{P}} \approx 0.27$ and $c_{\mathbb{R}} \approx 0.68$.

We extend these works in two directions. Firstly, we need parametrizations of the PDFs, including the gluon, not just of $F_{2}^{e m}$. Secondly, we want to cover the complete $\left(x, \mu^{2}\right)$ plane, i.e. (i) include the logarithmic scaling violations valid at large $\mu^{2}$, and (ii) dampen the (potentially) steep increase at small $x$.

To this end we link the small- $x$ behaviours of the valence and sea/gluon distributions to the high-energy behaviour of the reggeon and pomeron parts of the total $\gamma p$ cross section, $\sigma_{t o t}^{\gamma p}=X s^{\epsilon}+Y s^{-\eta}$. At small $x$ and at small $\mu^{2}$ we require the gluon and sea distributions to behave like $x^{-\epsilon}$ and valence distributions to vanish like $x^{\eta}$. The low- $\mu^{2}$ behaviour we 
model by power-like scaling violations proportional to $\left(\mu^{2} /\left(\mu^{2}+m_{\mathbb{R}, \mathbb{P}}^{2}\right)\right)^{\delta_{\mathbb{R}, \mathbb{P}}}$, where the powers $\delta_{\mathbb{R}, \mathbb{P}}$ are fixed by demanding an analytical behaviour in the limit $x, \mu^{2} \rightarrow 0$ at fixed $x / \mu^{2}$. Note that we explicitly violate the momentum sum rule: as $\mu^{2}$ approaches zero, the ability of the photon to resolve the partons within the proton diminishes. The photon rather interacts via its hadronic fluctuations, soft physics, or it couples to the charge of the proton, the Compton process $\gamma p \rightarrow \gamma p$. Furthermore we make the valence distributions harder at low $\mu^{2}$. The normalization and mass parameters $m_{\mathbb{R}, \mathbb{P}}$ we obtain by (i) comparing with the recent small- $x$ data [43] and (ii) requiring that the electromagnetic structure function $F_{2}^{e m}\left(x, \mu^{2}\right)=\sum_{a=\mathrm{q}, \overline{\mathrm{q}}} e_{\mathrm{q}}^{2} x f_{a}^{\mathrm{p}}\left(x, \mu^{2}\right)$ approaches the non-hadron-like part of the total photoabsorption cross section in the limit $x, \mu^{2} \rightarrow 0$ at fixed $x / \mu^{2}$. At large $\mu^{2}$ we match on to given parametrizations of PDFs.

In order to cover the various limits, the $\left(x, \mu^{2}\right)$ plane is divided into four regions, as shown in Fig. 4. The vertical dividing line at $\mu^{2}=\mu_{0}^{2}$, i.e. at about $5 \mathrm{GeV}^{2}$, separates the high- and low- $\mu^{2}$ ranges. In the high- $\mu^{2}$ range, the border curve [46]

$$
\mu_{B}^{2}(x)=2+0.052^{2} \exp \left(3.56 \sqrt{\ln \frac{1 / 3}{x}}\right)
$$

separates regions I and III of large and small $x$. At large $x$, i.e. in region I, the standard QCD evolution equations are valid, and the parametrizations in the literature are applicable without any modification. At the border the partons become densely packed, and the standard evolution equations break down [46, 47]. In the vicinity and beyond the border line (i.e. $x \rightarrow \infty$ at fixed $\mu^{2}$ ), the evolution of structure functions is expected to be dampened but the small- $x$ limit is still an open problem $[16,47]$. On the other hand, it is likely that the behaviour of the structure functions in the photoproduction limit $\left(x, \mu^{2} \rightarrow \infty\right.$ but $\mu^{2} / x=$ const. $)$ holds also for small but fixed $\mu^{2}$. Therefore the low- $x$ behaviour along the line of constant $\mu^{2}=\mu_{0}^{2}$ is assumed to be given by the reggeon and pomeron powers, and for $\mu^{2}>\mu_{0}^{2}$ in region III by interpolation between this behaviour and the standard expression at the border line. At any given large $\mu^{2}$, the steep increase as $x \rightarrow 0$ predicted by the standard evolution equations will hence be flattened out.

For $\mu^{2}<\mu_{0}^{2}$, the structure functions are dampened, so as to vanish in the limit $\mu^{2} \rightarrow 0$. In doing so, we neglect the (small) axial-current contributions, which are finite in the $\mu^{2} \rightarrow 0$ limit. The high- and low- $x$ regions are separated at a constant $x_{0}$ defined by $\mu_{B}\left(x_{0}\right)=\mu_{0}$. Numerically, $x_{0} \approx 0.0069$ if $\mu_{0}^{2}=5 \mathrm{GeV}^{2}$. At low $x$, region IV, the reggeon and pomeron behaviours are again used, combined with the necessity to match the total photoproduction cross section in the limit $x, \mu^{2} \rightarrow 0$. Since we expect valence distributions to become more important at decreasing scales, we dampen them less fast at large $x$ in region II.

The detailed behaviour is given as follows. Denote by $f_{v}$ the valence quark distributions and $f_{s}$ the sea and gluon ones as given in some standard parametrization, and by $\hat{f}_{v}$ and $\hat{f}_{s}$ the corresponding extended quantities we want to calculate. The parameter values below are chosen as $\epsilon=0.08, \eta=0.45, m_{\mathbb{R}}^{2}=0.92 \mathrm{GeV}^{2}$ and $m_{\mathbb{P}}^{2}=0.38 \mathrm{GeV}^{2}$.

Region I: large $\mu^{2}$ and $x$. Here the conventional parametrizations are applicable, i.e.

$$
\hat{f}_{v, s}\left(x, \mu^{2}\right)=f_{v, s}\left(x, \mu^{2}\right) \text {. }
$$

Region II: small $\mu^{2}$, large $x$. Here structure functions are damped and valence distributions 
made harder,

$$
\begin{aligned}
& \hat{f}_{v}\left(x, \mu^{2}\right)=\left(\frac{\mu^{2}}{\mu_{0}^{2}} \frac{\mu_{0}^{2}+m_{\mathbb{R}}^{2}}{\mu^{2}+m_{\mathbb{R}}^{2}}\right)^{(1-\eta)(1-x) /\left(1-x_{0}\right)} f_{v}\left(x, \mu_{0}^{2}\right), \\
& \hat{f}_{s}\left(x, \mu^{2}\right)=\left(\frac{\mu^{2}}{\mu_{0}^{2}} \frac{\mu_{0}^{2}+m_{\mathbb{P}}^{2}}{\mu^{2}+m_{\mathbb{P}}^{2}}\right)^{1+\epsilon} f_{s}\left(x, \mu_{0}^{2}\right) .
\end{aligned}
$$

Region III: large $\mu^{2}$, small $x$. The $\mu$ scale is in the range $\mu_{0}<\mu<\mu_{B}(x)$. An interpolation is performed between the expected behaviours at the extremes of the range,

$$
\begin{aligned}
& x \hat{f}_{v}\left(x, \mu^{2}\right)=N_{1}\left(\frac{x}{x_{0}}\right)^{\eta} x_{0} f_{v}\left(x_{0}, \mu_{0}^{2}\right)+N_{2} x f_{v}\left(x, \mu_{B}^{2}(x)\right), \\
& x \hat{f}_{s}\left(x, \mu^{2}\right)=N_{1}\left(\frac{x}{x_{0}}\right)^{-\epsilon} x_{0} f_{s}\left(x_{0}, \mu_{0}^{2}\right)+N_{2} x f_{s}\left(x, \mu_{B}^{2}(x)\right),
\end{aligned}
$$

where

$$
\begin{aligned}
& N_{1}=\frac{\ln \left(\mu_{B}^{2} / \mu^{2}\right)}{\ln \left(\mu_{B}^{2} / \mu_{0}^{2}\right)}, \\
& N_{2}=1-N_{1}=\frac{\ln \left(\mu^{2} / \mu_{0}^{2}\right)}{\ln \left(\mu_{B}^{2} / \mu_{0}^{2}\right)} .
\end{aligned}
$$

Region IV: small $\mu^{2}$ and $x$. Structure functions are damped in $\mu^{2}$, follow the expected small- $x$ behaviour, and are matched on to the photoproduction cross section

$$
\begin{aligned}
& x \hat{f}_{v}\left(x, \mu^{2}\right)=\left(\frac{x}{x_{0}}\right)^{\eta}\left(\frac{\mu^{2}}{\mu^{2}+m_{\mathbb{R}}^{2}}\right)^{1-\eta}\left\{N_{1}\left(\frac{\mu_{0}^{2}+m_{\mathbb{R}}^{2}}{\mu_{0}^{2}}\right)^{1-\eta} x_{0} f_{v}\left(x_{0}, \mu_{0}^{2}\right)+N_{2} x_{0}^{\eta} N^{v}\right\}, \\
& x \hat{f}_{s}\left(x, \mu^{2}\right)=\left(\frac{x}{x_{0}}\right)^{-\epsilon}\left(\frac{\mu^{2}}{\mu^{2}+m_{\mathbb{P}}^{2}}\right)^{1+\epsilon}\left\{N_{1}\left(\frac{\mu_{0}^{2}+m_{\mathbb{P}}^{2}}{\mu_{0}^{2}}\right)^{1+\epsilon} x_{0} f_{v}\left(x_{0}, \mu_{0}^{2}\right)+N_{2} x_{0}^{-\epsilon} N^{s}\right\},
\end{aligned}
$$

where

$$
\begin{aligned}
& N_{1}=1-\frac{x_{0}-x}{x_{0}} \frac{\mu_{0}^{2}-\mu^{2}}{\mu_{0}^{2}}, \\
& N_{2}=1-N_{1}=\frac{x_{0}-x}{x_{0}} \frac{\mu_{0}^{2}-\mu^{2}}{\mu_{0}^{2}},
\end{aligned}
$$

and the photoproduction matching requirement gives the flavour-dependent coefficients $N_{\mathrm{d}}^{v}=0.121, N_{\mathrm{u}}^{v}=2 N_{\mathrm{d}}^{v}, N_{\mathrm{d}}^{s}=N_{\mathrm{u}}^{s}=N_{\mathrm{d}}^{s}=N_{\overline{\mathrm{u}}}^{s}=0.044, N_{\mathrm{g}}^{s}=5 N_{\mathrm{d}}^{s}$, and $N_{\mathrm{s}}^{s}=N_{\overline{\mathrm{s}}}^{s}=$ $0.5 N_{\mathrm{d}}^{s}$.

The extension defined above ensures that structure functions are continuous across the boundaries between the four regions, but the derivatives are discontinuous. Figure 5 illustrates the modifications introduced for the set we use. Note that our extension can be used with any given PDF parametrization.

\subsection{The photon structure function}

In accordance with our picture of the photon, eq. (3), we decompose the photon distribution functions as

$$
f_{a}^{\gamma}\left(x, \mu^{2}\right)=f_{a}^{\gamma, \text { dir }}\left(x, \mu^{2}\right)+f_{a}^{\gamma, \mathrm{VMD}}\left(x, \mu^{2}\right)+f_{a}^{\gamma, \text { anom }}\left(x, \mu^{2}\right),
$$


where

$$
f_{a}^{\gamma, \operatorname{dir}}\left(x, \mu^{2}\right)=Z_{3} \delta_{a \gamma} \delta(1-x)
$$

By comparison, conventional photon structure functions are defined for resolved processes, i.e. the sum of what we here call hard VMD and anomalous:

$$
f_{a}^{\gamma, \text { res }}\left(x, \mu^{2}\right)=f_{a}^{\gamma, \mathrm{VMD}}\left(x, \mu^{2}\right)+f_{a}^{\gamma, \text { anom }}\left(x, \mu^{2}, p_{0}^{2}\right) .
$$

The evolution equation for the quark structure function is of the form

$$
\frac{\partial f_{\mathrm{q}}^{\gamma, \text { res }}\left(x, \mu^{2}\right)}{\partial \ln \mu^{2}}=\int_{x}^{1} \frac{\mathrm{d} y}{y} f_{\mathrm{q}}^{\gamma, \text { res }}\left(y, \mu^{2}\right) \frac{\alpha_{S}}{2 \pi} P_{\mathrm{q} \rightarrow \mathrm{qg}}\left(\frac{x}{y}\right)+3 e_{\mathrm{q}}^{2} \frac{\alpha}{2 \pi}\left(x^{2}+(1-x)^{2}\right),
$$

with $P_{\mathrm{q} \rightarrow \mathrm{qg}}(z)=(4 / 3)\left(1+z^{2}\right) /(1-z)$. The first term is the one present in standard (i.e. hadronic) evolution equations, e.g. for the proton. It describes the influx of partons $q$ at $x$ by branchings $\mathrm{q} \rightarrow \mathrm{qg}$ of partons at $y>x$. Virtual corrections also give the outflux from $x$ to even smaller momentum fractions. The second term, the so-called anomalous one, comes from branchings $\gamma \rightarrow \mathrm{q} \overline{\mathrm{q}}$, and is unique for the photon evolution equations.

Conventional photon structure function parametrizations are made for $f^{\gamma, \text { res }}$ as a whole, by selecting an ansatz at some convenient small scale $\mu_{0}$ and then evolving from there on with eq. (78) (plus the evolution equation for the gluon, of course). Since also quarks produced by $\gamma \rightarrow \mathrm{q} \overline{\mathrm{q}}$ may undergo subsequent $\mathrm{q} \rightarrow \mathrm{qg}$ branchings, there is no simple subdivision of the final structure functions into two terms, one hadron-like and one anomalous.

We therefore have to provide our own structure functions, which are matched to our needs. The ansatz for $f_{a}^{\gamma, V M D}$ follows eq. (3)

$$
f_{a}^{\gamma, \operatorname{VMD}}\left(x, \mu^{2}\right)=\sum_{V} \frac{4 \pi \alpha}{f_{V}^{2}} f_{a}^{V}\left(x, \mu^{2}\right),
$$

i.e. the VMD part is obtained in the same spirit as the VMD partial cross sections, cf. eq. (20). The $f_{a}^{V}\left(x, \mu^{2}\right)$ are chosen to be $f_{a}^{\rho^{0}}=f_{a}^{\omega}=f_{a}^{\pi^{0}}=\left(f_{a}^{\pi^{+}}+f_{a}^{\pi^{-}}\right) / 2$, while $f_{a}^{\phi}$ has been approximated by $f_{\mathrm{s}, v a l}^{\phi}=f_{\mathrm{u}, v a l}^{\pi^{+}}$and $f_{\text {sea }}^{\phi}=f_{\text {sea }}^{\pi^{+}}$. Any conventional $f_{a}^{\pi^{+}}$ parametrization may therefore simply be turned into an $f_{a}^{\gamma, \text { VMD }}$ one. Remember that the pion distribution functions are obtained as solutions of the homogeneous evolution equations, i.e. the solutions of eq. (78) with the inhomogeneous term proportional to $\alpha$ set to zero. The $f_{a}^{\gamma, \text { anom }}$ part is defined to vanish at $\mu^{2}=p_{0}^{2}$, i.e. $f_{a}^{\gamma, \text { anom }}\left(x, p_{0}^{2}, p_{0}^{2}\right) \equiv 0$. The evolution equation for $\mu^{2}>p_{0}^{2}$ is the same as for $f_{a}^{\gamma, \text { res }}$ in eq. (78). Therefore $f_{a}^{\gamma, \text { anom }}$ is entirely perturbatively calculable. We use the parametrization of Ref. [48].

Note that the VMD structure functions contains as much $u$ as $d$ quarks. Based on charge arguments alone, one would expect four times as much u valence quarks as $\mathrm{d}$ ones (as is obtained in $f_{a}^{\gamma, \text { anom }}$ ). In terms of amplitudes, this corresponds to $e_{\mathbf{u}}|u \bar{u}\rangle+$ $e_{\mathrm{d}}|\mathrm{d} \overline{\mathrm{d}}\rangle \simeq\left|\rho^{0}\right\rangle+(1 / 3)|\omega\rangle$. The relative suppression of $\omega$ is in reasonable agrement with the experimental $f_{V}^{2} / 4 \pi$ couplings used here. However, in the diagonal VMD ansatz the information on the relative phase is assumed wiped out in the transition $\gamma \rightarrow V$. Any effect of the phase might be visible in Drell-Yan or prompt photon cross sections, but hardly in the hadronic cross sections presented in this paper.

Consider now the total momentum fraction carried by partons inside a hadron $A$

$$
\mathcal{I}^{A}\left(\mu^{2}\right)=\sum_{a=\mathrm{q}, \overline{\mathrm{q}}, \mathrm{g}} \int_{0}^{1} x f_{a}^{A}\left(x, \mu^{2}\right) \mathrm{d} x
$$


One has $\mathrm{d} \mathcal{I}^{A} / \mathrm{d}\left(\ln \mu^{2}\right)=0$, since the standard evolution equations obey detailed balance. Furthermore $\mathcal{I}^{A}\left(\mu^{2}\right)=1$, i.e. the total hadron momentum is carried by the constituent partons. (We violate this by our modifications in the previous section, letting $\mathcal{I}^{\mathrm{p}}\left(\mu^{2}\right) \rightarrow 0$ when $\mu^{2} \rightarrow 0$, i.e. we then assume that the proton is only partly resolved. The above equality is assumed to be valid only when $\mu^{2}$ is sufficiently large that perturbation theory can be trusted and higher-twist effects are negligible.) Since we assume that $\mathcal{I}^{A}\left(\mu^{2}\right)=1$ also for $A=V$, we obtain

$$
\mathcal{I}^{\gamma, \mathrm{VMD}}\left(\mu^{2}\right)=\sum_{V} \frac{4 \pi \alpha}{f_{V}^{2}}
$$

In contrast with a hadron, the photon contains also partons and leptons, arising from the splittings $\gamma \rightarrow q \bar{q}$ and $\gamma \rightarrow \ell^{+} \ell^{-}$, respectively. The momentum fraction carried by the partons is governed by the anomalous term and gives

$$
\frac{\mathrm{d} \mathcal{I}^{\gamma, \text { anom }}\left(\mu^{2}\right)}{\mathrm{d}\left(\ln \mu^{2}\right)}=\sum_{\mathrm{q}, \overline{\mathrm{q}}} \int_{0}^{1} x 3 e_{\mathrm{q}}^{2} \frac{\alpha}{2 \pi}\left(x^{2}+(1-x)^{2}\right) \mathrm{d} x=\frac{\alpha}{2 \pi} 2 \sum_{\mathrm{q}} e_{\mathrm{q}}^{2} .
$$

Equation (82) implies that the total momentum carried by these partons increases with $\mu^{2}$ :

$$
\mathcal{I}^{\gamma, \text { anom }}\left(\mu^{2}\right)=\frac{\alpha}{2 \pi} 2 \sum_{\mathbf{q}} e_{\mathbf{q}}^{2} \ln \frac{\mu^{2}}{p_{0}^{2}}
$$

Of course, there must be a corresponding loss of unresolved (direct) photons, i.e. one should use $Z_{3}<1$ in eq. (76), where $Z_{3}$ is calculated from

$$
\begin{aligned}
1=\mathcal{I}^{\gamma}\left(\mu^{2}\right) & =\mathcal{I}^{\gamma, \text { dir }}\left(\mu^{2}\right)+\mathcal{I}^{\gamma, \mathrm{VMD}}\left(\mu^{2}\right)+\mathcal{I}^{\gamma, \text { anom }}\left(\mu^{2}\right)+\mathcal{I}^{\gamma, \text { lepton }}\left(\mu^{2}\right) \\
& =Z_{3}+\sum_{V} \frac{4 \pi \alpha}{f_{V}^{2}}+\frac{\alpha}{2 \pi} 2 \sum_{\mathrm{q}} e_{\mathrm{q}}^{2} \ln \frac{\mu^{2}}{p_{0}^{2}}+\frac{\alpha}{2 \pi} \frac{2}{3} \sum_{\ell} \ln \frac{\mu^{2}}{m_{\ell}^{2}} .
\end{aligned}
$$

In practice $\mathcal{I}^{\gamma, \mathrm{VMD} \text { tanom+lepton }}\left(\mu^{2}\right) \ll 1$ and so it is not really necessary to modify $f_{a}^{\gamma, \text { dir,nä̈ve }}=\delta_{a \gamma} \delta(1-x)$.

The physics picture we have in mind is the following. Fluctuations $\gamma \leftrightarrow q \bar{q}$ below the virtuality scale $p_{0}$ have the time to develop into full vector meson states. The amount of such fluctuations cannot be evaluated perturbatively, but is parametrized by the $4 \pi \alpha / f_{V}^{2}$ couplings. At the scale $p_{0}$, the photon therefore only contains a VMD component. When the probing scale is increased above $p_{0}$, the VMD component remains of constant magnitude, $\mathrm{d} \mathcal{I}^{\gamma, \mathrm{VMD}} / \mathrm{d}\left(\ln \mu^{2}\right)=0$, but undergoes the same standard evolution as an ordinary hadron would. However, above $p_{0}$ perturbative $\gamma \leftrightarrow q \bar{q}$ fluctuations are also possible, and these are included in the anomalous structure function. The amount of fluctuations up to a scale of $\mu$ is given by $\left(e / f_{\bar{q} \bar{q}}\right)^{2}=\mathcal{I}^{\gamma \text {,anom }}\left(\mu^{2}\right)$ (cf. eqs. (3) and (83)). Inside these fluctuations further splittings $q \rightarrow$ qg may occur, again according to standard evolution equations.

In principle, our ansatz for the photon structure function contains little freedom: $4 \pi \alpha / f_{V}^{2}$ and $f_{a}^{V}\left(x, \mu^{2}\right)$ are assumed to be already known, and $f_{a}^{\gamma, a n o m}\left(x, \mu^{2}, p_{0}^{2}\right)$ is fully calculable. Only the choice of $p_{0}$ scale remains, and even that is tuned from other considerations. In practice, the pion distribution functions $f_{a}^{\pi^{+}}\left(x, \mu^{2}\right)$ are rather poorly constrained experimentally (in particular, the relative amount of sea, because both the NLO 
QCD corrections and $\mathrm{K}$-factors are applied when extracting the valence distributions from Drell-Yan data, see e.g. ref. [49]), so some uncertainty still exists. By contrast, the conventional approach allows $f_{a}^{\gamma, r e s}$ to be chosen completely freely, in shape and normalization, at some scale $\mu^{2}=\mu_{0}^{2}$.

It is therefore interesting to check how well our constrained ansatz agrees with conventional photon structure functions. As it turns out, the spread between the different structure functions on the market [40] is large, ours being also in that general band. One example is shown in Fig. 6, where the $f_{a}^{\gamma, \mathrm{VMD}}$ is derived from Owens 1 (very similar to what is obtained for instance with the more recent SMRS-P 2), while the $f_{a}^{\gamma, \text { res }}$ is that of Drees-Grassie. The agreement between VMD+anomalous and resolved is seen to be reasonable, except for the gluon structure function at small $x$. This is one place where the spread is notoriously large, however: the Gordon-Storrow parametrizations here are in much better agreement with us, while the LAC ones are significantly above Drees-Grassie. Measurements in the region of small $x$ will therefore be especially interesting.

A more inclusive measure, still rather revealing, is $\mathcal{I}^{\gamma, \text { res }}\left(\mu^{2}\right)$, i.e. the total momentum fraction carried by partons. This is shown for a number of parametrizations in Fig. 7, in (a) for the full $x$ range (in practice $10^{-5}<x<1$ ) and in (b) for the restricted range $0.2<x<0.8, \mathcal{I}_{[0.2,0.8]}^{\gamma, \text { res }}\left(\mu^{2}\right)$. The latter is of interest, since this is the region where data used in fits has been concentrated, so here parametrizations would be expected to agree better than for the full $x$ range.

A first comment is that the slope of $\mathcal{I}^{\gamma, \text { res }}\left(\mu^{2}\right)$ is uniquely predicted, eq. (82). Above the charm threshold, i.e. assuming four flavours, $\mathrm{d} \mathcal{I}^{\gamma, \text { res }}\left(\mu^{2}\right) / \mathrm{d}\left(\ln \mu^{2}\right)=(\alpha / 2 \pi) 2 \sum e_{\mathrm{q}}^{2}=$ $(20 / 9)(\alpha / 2 \pi)$. Of the sets we have considered (which excludes, in particular, several higher-order fits), the GRV LO one only contains three flavours, and therefore has a smaller slope. The DO set is based on a truncated (asymptotic) version of the evolution equations, and has an unphysically large slope. The rest have the expected slope, within errors. They cluster in two bands:

- The Drees-Grassie and Gordon-Storrow parametrizations, which agree well with our expectations. In particular, with some uncertainty they can be extrapolated back in $\mu^{2}$ to the scale $p_{0}$ at which $\mathcal{I}^{\gamma, \text { res }}\left(p_{0}^{2}\right)=0.004=\mathcal{I}^{\gamma, \mathrm{VMD}}$, and one then obtains $p_{0} \sim 0.5 \mathrm{GeV}$, in general agreement with what we will find from other considerations in the next subsection.

- The three LAC parametrizations, which have much larger $\mathcal{I}^{\gamma, r e s}\left(\mu^{2}\right)$. A backwards extrapolation would need an unphysically small $p_{0}$ to make contact with $\mathcal{I}^{\gamma, \mathrm{VMD}}$, so these structure functions are not compatible with our picture of the photon. We note that in the region $0.2<x<0.8$ two of the three agree with the other sets, while LAC 3 is high also here.

In conclusion, we find that our constrained form of the photon structure function is a viable alternative that should be further tested against data. By its subdivision of VMD and anomalous contributions, it allows a distinction between events that we argue should have different properties.

In principle also the photon structure function should be modified at small $x$ and $\mu^{2}$, at least for the VMD part, the way the proton structure functions were extended in the previous subsection. However, we will not have to make use of photon structure functions below $p_{\perp \text { min }} \approx 1.3 \mathrm{GeV}$, unlike the proton where we need to go to $p_{0} \approx 0.5 \mathrm{GeV}$. The $x$ values will also not be as small. In the end, we have therefore not modified the photon structure functions. 


\subsection{Transverse momentum cut-offs}

The differential interaction rate $\mathrm{d} \sigma_{i n t} / \mathrm{d} p_{\perp}$ can be calculated from eq. (13), using perturbative matrix elements and the structure functions discussed above. Each interaction is assumed to produce two (parton level) jets of opposite and compensating $p_{\perp}$, so $\mathrm{d} \sigma_{j e t} / \mathrm{d} p_{\perp}=2 \mathrm{~d} \sigma_{i n t} / \mathrm{d} p_{\perp}$. At reasonably large $p_{\perp}$, the jet rate is therefore well defined. (Unitarity corrections will be discussed in the next subsection.)

To obtain the total interaction and the jet rate, eq. (14), it is necessary to define a lower cut-off. This cut-off defines how far down in $p_{\perp}$ perturbative parton-parton scatterings are assumed to occur. The scattered partons need not have $p_{\perp}$ large enough to show up as separate high- $p_{\perp}$ jets, but even the rate of soft interactions can have consequences, both for the total cross section and for event shapes. Of course, we do not expect any sharp cut-offs to occur in nature, but only smooth, gradual turn-offs. For simplicity of interpretation, and without any significant loss of physics, sharp cut-offs are still used in the following.

Several different cut-off scales may appear in jet events:

- In the direct processes, Fig. 2a, the hard $p_{\perp}$ scale is associated with the $\gamma q \bar{q}$ vertex. Denote this scale $p_{\perp e}^{\text {dir }}$, where the index $e$ stands for 'electromagnetic'. For a direct process to occur we require $p_{\perp e}^{\text {dir }}>p_{0}$.

- In VMD events, Fig. 2b, the hard-scattering subprocesses are QCD ones, dominantly $t$-channel exchange of gluons. The scale $p_{\perp s}^{\mathrm{VMD}}$, where $s$ stands for 'strong', is required to fulfil $p_{\perp s}^{\mathrm{VMD}}>p_{\perp \min }$.

- Anomalous events, Fig. 2c, have both a $\gamma q \bar{q}$ vertex and a QCD hard scattering. One should therefore require both $p_{\perp e}^{\text {anom }}>p_{0}$ and $p_{\perp s}^{\text {anom }}>p_{\perp \text { min }}$. Additionally, it is natural to require $p_{\perp s}^{a n o m}>p_{\perp e}^{a n o m}$. If this latter condition is not fulfilled, the process should be viewed as a direct one, with initial-state-radiation corrections to the incoming parton, as already taken into account by the evolution equations of the proton structure functions.

The choice of $p_{0}=0.5 \mathrm{GeV}$ is based on three arguments, whereof the first two are qualitative and the third one quantitative:

1. If $p_{\perp e}<p_{0}$ in an imagined direct or anomalous process, the $q \bar{q}$ pair at the $\gamma \leftrightarrow q \bar{q}$ vertex has the time to develop into a vector meson. Such an event should then appear in the VMD class. Since we include vector mesons up to a scale of about $m \approx 1 \mathrm{GeV}$ in the VMD description, one should have $p_{0} \sim m / 2 \approx 0.5 \mathrm{GeV}$.

2. If the photon structure function is split into one VMD part and one anomalous one, as is done in the previous subsection, then several of the photon structure function parametrizations available are consistent with a vanishing of the anomalous part at $\mu=p_{0} \sim 0.5 \mathrm{GeV}$.

3. One of the basic assumptions of our model is that the total cross section can be split into direct, VMD and anomalous terms, eq. (6). At low energies, e.g. at $\sqrt{s_{0}} \approx 10 \mathrm{GeV}, \sigma_{\text {anom }}^{\gamma \mathrm{p}}\left(s_{0}\right)$ is negligible, at least in our standard scenario, as we shall soon see. Therefore $\sigma_{d i r}^{\gamma \mathrm{p}}\left(s_{0}\right)$ may be found from a knowledge of $\sigma_{\text {tot }}^{\gamma \mathrm{p}}\left(s_{0}\right)$ and $\sigma_{\mathrm{VMD}}^{\gamma \mathrm{p}}\left(s_{0}\right)$, eqs. $(19)$ and $(23): \sigma_{\text {dir }}^{\gamma \mathrm{p}}\left(s_{0}\right) \approx 114-92=22 \mu \mathrm{b}$. To obtain this interaction rate, a $p_{0} \approx 0.5 \mathrm{GeV}$ is required. This is with our low- $x$ and $-\mu^{2}$ extension of structure functions, applied to MRS S0, and a first-order $\alpha_{S}$ constrained to be $\alpha_{S}<1$.

The choice of $p_{\perp \text { min }}$ scale for QCD interactions has already been discussed in section 3.2 , and we stay with the expression in eq. (15), i.e. basically $p_{\perp \text { min }} \approx 1.3 \mathrm{GeV}$ at HERA 
energies, with the possibility of a slow logarithmic variation. The necessity for a cut-off is motivated by the colour neutrality of the incoming hadrons: an exchanged low- $p_{\perp}$ gluon does not resolve the individual colour charges and therefore its effective coupling vanishes in the limit $p_{\perp} \rightarrow 0$. The actual number is determined from $\bar{p} p$ phenomenology.

\subsection{Jet cross sections}

With the above cut-offs, the integrated hard-interaction rate can be obtained, Fig. 8 . The VMD and the anomalous cross sections are seen to increase rapidly with c.m. energy, a behaviour familiar enough from hadron physics. The direct interaction rate stays fairly constant, and even decreases a bit in the energy range 20-100 GeV. This latter behaviour may be unexpected, and deserves a comment. In direct processes the full photon energy takes part in the hard interaction, i.e. $x_{1}=x_{\gamma}=1$. The hard matrix elements increase as $p_{\perp} \rightarrow 0$ and thus peak at the lower cut-off scale $p_{0}$. Therefore the proton structure function is mainly probed at $x_{2}=x_{\mathrm{p}} \approx p_{0}^{2} / \mathrm{s}$. The energy variation of the direct cross section then closely traces the variation of the structure function $x_{\mathrm{p}}^{0} f_{a}^{\mathrm{p}}\left(x_{\mathrm{p}}^{0}, p_{0}^{2}\right)$ with $x_{\mathrm{p}}^{0}=p_{0}^{2} / s$. Results therefore depend on the assumed small- $x$ behaviour for a fixed small $\mu^{2}$, i.e. on our extension of standard proton structure function parametrizations to this region, cf. Fig. 5. Had we used the standard MRS S0 frozen at $\mu_{0}^{2}=5 \mathrm{GeV}^{2}$, the direct event rate would have been slowly increasing with energy. The $p_{0}$ scale would not have come out very different, $p_{0} \approx 0.6 \mathrm{GeV}$ instead of $0.5 \mathrm{GeV}$. A structure function with a steep increase at small $x$, such as $x f_{a}^{\mathrm{p}}(x) \sim x^{-0.5}$, would have implied a disastrously steep increase of event rate with energy, but fortunately recent NMC data disfavour such a behaviour [43].

The fast increase of VMD and anomalous interaction rates implies that, from around $\sqrt{s} \approx 130 \mathrm{GeV}$, the total interaction rate exceeds the assumed total cross section. This is not allowed, so some new aspect of physics should enter. One possibility would have been if the implied amount of $\gamma \leftrightarrow q \bar{q}$ fluctuations were to become large, i.e. if $\mathcal{I}^{\gamma, \text { res }}\left(\mu^{2}\right)=$ $\mathcal{I}^{\gamma, \text { VMD }}\left(\mu^{2}\right)+\mathcal{I}^{\gamma, \text { anom }}\left(\mu^{2}\right)$ were to be a significant fraction of unity, or, equivalently, if $Z_{3} \ll 1$ (eq. (84)). Then further fluctuations would have been suppressed by unitarity: a photon cannot fluctuate, once more, by $\gamma \rightarrow q \bar{q}$, at a time when it is already in a $q \bar{q}$ state. Direct, VMD and anomalous cross sections would then all be reduced by unitarization effects, and the three event classes would possibly become less distinct. However, as we have already seen, $Z_{3} \approx 1$ for all scales $\mu^{2}$ of interest. There is then no reason to expect any large unitarization effects of the kind that would involve cross-talk between the event classes. We therefore have to seek understanding class by class.

The direct event class is integrated down to very small $p_{\perp}$ values, $p_{\perp}=\mathcal{O}\left(p_{0}\right)$. Hence the cross section is sensitive to the small- $x$ behaviour of the proton $\operatorname{PDFs} f_{a}^{\mathrm{P}}\left(x, \mu^{2}\right)$ at those low $\mu^{2} \sim p_{\perp}^{2}$. As mentioned above, standard parametrizations frozen at $\mu_{0}^{2} \approx 5 \mathrm{GeV}^{2}$ result in 'reasonable' cross sections, provided the input distribution functions $f_{a}^{\mathrm{p}}\left(x, \mu_{0}^{2}\right)$ are flat. Here reasonable means that the direct component stays with increasing energy at or below the $20 \%$ level that we demand at low energy. Our extension of the distribution functions of the proton guarantees such a behaviour for any given parametrization. So we do not consider the case of an exploding direct cross section as would arise from steep PDFs.

VMD cross sections are hadronic ones, and there we know that the naive minijet cross sections explode. For this class, unitarization is achieved as outlined in section 3.2: in hadron-hadron collisions each hadron contains a multitude of partons, and so an event may well contain several interactions. The VMD high- $p_{\perp}$ interactions form part of the 
VMD non-diffractive event class, where they are unitarized together with VMD low- $p_{\perp}$ events. The parametrizations of $\sigma_{\mathrm{VMD}}^{\gamma \mathrm{p}}(s)$ and $\sigma_{n d}^{\gamma \mathrm{p}}(s)$ that we use already implicitly include the effects of all required unitarization, to the best of our current knowledge of $\bar{p} p$ physics.

The problem of the interaction rate exceeding the assumed total $\gamma p$ cross section is once again illustrated in Fig. 9, where the VMD jet rates have been unitarized within the envelope given by $\sigma_{\mathrm{VMD}}^{\mathrm{\gamma}}(s)$. On top of that comes the direct and anomalous contributions. If added at face value, this would amount to about $\sigma_{\text {tot }}^{\gamma \mathrm{p}}(\sqrt{s}=200) \approx 210 \mu \mathrm{b}$, where the predicted and observed number is $\approx 160 \mu \mathrm{b}$. It is thus the remaining difference of $50 \mu \mathrm{b}$ that needs to be 'explained away'. For our main scenario, we will assume that the excess has to be cut out from $\sigma_{\text {anom }}^{\gamma \mathrm{p}}$, although later we will consider alternatives.

Could unitarization effects be at play, so that one single anomalous event contains several interactions, as for VMD? Some such effects should be expected, but we doubt that they are large. The reason is that the anomalous class of $\gamma \leftrightarrow q \bar{q}$ fluctuations form sparse systems of partons - they do not have the time to develop clouds of soft gluons. And it is the large number of such soft gluons that is at the origin of the potentially large rate of multiple interactions in hadron-hadron collisions. One speculative possibility would be to have multiple interactions in those (rare?) events where a small anomalous state hits a 'hot spot' $[50,16]$ in the proton, so that there is an enhanced local density of incoming partons.

So instead we propose another possible explanation. When we motivated the $p_{\perp \text { min }} \approx$ $1.3 \mathrm{GeV}$ cut-off for hadron-hadron collisions, it was related to the typical (colour correlation) size of hadrons. An anomalous $\gamma \leftrightarrow q \bar{q}$ fluctuation is more short-lived than a VMD one, and should also have a smaller spatial extent. This would translate into a requirement of a larger $p_{\perp \text { min }}$ cut-off for anomalous events, i.e. $p_{\perp \text { min }}^{\text {anom }}>p_{\perp \text { min }}^{\mathrm{VMD}}$. At $200 \mathrm{GeV}$, one would require a $p_{\perp \text { min }}^{\text {anom }} \approx 2.2 \mathrm{GeV}$ in order to arrive at the desired $\sigma_{\text {tot }}^{\gamma \mathrm{p}}$. The variation over the energy range of interest, $10 \mathrm{GeV}<\sqrt{s}<300 \mathrm{GeV}$, is reasonably well described by the ansatz

$$
p_{\perp s}^{\text {anom }}>p_{\perp \text { min }}^{\text {anom }}(s)=1.5+0.0035 \sqrt{s}
$$

see Fig. 10, although this particular form of the energy dependence should not be taken too seriously.

The anomalous event rate could also be suppressed in other ways, stemming from the same basic viewpoint. For instance, instead of requiring $p_{\perp s}^{\text {anom }}>p_{\perp e}^{\text {anom }}$, one could impose $p_{\perp s}^{\text {anom }}>r p_{\perp e}^{\text {anom }}$, e.g. with $r \approx p_{\perp m i n}^{\mathrm{VMD}}(s) / p_{0} \approx 2.5$. This translates into the choice of structure function arguments $f_{a}^{\gamma, \text { anom }}\left(x, p_{\perp s}^{2} / r^{2}, p_{0}^{2}\right)$, which gives a sensible cross section over the above energy range. With this recipe, the anomalous jet rate is reduced at all $p_{\perp}$ scales, even if the reduction is largest at small $p_{\perp}$.

\section{The Models}

\subsection{The photon scenarios}

The existence of two photon components (VMD and direct) is well established experimentally, a third one predicted theoretically. Hence we imagine the physical photon as being a superposition of three states, eq. (3). This description is only specified once we fix their relative proportions and prescribe their interactions with the target (the proton, in our case). In the previous sections, we have described the interactions of the various photon 
states with the proton, introduced the relevant scales, and estimated the relative weights. Our experimental inputs are the total $\gamma p$ cross section at low energy, its value at high energy (from a parametrization), data on diffractive production $(\gamma \mathrm{p} \rightarrow V \mathrm{p}$ and $h \mathrm{p} \rightarrow X \mathrm{p})$ and on $\sigma_{\text {had }}^{\mathrm{e}^{+}}$at low energies, and $\mathrm{p}$ and $\pi$ PDF parametrizations. From this information we have argued what we consider as a 'best estimate'. It is still useful (even necessary) to consider some potential alternatives. In total we present five models, whereof model I is our standard scenario and II-V alternative ones. Some of the latter should be easy to discard by comparison with data, while other may help sharpen the experimental tests.

- Model I: 'standard'. This is the standard scenario already discussed. The VMD couplings $4 \pi \alpha / f_{V}^{2}$ are energy-independent. The VMD and direct cross sections are then given, and the anomalous event rate has to be adjusted to fill up the total cross section. This is done by a tuning of $p_{\perp \text { min }}^{\text {anom }}(s)$, the lower $p_{\perp}$ cut-off of anomalous processes, at any given c.m. energy, eq. (85).

- Model II: 'extreme anomalous'. If one wants to use one single cut-off for jet cross sections, namely the one found in hadronic collisions, $p_{\perp m i n}^{a n o m}(s)=p_{\perp m i n}^{\mathrm{VMD}}(s)$, then a large rate of anomalous events is obtained. This could only be accommodated within the total cross section if the VMD component is reduced to make space the direct component is too small to make the difference. One is thus lead to a scenario with $s$-dependent VMD couplings $4 \pi \alpha / f_{V}^{2}$. All the properties of the VMD class remain - the $\rho^{0} / \omega / \phi$ fractions, the decomposition into subclasses, and the properties of events (including multiple interactions) - it is only the rate that is reduced. We include this scenario as an extreme in the direction of a photon with minimal hadron-like component. Ultimately the anomalous cross section will exceed the total one, and then this scenario must break down (unless one finds large unitarity corrections for anomalous events).

- Model III: 'unitarized'. In this scenario, no distinction is made between hard VMD and anomalous events. Instead a conventional photon structure function parametrization is used to calculate a total jet rate, and this rate is unitarized with VMD soft processes according to the standard multiple interactions scenario. There is only one $p_{\perp \text { min }}$ scale, namely the smaller $p_{\perp \text { min }}^{\mathrm{VMD}}$ one, so the scenario contains the maximal amount of jet production. Compared with the standard scenario, there are therefore more multiple interactions, i.e. minimum bias events contain more activity than equivalent hadron-hadron ones. The direct cross section is kept unchanged, and the remaining part of the cross section is made up by the unitarized VMD+anomalous events. The VMD couplings lose their traditional sense in this picture, and play no direct rôle for the jet rate. They still have to be used to define the elastic and diffractive event classes; with some moderate amount of ambiguity, we decided to scale up these by the same amount as the whole VMD+unitarized cross section is scaled up compared with the naïve VMD one. This model is theoretically unsatisfying, because it lacks an underlying picture of the photon and because there is no clear motivation for multiple interactions in anomalous events. Since the treatment of unitarity corrections to anomalous events is not known, however, we include this model as the alternative with maximal jet cross rates.

- Model IV: 'no anomalous'. In this model, it is assumed that the photon has no anomalous component at all, i.e. that there are only direct and VMD events. The VMD couplings have to be made somewhat $s$-dependent, such that the correct total cross section is obtained. By the absence of anomalous events, the photon 
has become more like a vector meson. Note that the anomalous component of the photon has not yet been observed experimentally. A model excluding an anomalous part also does not contradict data on the photon structure function, because of the limited $Q^{2}$-range of two-photon experiments where $F_{2}^{\gamma}\left(x, Q^{2}\right)$ is extracted. Of course, theoretically, we expect the anomalous component, and include this model mainly to help quantify how important the anomalous interactions are.

- Model V: 'pure VMD'. Here the philosophy of model IV is taken one step further: both direct and anomalous events are absent. Everything is thus described in terms of VMD events, with the VMD couplings appropriately scaled up and slightly energy-dependent. Here $\gamma$ p events are no different from $\rho^{0} \mathrm{p}$ ones. Of course, the direct component of the photon has been observed in two-photon physics. But its evidence in (low-energy) photoproduction is very weak so far, seen only at highest $p_{\perp}$. Note also that the pure VMD model does contain partonic constituents and hence gives rise to parton-parton scatterings. It is therefore not necessarily in contradiction with the observation of jets in two-photon physics at AMY.

- Model VI: 'everything else'. This one is here to remind you that the list of possibilities is by no means complete. Here are two examples, which we have not studied in detail:

1. 'Large $\sigma_{\text {tot }}^{\gamma \mathrm{p}}$. All the above models were constrained to add up to the same total $\gamma p$ cross section. However, there is no guarantee that $\sigma_{\text {tot }}^{\gamma \mathrm{p}}(s)$ has to follow the same kind of regularity as observed in hadron-hadron collisions and used as ansatz in eq. (19). The extreme value of $\sigma_{\text {tot }}^{\gamma \mathrm{p}}=210 \mu \mathrm{b}$ at $\sqrt{s}=200 \mathrm{GeV}$ (for $p_{\perp \text { min }}^{\text {anom }}=p_{\perp \text { min }}^{\mathrm{VMD}}=1.3 \mathrm{GeV}$ ) is almost certainly already excluded by HERA, but a value of 170 or $180 \mu \mathrm{b}$ is not, given the large systematical experimental errors that exist. Such cross sections could easily be accommodated in modified versions of model I. A $\sigma_{\text {tot }}^{\gamma \mathrm{p}}<160 \mu \mathrm{b}$ or $>210 \mu \mathrm{b}$ would be less easy to understand.

2. 'Small direct'. In models I-IV above, the direct component is supposed to yield the missing $20 \%$ between the (low-mass) VMD cross section and the observed $\gamma p$ cross section. In the spirit of GVD we can assume that this contribution is rather given by the high-mass hadronic fluctuations of the photon, while the direct component is small. Since we anyhow assume perturbative QCD to be valid down to $p_{0}$, we might as well lower the cut-off for the $2 \rightarrow 2$ scatterings due to the anomalous photon component, i.e. take $p_{\perp}^{\text {anom }}=p_{0}$. At $\sqrt{s}=10 \mathrm{GeV}$ we then obtain an anomalous cross section of about $12 \mu \mathrm{b}$. Thus there is still the need for a non-negligible direct contribution to the total $\gamma p$ cross section. Of course, as energy increases the anomalous cross section quickly exceeds the total cross section $\left(\sigma_{\text {anom }}^{\gamma \mathrm{p}} \approx 200 \mu \mathrm{b}\right.$ at $\left.\sqrt{s}=200 \mathrm{GeV}\right)$, so the cut-off $p_{\perp \text { min }}^{\text {anom }}$ has to be made energy-dependent (alternatively, unitarity corrections have to be applied).

The models are illustrated graphically in Fig. 11.

\subsection{From partons to events}

In the preceding sections we have mainly discussed the behaviour of integrated cross sections. The differential cross-section formulae are displayed, however, so implicitly it should be clear how variables are to be distributed in the full multidimensional phase 
space. But this only gives a description of the parton-level processes. In order to arrive at complete events, it is necessary to add parton showers, beam remnants, fragmentation and decays. This has been performed with the help of the PYTHIA $5.6[22,23]$ and JETSET $7.3[51,23]$ event-generator programs. It would carry us too far to describe the physics aspects simulated in those programs. In the following we will therefore only try to give the flavour of it. Several new aspects were added to Pythia during the course of the current work. Most are directly related to the discussions of the previous sections, while a few others are only mentioned below.

Not all event classes can be generated simultaneously. It it therefore necessary to decide whether to generate direct, VMD or anomalous events in a run - a fourth category also exists for unitarized VMD+anomalous events, as needed in model III. Further, the $\gamma p$ energy is assumed to be fixed. (Recently the possibility of varying energies has been introduced.) The cross section of the class is evaluated by the program, for VMD events by virtue of the $4 \pi \alpha / f_{V}^{2}$ couplings and the parametrized $\sigma_{\text {tot }}^{V \mathrm{p}}$ cross sections, for direct and anomalous by Monte Carlo integration of the interaction cross sections within cuts $p_{0}$ and $p_{\perp \text { min }}$, during the course of the run. These event classes are only afterwards mixed in the appropriate proportions to make up each one of the five models presented above.

The direct and anomalous event classes are homogeneous, but for the VMD class two additional specifications must be made. Firstly, whether the vector meson $V$ is $\rho^{0}, \omega$ or $\phi$, in proportions given by eq. (20). Secondly, whether the event is elastic, single diffractive $(V X$ or $X \mathrm{p})$, double diffractive or non-diffractive, according to the partial cross sections discussed in section 4.

For elastic events, it is only necessary to select the $t$ variable to specify the hard process. The outgoing vector meson is polarized. The $\rho^{0} \rightarrow \pi^{+} \pi^{-}$decay is therefore performed with a $\sin ^{2} \theta$ angular distribution (with respect to the direction of motion of the $\rho^{0}$ in the c.m. frame). This distribution is also used in the $\rho^{0} X$ single diffractive final states. Currently $\omega$ and $\phi$ are allowed to decay isotropically.

In single and double diffraction, the variables to be selected are $t$ and the masses of the diffractive systems. All necessary formulae, including kinematical limits, are given in section 4.4.

A light diffractive system, with a mass less than $1 \mathrm{GeV}$ above the mass of the incoming particle (the $V$ mass for a $\gamma$ ), is taken to decay isotropically into a two-body state. Singleresonance diffractive states, such as a $\Delta^{+}$, are therefore not explicitly generated, but are assumed to be described in an average, smeared-out sense.

A more massive diffractive system is subsequently treated as a string with the quantum numbers of the original hadron. Since the exact nature of the Pomeron exchanged between the hadrons is unknown, two alternatives are available. In the first, the Pomeron is assumed to couple to (valence) quarks, so that the string is stretched directly between the struck quark and the remnant diquark (antiquark) of the diffractive state. In the second, the interaction is rather with a gluon, giving rise to a 'hairpin' configuration in which the string is stretched from a quark to a gluon and then back to a diquark (antiquark). In the current studies we have used the latter alternative, which gives a somewhat larger multiplicity.

There is experimental support for more complicated scenarios [52], wherein the pomeron has a partonic substructure, which e.g. can lead to high- $p_{\perp}$ jet production in the diffractive system. We have not considered such a scenario; the mass of the typical diffractive system is anyway sufficiently low that such activity should not significantly affect average event shapes. 
In non-diffractive events, a sequence of interactions is generated in order of decreasing $p_{\perp}$, according to the differential $p_{\perp}$ distribution in eq. (16). A probability distribution in the number of interactions is automatically obtained. Events with no interactions become low- $p_{\perp}$ ones. Technically they are handled as if originating from the exchange of a zero-momentum gluon between the two incoming hadrons. The two outgoing hadron 'remnants' therefore are colour octets, i.e. they are connected by two colour triplet strings. Each remnant is subdivided into one colour triplet and one antitriplet, e.g. $p=u+u d_{0}$, $\rho^{0}=\mathrm{d}+\overline{\mathrm{d}}$, which share the beam momentum according to some probability distribution, and which are given some relative $p_{\perp}$ smearing of a few hundred $\mathrm{MeV}$, as for the primordial $k_{\perp}$. Each of the two strings in the event is stretched between a triplet in one remnant and an antitriplet in the other. The two strings fragment independently of each other.

When a hard interaction occurs, the two outgoing partons may develop final-state showers, i.e. initiate cascades of branchings $q \rightarrow q+g, g \rightarrow g+g, g \rightarrow q+\bar{q}$ and, optionally, $q \rightarrow q+\gamma$. Initial-state showers are also included, whereby the partons entering the hard interaction may be the end products of showers initiated in the incoming hadrons. The showers smear the kinematics of the naive hard process, and add partonic activity to the final state. Showers may be viewed as a first approximation to the influence of higherorder matrix elements. Some further smearing is obtained by assigning a primordial $k_{\perp}$ to the initial-state shower initiators, with the recoil taken by the beam remnants. The colour flow is traced in the hard interaction and in each step of the parton showers. All the outgoing partons, from hard interaction, initial- and final-state showers, and beam remnants, can thus be connected into a varying number of strings. Each string has a quark, antiquark or diquark at the two ends, plus a varying number of gluons as energy carrying kinks on the string in between.

If several hard interactions occur in an event, the hardest is treated as above. The softer are handled with some simplifications, because of technical reasons and limitations in our physics understanding. Thus no showers are included, and the colour flow is simplified. The main effect of multiple interactions is to increase the event activity at central rapidities, while the energy of the beam remnants is reduced correspondingly.

In direct events, the full photon energy is deposited in the hard interaction, and so there is no photon beam remnant. The parton level thus consists of two hard-scattered partons and one proton beam remnant. The two outgoing partons may develop finalstate showers, while initial-state shower evolution is only possible on the proton side. Primordial $k_{\perp}$ is included for the remnant. The colour flow of the process is traced to give a varying number of strings, which connect the outgoing partons, including the proton beam remnant.

In anomalous events, the QCD $2 \rightarrow 2$ hard interaction is first selected. The outgoing partons may shower, as before. The initial-state shower on the proton side is also standard. On the photon side, one is required to trace the initial-state shower from the QCD hard interaction at scale $p_{\perp s}$ back to the initial $\gamma \rightarrow q \bar{q}$ branching at scale $p_{\perp e}$. This latter scale is chosen logarithmically uniformly in the range $\ln p_{0}<\ln p_{\perp e}<\ln p_{\perp s}$. With the lower cut-off known beforehand, also the photon side initial-state shower may be generated by the standard algorithm. It should be noted that our logarithmically even choice of $p_{\perp e}$ is a simplification - it is reasonable in an average sense, but in a complete picture one would obtain some correlation between the $x$ at the hard interaction and the $p_{\perp e}$; this is too small to worry about here. In the $\gamma \rightarrow \mathrm{q} \bar{q}$ branching, if the $\mathrm{q}$ is the one going in towards the hard interaction, then $\bar{q}$ acts as a kind of beam remnant, which takes the remaining energy of the $\gamma$. The $q$ and $\bar{q}$ are given (oppositely directed) transverse-momentum kicks $p_{\perp e}$, 
which usually are larger than the primordial $k_{\perp}$ otherwise encountered. The $\bar{q}$ remnant could also be allowed to develop a final-state shower, from the scale $p_{\perp e}$ downwards. This has not yet been included, but since $p_{\perp e}$ normally is not all that large we do not expect this to be a serious shortcoming. The colour flow of the full process is traced and used to connect up strings, which then fragment.

Many of the immediate string fragmentation products are unstable, such as $\pi^{0}, \rho, \mathrm{K}^{*}$, $\eta, \Lambda$, D, etc. All such particles are allowed to undergo the required decays. The final state therefore consists of those particles that a good detector can be expected to observe, without making use of any specific vertex reconstruction methods.

\section{Predictions}

Since our model is available in the PYTHIA/JETSET event generator, a multitude of experimental quantities of interest may be predicted or compared with data. Program users can also interface the generated events to detector simulation routines for a detailed understanding of what should be observed in a given detector. In this paper we will therefore only give model results for a few measures, picked to illustrate the main features of the event classes and the five models presented above.

All results are for HERA energies, $\sqrt{s}=200 \mathrm{GeV}$ for the $\gamma p$ system. Events are studied in their c.m. frame. In accordance with HERA conventions, the incoming $\gamma$ is moving in the $-z$ direction and the incoming $\mathrm{p}$ in the $+z$ one.

\subsection{Inclusive events}

We begin by considering the inclusive sample of events. For each figure two frames are given. The top one shows the event properties of four different groups separately, (i) VMD elastic and diffractive, (ii) VMD non-diffractive, (iii) direct and (iv) anomalous. The bottom one compares the five models of section 6.1 , i.e. basically different mixtures of the components above. Note that the top figure is not complete: the class of anomalous events with a low $p_{\perp \text { min }}$ cut-off (in model II) and the VMD+unitarized class (in model III) have been omitted so as not to clutter the figures too much. In both frames, normalization is per event.

Figure 12 shows the 'hard' $p_{\perp}$ scale of the processes - an unobservable quantity, but useful as a reminder of the basic character of processes. Elastic and diffractive events are distributed according to $\exp (B t) \mathrm{d} t \sim p_{\perp} \exp \left(-B p_{\perp}^{2}\right) \mathrm{d} p_{\perp}$. VMD non-diffractive events contain low- $p_{\perp}$ ones at $p_{\perp}=0$ and high- $p_{\perp}$ ones above $p_{\perp \text { min }}^{\mathrm{VMD}}=1.3 \mathrm{GeV}$. The direct and anomalous processes are hard only, beginning at $p_{0}=0.5 \mathrm{GeV}$ and $p_{\perp \min }^{\text {anom }}=2.2 \mathrm{GeV}$, respectively.

The total transverse energy of events, Fig. 13, and the charged multiplicity distribution, Fig. 14, are seen to be very closely related to each other. In a large part, this is because all particles acquire a typical $p_{\perp}$ already as part of the fragmentation process, so that high multiplicities automatically imply large $\sum E_{\perp}$. Thus direct events, which lack a photon beam remnant, have rather low $n_{c h}$ and $\sum E_{\perp}$. And elastic events, with their very small multiplicities, are bound to show up as a peak close to $\sum E_{\perp}=0$. There is also some correlation with the $p_{\perp \text { hard }}$ scale of Fig. 12. For instance, the larger $p_{\perp \text { hard }}$ of anomalous events implies a larger $\sum E_{\perp}$, but also more particles produced in the fragmentation. The VMD non-diffractive events also receive a contribution from multiple interactions - 
most likely this is why the tail of this class to large $\sum E_{\perp}$ and $n_{c h}$ compares well with the anomalous one, although the latter has a larger $p_{\perp \text { hard }}$ scale.

Model II, with its large anomalous component, has a reduced number of VMD elastic+diffractive events, i.e. reduced low- $\sum E_{\perp}$ and $-n_{c h}$ tails, and therefore generally narrower distributions. Models IV and $V$ are the opposite, with more VMD low- $\sum E_{\perp}$ events, although differences compared with the standard are modest. Model III, with VMD+anomalous unitarized, has the largest amount of multiple interactions, and this shows up in the enhanced high- $\sum E_{\perp}$ and $-n_{c h}$ tails.

The rapidity distributions of transverse energy and of charged multiplicity share most features, for reasons already noted, so we only show the former, Fig. 15. The general level of activity has already been discussed, so the interesting aspect is how the photon $(y<0)$ and proton $(y>0)$ hemispheres of the event differ. The asymmetry is largest in the direct class, where the full photon longitudinal momentum is taken by the two high- $p_{\perp}$ jets. The typical $p_{\perp}$ is not really all that high, however, so at least one of the two jets ends up at a large negative rapidity. The peak for the other jet at $y \approx 0$ is related to the structure of the $\gamma+q \rightarrow q+g$ matrix element. The VMD events are more symmetric, but one observes that the photon, i.e. pion, structure functions are harder than the proton ones. The asymmetry is even larger between the anomalous photon and the proton structure functions, as should be. Therefore model II is also the one most tilted towards activity in the photon hemisphere. Model III is surprisingly symmetric. This is related to the small- $x$ behaviour of the gluon structure function of the photon, which is here Drees-Grassie. If one instead uses Gordon-Storrow LO1 the distribution is markedly shifted towards the photon hemisphere, although still not as much as in model II. The $\sum E_{\perp}$ distribution is not noticeably affected by this switch of structure functions.

Naïvely one would expect the absence of a photon beam jet in direct events to have consequences for the energy flow in the photon direction. As is seen in Fig. 16, this is not really the case. The reason is that the lower cut-off for direct processes, $p_{0}=0.5 \mathrm{GeV}$, is not significantly larger than the typical primordial $k_{\perp}$ scale of VMD events. This is better shown in Fig. 17, where the $p_{\perp}$ of VMD and anomalous beam jets is compared with the $p_{\perp}$ of direct events. The good news is that the VMD class, on the one hand, and the direct and anomalous classes, on the other, smoothly join each other - remember that the step at $p_{0}=0.5 \mathrm{GeV}$ is just a technical artefact; one should rather think in terms of a smooth threshold at around this scale. This makes it credible that our model covers the full range of events, without any double-counting or holes. The bad news is that direct events lose their simple interpretation as high- $p_{\perp}$ ones without a photon beam remnant - many direct events are low- $p_{\perp}$ ones. Therefore the anomalous event class is the one with least energy flow in the photon direction: here the beam remnant has larger $p_{\perp}$ and smaller energy. We will come back to this issue later.

If all the charged particles of an event are ordered in rapidity, $y_{1}>y_{2}>\cdots>y_{n}$, one may define a maximum rapidity gap by $\Delta y_{\max }=\max _{i}\left(y_{i}-y_{i+1}\right)$. The distribution of this gap is found in Fig. 18. Not surprisingly, the elastic events show up as a sharp peak at large values, while diffractive events display a spectrum of values. This spectrum is damped at the lower end, where the other classes sit, so a smooth transition is achieved in the sum. The VMD non-diffractive, direct and anomalous events are all three rather similar, so models mainly differ by the amount of elastic and diffractive events, i.e. by the effective $4 \pi \alpha / f_{V}^{2}$ couplings. A study of rapidity gaps is thus one way to determine the latter. Obviously it would be necessary to cross-check that the events with large gaps also have the other properties expected in the model. 


\subsection{Jet events}

We now turn the attention to jet events. Such events are required to have at least one 'jet' with $E_{\perp j e t}=\sum E_{\perp}>10 \mathrm{GeV}$ within a cone size $R=\sqrt{(\Delta \eta)^{2}+(\Delta \phi)^{2}}<1$, as defined and found by the LUCELL algorithm [23].

The possibility of jet production in diffractive events has been neglected, so the three inclusive jet rates obtained are $0.108 \mu \mathrm{b}$ for VMD, $0.074 \mu \mathrm{b}$ for direct and $0.375 \mu \mathrm{b}$ for anomalous. We already see here the important rôle played by the anomalous component.

The charged multiplicity distribution of jet events is shown in Fig. 19. Direct events have the lowest multiplicity, owing to the absence of a photon beam remnant, while VMD ones have the highest, owing to the presence of multiple interactions. In many respects it is natural to view anomalous events as interpolating between the properties of VMD events and those of direct ones. The appearance of the anomalous distribution in the middle between the other two is therefore a pattern that we will see repeated. In this sense jet event distributions are much more predictable than the inclusive ones. Among the models, those with an enhanced VMD component have larger multiplicity. Model III also gains in multiplicity by the profuse multiple interactions.

The transverse energy distribution in rapidity, $\mathrm{d} E_{\perp} / \mathrm{d} y$, Fig. 20, shows the expected behaviour clearly. The direct events are very asymmetric, tilted towards the $\gamma$ side of the event, with the anomalous ones a bit less so and the VMD ones rather central. One should compare with Fig. 15, where the same pattern is present, but overlayed with additional effects. Again model V, the pure VMD one, stands out.

The jet rate as a function of pseudorapidity, Fig. 21, shows the same pattern, i.e. the energy distribution $\mathrm{d} E_{\perp} / \mathrm{d} y$ is easily recognized as coming from the jet activity. For the bottom frame, the absolute cross section $\mathrm{d} \sigma_{\text {jet }} / \mathrm{d} \eta$ is given, so differences in both shape and rate are visible. In particular, the importance of the anomalous component is apparent.

The jet rate as a function of $E_{\perp \text { jet }}$, Fig. 22, illustrates the differences in matrix elements and structure functions. The matrix elements of direct processes basically behave like $\mathrm{d} p_{\perp}^{2} / p_{\perp}^{2}$. Combined with the $\delta(1-x)$ direct structure function, this gives the slowest dropoff of jet rate. At sufficiently large $E_{\perp j e t}$ the direct processes therefore always win out. The VMD and anomalous processes both have a matrix element behaviour following $\mathrm{d} p_{\perp}^{2} / p_{\perp}^{4}$, but the anomalous photon structure function is harder and is increasing logarithmically with $p_{\perp}$ scale. Therefore VMD processes have the steepest $E_{\perp \text { jet }}$ variation. The bottom frame of Fig. 22 is in absolute cross-section units, as in Fig. 21, and shows the same differences between models.

The behaviour of the energy flow $\mathrm{d} E / \mathrm{d} \theta$ around the photon direction in inclusive events, Fig. 16, was not quite the expected one. It is then comforting to note that the traditional picture is recovered in jet events, Fig. 23: the direct events have no photon beam jets, while the VMD ones do. The anomalous processes are in between. However, this average behaviour does not tell the full story. Some anomalous events have their 'beam jets' at large angle and therefore look like direct events (in this region), while others have beam jets at small angle and look almost like VMD ones. Even the VMD class itself shows large fluctuations from one event to the next, e.g. depending on how much energy is carried away by the hard jets. As may be seen from Fig. 23, the energy flow in the photon direction shows large differences between the models, and so is a very useful quantity.

Finally, Fig. 24 shows the energy flow outside the jet core, in the 'pedestal', $1 \leq$ 
$\left|\eta-\eta_{j e t}\right| \leq 2,\left|\phi-\phi_{j e t}\right| \leq \pi / 2[26]$. Direct events are cleanest, as should be expected. Anomalous events receive extra contributions from the photon beam jet, and VMD ones also from multiple interactions. Here results are sensitive to the details of the multiple interactions scenario; if a model with varying impact parameter is used, the VMD pedestal $E_{\perp}$ will be even larger. Models do show a separation. In particular, in model III, with anomalous +VMD unitarized, also would-have-been anomalous jets can have underlying multiple interactions, and so the typical pedestal $E_{\perp}$ is larger than in model I.

\subsection{Multijet events}

Classification of events into a given number of jets is not unique, because of higher-order QCD corrections (parton showers), fragmentation, decays, primordial $k_{\perp}$, detector imperfections, ambiguities in jet-finding algorithms, and so on. An event nominally with two high- $p_{\perp}$ partons may well be reconstructed as a one-jet or as a three-jet event, depending on circumstances. If we forget such complications for a moment, the various event classes have the following structure:

- Direct events formally contain two high- $p_{\perp}$ jets and one beam jet, $2+1$ for short. Because of the low cut-off scale $p_{0}$ the two 'high- $p_{\perp}$ ' jets very often appear as a single beam jet in the forward direction, giving $0+2$.

- VMD low- $p_{\perp}$ events are $0+2$, while high- $p_{\perp}$ ones are $2+2$. Multiple interactions add the possibility of further jet pairs, i.e. $4+2,6+2$, and so on. Multiple interactions could also involve $3 \rightarrow 3$ processes (one parton which consecutively scatters against two partons from the other hadron) to give an odd number of jets, but this rate is supposed to be lower and is neglected here.

- An anomalous high- $p_{\perp}$ event is either $3+1$ or $2+2$, depending on whether the photon 'remnant jet' has a large or small $p_{\perp}$. At lower $p_{\perp}$, events appear as $1+2$ or $0+2$. Note that this is the only process where there may be one single high- $p_{\perp}$ jet already at a very naïve parton level, since the $p_{\perp}$ recoil here can be split between two other jets which each fall below the jet threshold.

It would seem that some simple jet counting would give a handle on the three components. In particular, it would allow us to find multiple interactions in VMD events, simply by looking for events with four or more jets. However, the smearing effects mentioned above are very important; QCD showers, in particular, may easily give rise to additional jets. Therefore the study of multiple interactions is difficult already in a purely hadronic environment (i.e. at $\bar{p}$ p colliders). To this one must now add the direct and anomalous 'backgrounds'. These latter have a less steep $p_{\perp}$ spectrum, which translates into an enhanced possibility for a pair of two jets at large $p_{\perp}$ to shower into four jets of medium large $p_{\perp}$.

To study multijet rates, we decrease the jet threshold to $E_{\perp j e t}>5 \mathrm{GeV}$ and the cone size to $R<0.7$. This gives the results in the upper half of Table 1 . The parton shower approach contains uncertainties, so absolute numbers should be taken with a pinch of salt, but the general trend should be representative of what is to be expected. As discussed above, the multijet rate in VMD events is increased if one assumes variable impact parameters in the multiple interactions scenario, because of the large importance of central collisions. Even so, there are more anomalous multijet events than VMD ones.

The multiple interaction rate increases in relative importance if low- $p_{\perp}$ jets can be observed. Therefore we also study a more extreme case, lower half of Table 1, wherein jets are allowed down to $E_{\perp \text { jet }}=3 \mathrm{GeV}$, although one jet is still required to have $E_{\perp j e t}>$ 
$5 \mathrm{GeV}$. This is probably as low as one can go, without having too many jets just from fluctuations in the fragmentation. Here the enhanced multiple interactions scenario and the anomalous event class give comparable numbers of events with four or more jets. Cuts on beam jet structure and requirements of pairwise balancing jets could be used to enhance the multiple-interactions signal relative to the background.

\subsection{Comments on model comparisons}

We have shown a few examples of interesting event properties. Generally, differences between the direct, VMD and anomalous components are seen to be significant. When the components are mixed in different proportions, however, differences may be more quantitative than qualitative. One can therefore not point to one single plot and claim that a measurement of that one is enough to pin down the right model. Understanding will be gained by a study of many different observables. Some models may be excluded by one observable, others by another. For instance, compared with our favoured scenario,

- model II has a different charged multiplicity distribution (Fig. 14) and a different energy flow in the photon direction (Fig. 16),

- model III has a larger charged multiplicity (Fig. 14), a larger energy flow in the photon direction (Fig. 23) and a larger pedestal $E_{\perp}$ (Fig. 24),

- model IV has a larger multiplicity in jet events (Fig. 19), a smaller jet rate (Figs. 21 and 22) and a different energy flow in the photon direction (Fig. 23), and

- model $\mathrm{V}$ is the one most different, in particular with respect to jet events.

One should keep in mind that our model does contain several uncertainties which, without invalidating any approach as such, still tends to make absolute predictions difficult. For instance, jet rates may need to be scaled up or down by an effective $K$-factor, i.e. by effects of higher orders (some of which may be contained in the parton shower description, but not all). Therefore tuning may be necessary, whereby some key quantities are seen to agree, before a model can be used to predict other observables. This will be a longer task. Probably many studies will be based on correlations between various observables, or on events within specific cuts, rather than on the inclusive distributions shown in this paper.

\section{Conclusions and Outlook}

If this paper is long, it is for a good reason: $\gamma p$ physics is far from simple. This nontriviality means that photoproduction physics at HERA has the potential of becoming very fruitful. At the end of the day, we may hope to have a much better understanding of the structure of a high-energy photon and its interactions with hadrons. The extraction of relevant quantities from the multihadronic $\gamma p$ samples is going to be a demanding task, however. It may be just about impossible without the guidance of models which, however imperfect, are still comprehensive and realistic enough to predict the bulk of observable physics. We have in this paper presented the first complete model that contains the direct and hadron-like interactions of the photon with protons and aims at a smooth matching of soft and hard interactions. It would be next to a miracle if this particular scenario survived without a need for further refinements, but at least our model might be a good starting point. We have also given alternatives, to illustrate how experimental information would help distinguish the details of the underlying physics. 
It is useful to summarize the highlights of our model. Firstly, we have developed a number of tools:

- New parametrizations of $\gamma p$ and $p p / \bar{p} p$ elastic, single diffractive and double diffractive cross sections, in both differential and integrated form.

- A scheme for extending any given proton structure function parametrization into the regions of small $x$ and/or $\mu^{2}$.

- A new parametrization of the photon structure function, in which the VMD and anomalous parts are clearly separated.

Secondly, we have emphasized a number of concepts, some of them new:

- The superposition of the physical photon state, made up of direct, VMD and anomalous components, eq. (3).

- The interactions of each of the states with hadrons.

- The structure of complete final state for each case, including predictions for the photon remnants.

- Matching of the three event classes and prediction of their relative weights.

- A low transverse-momentum cut-off scale $p_{0} \approx 0.5 \mathrm{GeV}$ to distinguish perturbative $\gamma q \bar{q}$ vertices (direct, anomalous) from non-perturbative ones (VMD).

- Unitarization of the VMD jet rate, i.e. the possibility of having multiple partonparton interactions in an event.

- Non-unitarization of anomalous events. Here the jet rate is instead kept in check by the assumption of a smaller spatial extent of anomalous $\gamma \leftrightarrow q \bar{q}$ fluctuations than of VMD ones, which translates into different $p_{\perp \text { min }}$ cut-offs.

One should not be blind to the uncertainties of this modelling. Here is a list of only a few:

- Our model is a probabilistic one. Would a more sophisticated amplitude-level description lead to observable interference effects?

- How far can a VMD ansatz be trusted, i.e. how closely does a 'VMD $\rho$ ' agree with a 'true $\rho^{0}$ ' in its properties, such as structure functions?

- The $4 \pi / f_{V}^{2}$ VMD couplings are essential for our description, and yet their experimental determination is uncertain by a fair amount. It is highly desirable to measure the reactions $\gamma p \rightarrow V$ p at high energies, in particular to establish (or disprove) their energy independence.

- The naïve pomeron language leads to inconsistencies for elastic and diffractive event rates. How reliable are our proposed modifications?

- Can one really use perturbation theory to describe jet production down to transverse momenta as low as $p_{0} \approx 0.5 \mathrm{GeV}$ ?

- If so, how reliable is our proton structure function extension in this region?

- How large are higher-order corrections to jet rates? Is the parton-shower approximation sufficiently good?

- Does unitarization really work the same way in VMD processes as in p p interactions?

- How much is our procedure, to determine $p_{\perp \text { min }}^{\text {anom }}(s)$ from 'what is left' in the cross section, based on wishful thinking?

- Should $p_{\perp \text { min }}$ cut-off scales be energy-dependent or not?

- Have we missed some important class of events?

Furthermore, our attempt has been to cover all major kinds of events, as required for a study of inclusive event samples. The flip side of the coin is that we have not spent much 
time on problems of exclusive event types. Some issues have only partly been included, as charm production, or not at all, as jet production in diffractive events. The program therefore has its limits.

Clearly, many issues remain to be answered experimentally. A first round includes the following:

- What is $\sigma_{\text {tot }}^{\gamma \mathrm{p}}(s)$ ?

- Is there evidence for each of the three proposed event classes?

- If so, what is the relative composition of event classes?

- Can the different $p_{\perp}$ cut-off scales be determined experimentally?

- Is there evidence for multiple interactions in VMD events? Might it even be present in anomalous events (cf. hot spots)?

- What is the structure of the photon remnant in the different event classes?

As these questions are answered, no doubt they will be replaced by others, of increasing complexity.

For the future, we see two obvious areas of further study. One is the transition from photoproduction to deep inelastic scattering. Our model only covers the $Q^{2} \ll m_{\rho}^{2}$ limit. The other is the application of our photon picture to $\gamma \gamma$ events. Here data already exist from PETRA, PEP and TRISTAN, although typical $\gamma \gamma$ masses are rather small. LEP II and future linear $\mathrm{e}^{+} \mathrm{e}^{-}$colliders (the latter maybe even with dedicated $\gamma \gamma$ modes based on back-scattered laser beams) should make it possible to perform much more precise tests. The study of photon physics should therefore continue to be a rich field, theoretically and experimentally.

\section{References}

[1] G.A. Schuler and T. Sjöstrand, Phys. Lett. B300 (1993) 169

[2] J.J. Sakurai, Ann. Phys. (N.Y.) 11 (1960) 1;

M. Gell-Mann and F. Zachariasen, Phys. Rev. 124 (1961) 953

[3] T.H. Baur et al., Rev. Mod. Phys. 50 (1978) 261;

'Electromagnetic Interactions of Hadrons', eds. A. Donnachie and G.Shaw (Plenum Press, New York, 1978)

[4] For a recent review see e.g. G. Wormser, Orsay preprint LAL 90-37, presented at the Workshop on Vector-Meson Dominance Phenomena, Göttingen, 1990

[5] 'Proceedings of the HERA Workshop', ed. R.D. Peccei (DESY, Hamburg, 1988)

[6] 'Physics at HERA', eds. W. Buchmüller and G. Ingelman (DESY, Hamburg, 1992)

[7] C. Berger and W. Wagner, Phys. Rep. 146 (1987) 1;

H. Kolanoski and P. Zerwas, in 'High Energy Electron-Positron Physics', eds. A. Ali and P. Söding (World Scientific, Singapore, 1988);

J.H. Da Luz Vieira and J.K. Storrow, Z. Phys. C51 (1991) 241

[8] F. Borzumati and G.A. Schuler, 'Real and virtual photon contributions to inelastic ep scattering', DESY 92-078, to appear in Z. Physik C

[9] AMY collaboration, R. Tanaka et al., Phys. Lett. B277 (1992) 215 
[10] G.A. Schuler, in ref. [6], Vol. 1, p. 461, and references therein

[11] ZEUS Collaboration, M. Derrick et al., Phys. Lett. B293 (1992) 465

[12] H1 Collaboration, T. Ahmed et al., DESY 92-160

[13] ZEUS Collaboration, M. Derrick et al., DESY 92-138

[14] H1 Collaboration, T. Ahmed et al., DESY 92-142

[15] G. Ingelman, Computer Physics Commun. 46 (1987) 217;

G. Ingelman and A. Weigend, Computer Physics Commun. 46 (1987) 241;

G. Marchesini, B.R. Webber, M.H. Seymour, G. Abbiendi, L. Stanco and I.G. Knowles, Computer Physics Commun. 67 (1992) 465

[16] See for instance Proc. 'Small- $x$ behaviour of deep inelastic structure functions in QCD', DESY, Hamburg, 1990, eds. A. Ali and J. Bartels, Nucl. Phys. B (Proc. Suppl) 18C (1990)

and Proc. 'Deep Inelastic Scattering', Teupitz, Germany, 1992, eds. J. Blümlein and T. Riemann, Nucl. Phys. B (Proc. Suppl.) 29A (1992)

[17] H.T. Nieh, Phys. Rev. D1 (1970) 3161

[18] P. Aurenche et al., in ref. [5], Vol. 2, p. 561

[19] T. Sjöstrand, FERMILAB-PUB-85/119-T (1985);

T. Sjöstrand and M. van Zijl, Phys. Rev. D36 (1987) 2019

[20] UA5 Collaboration, R.E. Ansorge et al., Z. Phys. C43 (1989) 357

[21] L. Durand and H. Pi, Phys. Rev. Lett. 58 (1987) 303

[22] H.-U. Bengtsson and T. Sjöstrand, Computer Physics Commun. 46 (1987) 43

[23] T. Sjöstrand, CERN-TH.6488/92 (1992)

[24] Z. Koba, H.B. Nielsen and P. Olesen, Nucl. Phys. B40 (1972) 317

[25] UA5 Collaboration, R.E. Ansorge et al., Z. Phys. C37 (1988) 191

[26] UA1 Collaboration, C. Albajar et al., Nucl. Phys. B309 (1988) 405

[27] UA1 Collaboration, C. Albajar et al., Nucl. Phys. B335 (1990) 261

[28] AFS Collaboration, T. Åkesson et al., Z. Phys. C34 (1987) 163 ; UA2 Collaboration, J. Alitti et al., Phys. Lett. B268 (1991) 145 ;

L. Keeble (CDF Collaboration), FERMILAB-CONF-92-161-E

[29] Particle Data Group, K. Hikasa et al., Phys. Rev. D45 (1992) S1

[30] P.D.B. Collins, 'An introduction to Regge theory and high energy physics' (Cambridge University Press, 1977)

[31] A. Donnachie and P.V. Landshoff, CERN-TH.6635/92 
[32] M. Froissart, Phys. Rev. 123 (1961) 1053;

A. Martin, Phys. Rev. 124 (1963) 1432

[33] E.M. Levin and L.L. Frankfurt, JETP Letters 2 (1965) 65;

H.J. Lipkin and F. Scheck, Phys. Rev. Lett. 16 (1966) 71;

J.J.J. Kokkedee, 'The Quark Model' (W.A. Benjamin, New York, 1969)

[34] O. Nachtmann, Ann. Phys. 209 (1991) 436

[35] A. Donnachie and P.V. Landshoff, Nucl. Phys. B267 (1986) 690

[36] J. Pumplin, Phys. Rev. D8 (1973) 2899

[37] G.A. Schuler and T. Sjöstrand, in preparation

[38] K. Goulianos, Phys. Rep. 101 (1983) 169

[39] G. Wolf, Nucl. Phys. B26 (1971) 317

[40] H. Plothow-Besch, CERN-PPE/92-123, and references therein

[41] G. Parisi and R. Petronzio, Phys. Lett. 62B (1976) 331;

V.A. Novikov et al., JETP Lett. 24 (1976) 341; Ann. Phys. 105 (1977) 276;

M. Glück and E. Reya, Nucl. Phys. B130 (1977) 76

[42] M. Glück, E. Reya and A. Vogt, Z. Phys. C48 (1990) 471 and C53 (1992) 127

[43] NMC Collaboration, P. Amaudruz et al., Phys. Lett. B295 (1992) 159

[44] A. Donnachie and P.V. Landshoff, Nucl. Phys. B244 (1984) 322;

P.V. Landshoff, Nucl. Phys. B (Proc. Suppl.) 18C (1990) 211

[45] H. Abramowicz, E. Levin, A. Levy and U. Maor, Phys. Lett. B269 (1991) 465

[46] E.M. Levin and M.G. Ryskin, Nucl. Phys. B (Proc. Suppl.) 18C (1990) 92

[47] L.V. Gribov, E.M. Levin and M.G. Ryskin, Phys. Rep. 100 (1983) 1;

A.H. Mueller and J. Qiu, Nucl. Phys. B268 (1986) 427;

J. Bartels, J. Blümlein and G.A. Schuler, Z. Phys. C50 (1991) 91;

J. Kwiecinski, A.D. Martin, W.J. Stirling and R.G. Roberts, Phys. Rev. D42 (1990) 3645

[48] G.A. Schuler, in preparation

[49] P.J. Sutton, A.D. Martin, R.G. Roberts and W.J. Stirling, Phys. Rev. D45 (1992) 2349

[50] A.H. Mueller and H. Navalet, Nucl. Phys. B282 (1987) 727

[51] T. Sjöstrand, Computer Physics Commun. 39 (1986) 347;

T. Sjöstrand and M. Bengtsson, Computer Physics Commun. 43 (1987) 367

[52] G. Ingelman and P.E. Schlein, Phys. Lett. 152B (1985) 256;

UA8 Collaboration, A. Brandt et al., CERN PPE/92-179 
Table 1: Exclusive jet cross sections. The first three columns are for VMD events, without multiple interactions or with them in standard scenario or in scenario with enhanced rate due to varying impact parameters. The next two columns are for direct and anomalous events, and the last one for the VMD+unitarized component of model III. The latter could be combined with either multiple interactions scenario; here we have used the standard one.

\begin{tabular}{|c|c|c|c|c|c|c|}
\hline $\begin{array}{c}\sigma \\
{[\mu \mathrm{b}]}\end{array}$ & $\begin{array}{l}\text { VMD } \\
\text { none }\end{array}$ & $\begin{array}{l}\text { multiple } i \\
\text { standard }\end{array}$ & $\begin{array}{l}\text { ractions }= \\
\text { enhanced }\end{array}$ & Direct & Anomalous & $\begin{array}{c}\text { Unitarized } \\
\text { VMD+anom. }\end{array}$ \\
\hline \multicolumn{7}{|c|}{ Each jet has $E_{\perp j e t}>5 \mathrm{GeV}$ in $R<0.7$ cone } \\
\hline 1 jet & 0.75 & 0.97 & 1.20 & 0.20 & 1.69 & 2.78 \\
\hline 2 jets & 0.20 & 0.26 & 0.33 & 0.15 & 0.67 & 0.90 \\
\hline 3 jets & 0.024 & 0.027 & 0.042 & 0.024 & 0.15 & 0.12 \\
\hline 4 jets & 0.001 & 0.003 & 0.004 & 0.002 & 0.022 & 0.012 \\
\hline 5 jets & 0.000 & 0.000 & 0.000 & 0.000 & 0.002 & 0.001 \\
\hline \multicolumn{7}{|c|}{ One jet has $E_{\perp \text { jet }}>5 \mathrm{GeV}$, the rest have $E_{\perp \text { jet }}>3 \mathrm{GeV}, R<0.7$ cone } \\
\hline 1 jet & 0.25 & 0.31 & 0.37 & 0.054 & 0.52 & 0.82 \\
\hline 2 jets & 0.45 & 0.56 & 0.66 & 0.21 & 1.08 & 1.68 \\
\hline 3 jets & 0.13 & 0.18 & 0.25 & 0.072 & 0.52 & 0.65 \\
\hline 4 jets & 0.021 & 0.030 & 0.084 & 0.013 & 0.14 & 0.18 \\
\hline 5 jets & 0.003 & 0.007 & 0.026 & 0.002 & 0.029 & 0.032 \\
\hline 6 jets & 0.000 & 0.001 & 0.004 & 0.000 & 0.004 & 0.008 \\
\hline
\end{tabular}




\section{Figure captions}

Fig. 1 A photon may fluctuate into q $\bar{q}$ pairs. Low-virtuality fluctuations are nonperturbative and give intermediate VMD states, while high-momentum fluctuations are perturbatively described.

Fig. 2 Contributions to hard $\gamma$ p interactions: a) direct, b) VMD, and c) anomalous. Only the basic graphs are illustrated; additional partonic activity is allowed in either case.

Fig. 3 The total $\gamma$ p cross section $\sigma_{\text {tot }}^{\gamma \mathrm{p}}(s)$, top full curve, and the VMD one $\sigma_{\mathrm{VMD}}^{\gamma \mathrm{p}}(s)$, full curve below that. Within the VMD cross section, the various components are separated by dashed lines, from bottom to top: elastic, single diffractive $(V X)$, single diffractive $(X \mathrm{p})$, double diffractive, and non-diffractive.

Fig. 4 Schematic picture of how the $\left(x, \mu^{2}\right)$ plane is subdivided into four different regions for our extension of the standard proton structure functions. See text for details.

Fig. 5 The g, d, $u$ and s structure functions of the proton. The full line is for MRS S0 at $\mu^{2}=\mu_{0}^{2}=5 \mathrm{GeV}^{2}$, dashed our modified behaviour at the same scale, and dash-dotted our extension to $\mu^{2}=0.5 \mathrm{GeV}^{2}$.

Fig. 6 The g, $d, u$ and $s$ structure functions of the photon at $\mu^{2}=5 \mathrm{GeV}^{2}$. The full line is for $f_{a}^{\gamma, \text { res }}$ of Drees-Grassie, which should be compared with the sum of $f_{a}^{\gamma, \mathrm{VMD}}$ (based on Owens 1 for the $\pi^{+}$), dashed, and $f_{a}^{\gamma, \text { anom }}$ of Schuler, dash-dotted.

Fig. 7 The integrals $\mathcal{I}^{\gamma, \text { res }}\left(\mu^{2}\right)$, top figure, and $\mathcal{I}_{[0.2 .0 .8]}^{\gamma, \text { res }}\left(\mu^{2}\right)$, bottom one. The dashed curve is for $f_{a}^{\gamma, \mathrm{VMD}}$ and the full one for $f_{a}^{\gamma, \mathrm{VMD}}+f_{a}^{\gamma, \text { anom }}$ in our approach. The various symbols show corresponding results for a number of photon structure function sets: GRV LO filled squares, GS LO1 open triangles, GS LO2 diamonds, DG filled circles, LAC 2 open circles, LAC 1 stars, LAC 3 open squares and DO filled triangles (order from bottom to top at right edge of top figure). Some parametrizations are not valid all the way down to $1 \mathrm{GeV}^{2}$, and therefore appear flat at small $\mu^{2}$.

Fig. 8 Hard interaction rate as a function of c.m. energy. The top dashed curve gives the total interaction rate, with the intermediate dashed ones separating the three components, from the bottom VMD, direct and anomalous. For reference, the full curve is the total cross section, cf. Fig. 3.

Fig. 9 Event rates as a function of c.m. energy. Curves as in Fig. 3. In addition the top two dashed curves represent VMD+direct and VMD+direct+anomalous. Same $p_{\perp \text { min }}$ cut-off in VMD and anomalous events.

Fig. 10 Curves as in Fig. 9, but with larger $p_{\perp \text { min }}$ cut-off in anomalous events, $p_{\perp m i n}^{\text {anom }}>$ $p_{\perp \min }^{\mathrm{VMD}}$.

Fig. 11 The five (six) scenarios for event composition discussed in the text. 
Fig. 12 Event rate as a function of the hard $p_{\perp}$ scale of processes (unobservable). Top figure: VMD elastic+diffractive dotted, VMD non-diffractive full, direct dashed, anomalous dash-dotted.

Bottom figure: model I full, model II dash-dotted, model III dashed, model IV large dots, model $\mathrm{V}$ dotted.

Fig. 13 Distribution of the total transverse energy $\sum E_{\perp}$ of events. Notation as in Fig. 12.

Fig. 14 Charged-multiplicity distribution. Notation as in Fig. 12.

Fig. 15 Transverse-energy distribution as a function of rapidity, $\mathrm{d} E_{\perp} / \mathrm{d} y$. Notation as in Fig. 12.

Fig. 16 Energy flow $\mathrm{d} E / \mathrm{d} \theta$ around the photon direction. Notation as in Fig. 12.

Fig. 17 Transverse momentum of 'remnant' jet on the parton level (unobservable). Notation as in Fig. 12.

Fig. 18 Largest rapidity gap present between two charged particles in an event. Notation as in Fig. 12.

Fig. 19 Charged-multiplicity distribution of jet events.

Top figure: VMD non-diffractive full, direct dashed, anomalous dash-dotted. Bottom figure: model I full, model II dash-dotted, model III dashed, model IV large dots, model $\mathrm{V}$ dotted.

Fig. 20 Transverse-energy distribution as a function of rapidity, $\mathrm{d} E_{\perp} / \mathrm{d} y$, for jet events. Notation as in Fig. 19.

Fig. 21 Jet rate as a function of jet pseudorapidity. Notation as in Fig. 19.

Fig. 22 Jet rate as a function of jet transverse energy. Notation as in Fig. 19.

Fig. 23 Energy flow $\mathrm{d} E / \mathrm{d} \theta$ around the photon direction in jet events. Notation as in Fig. 19.

Fig. 24 Total transverse energy in the 'pedestal', $1 \leq\left|\eta-\eta_{j e t}\right| \leq 2,\left|\phi-\phi_{j e t}\right| \leq \pi / 2$, of jet events. Notation as in Fig. 19. 


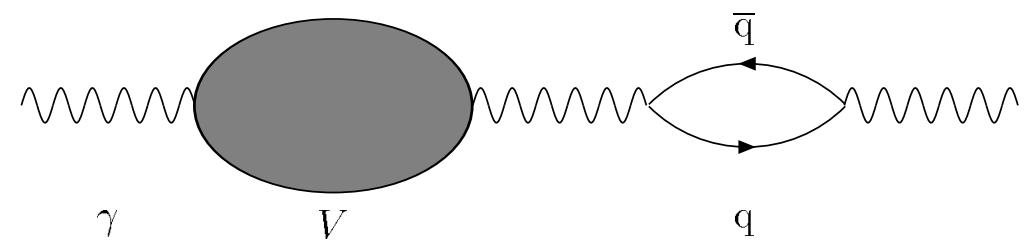

Fig. 1

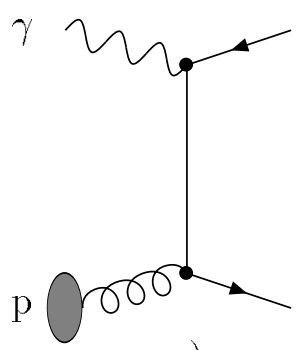

a)

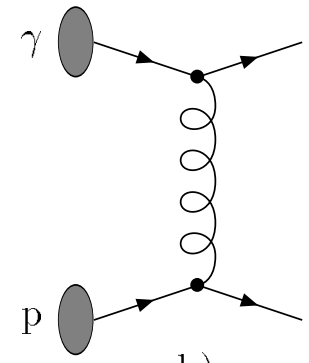

b)

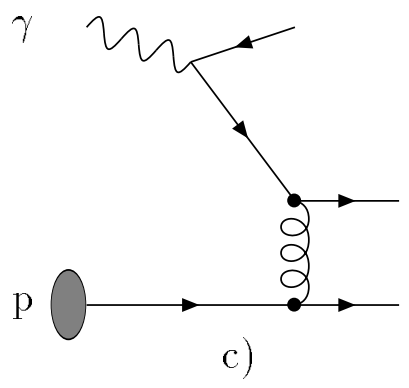

Fig. 2 


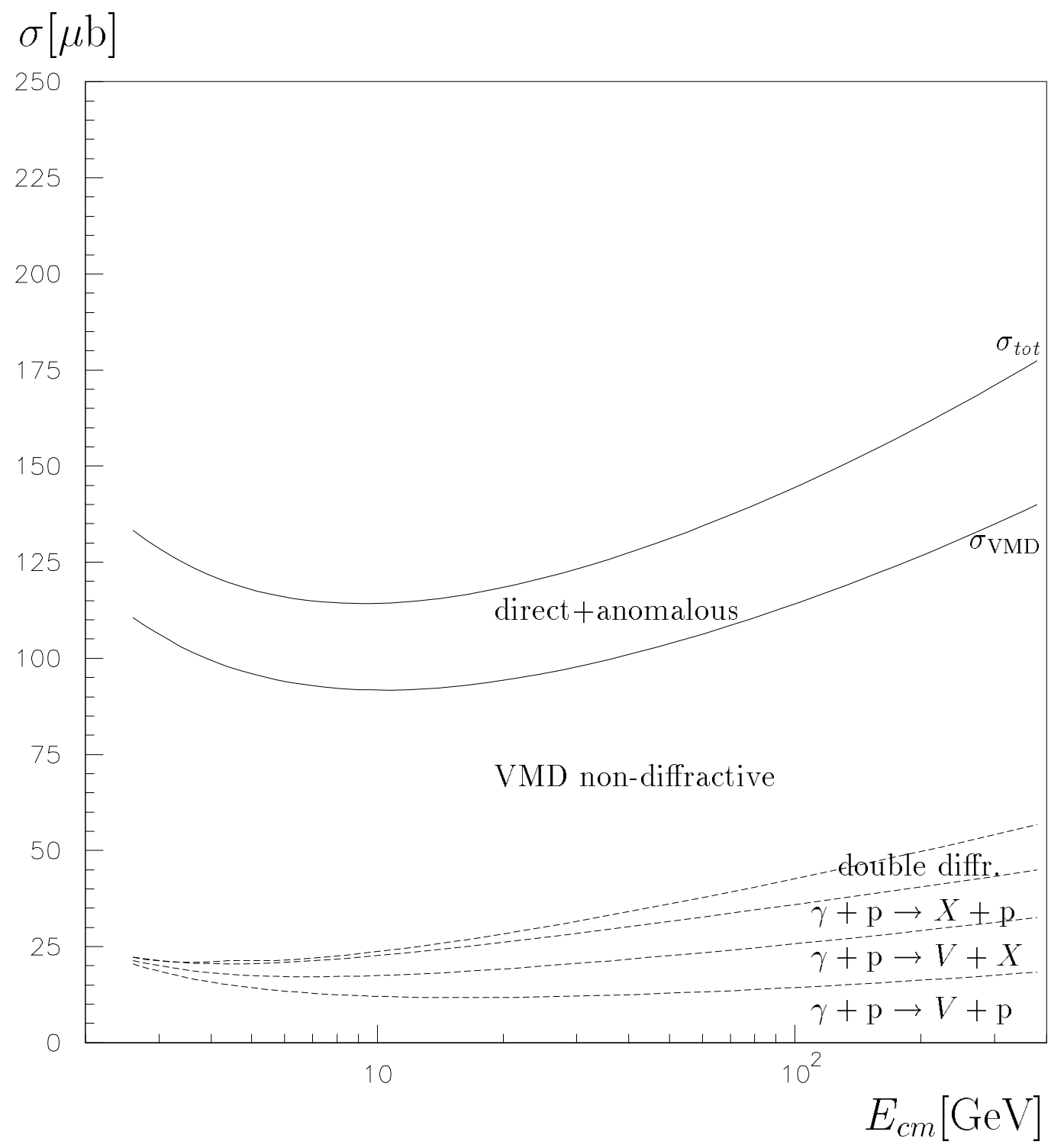

Fig. 3 


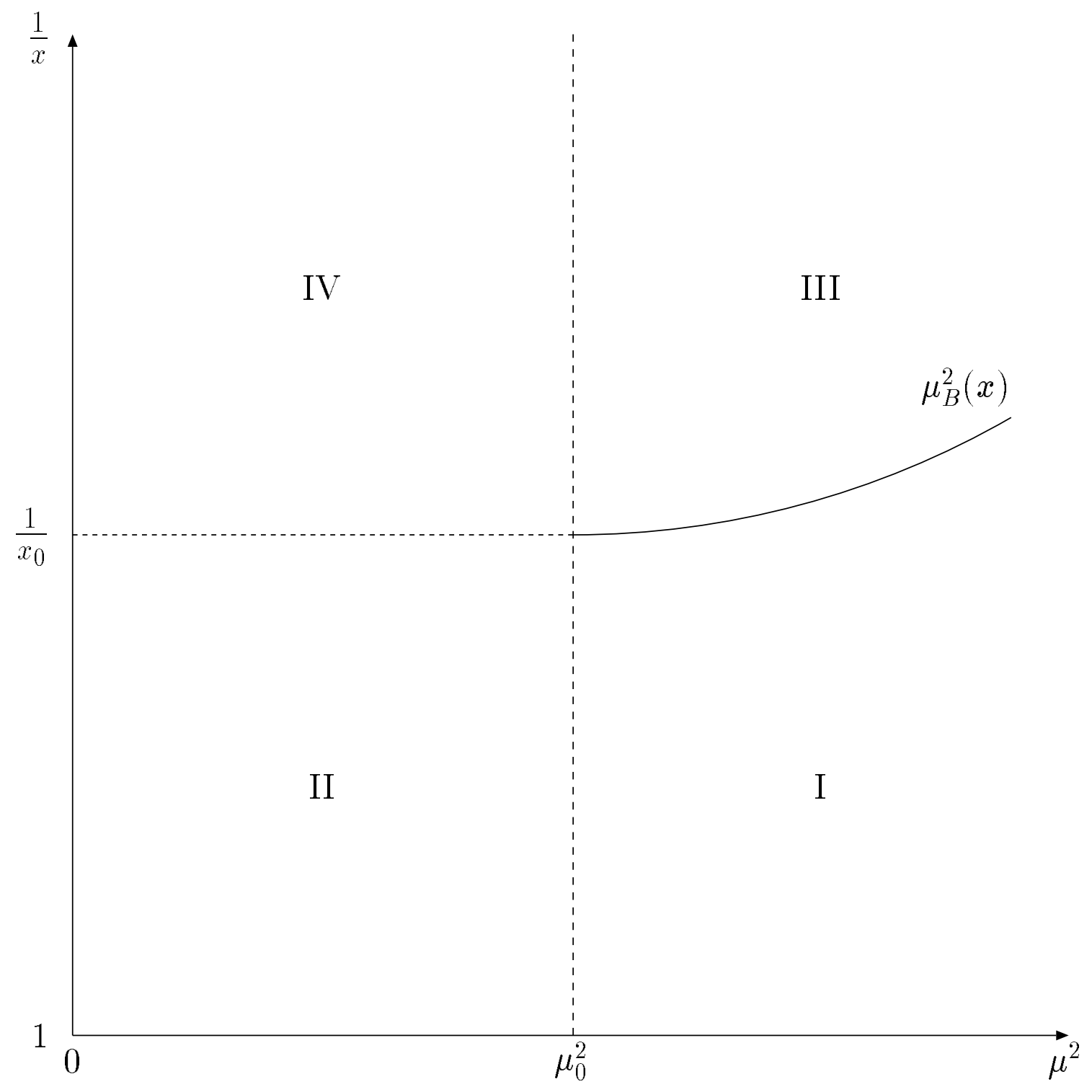

Fig. 4 

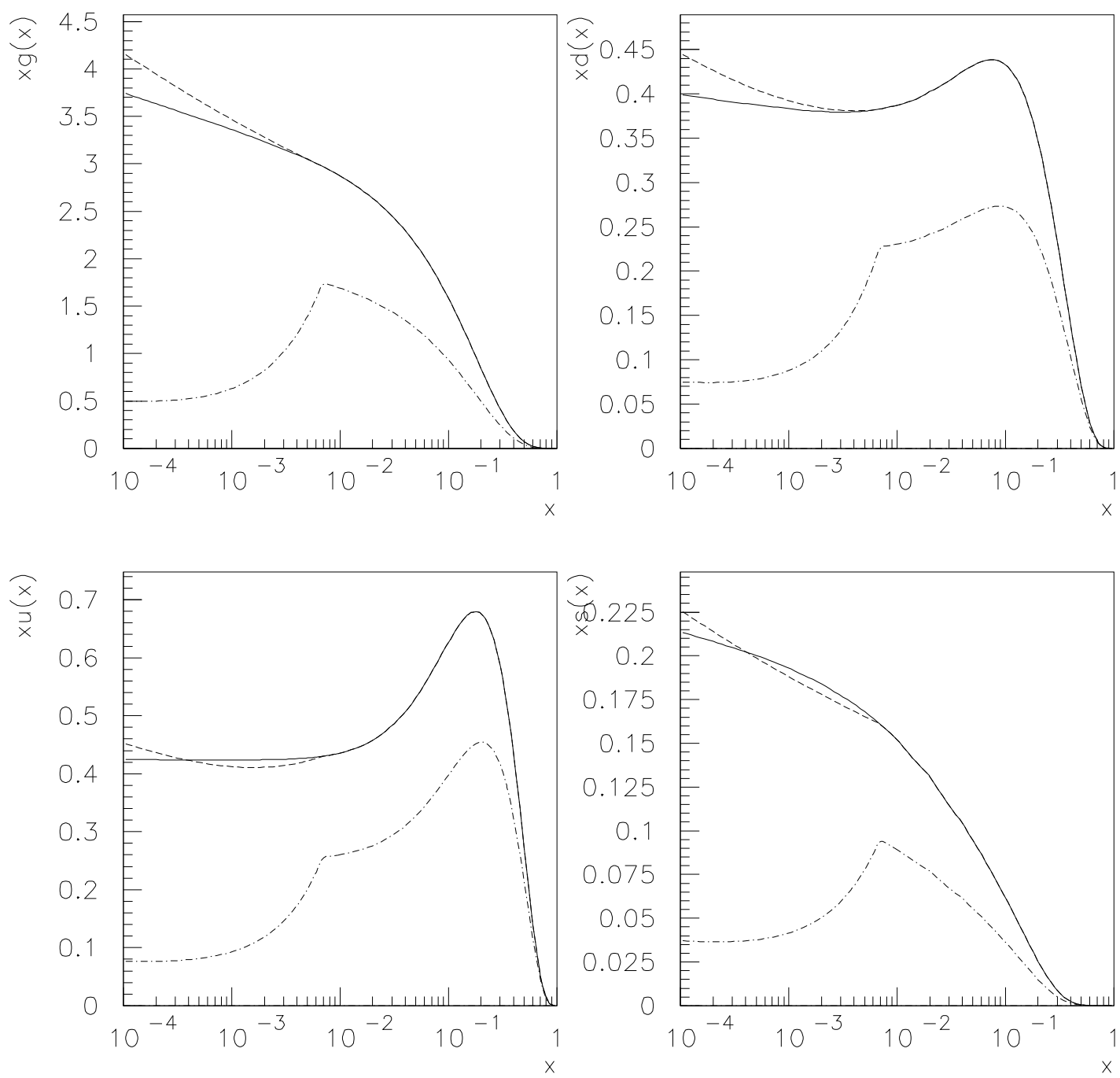

Fig. 5 

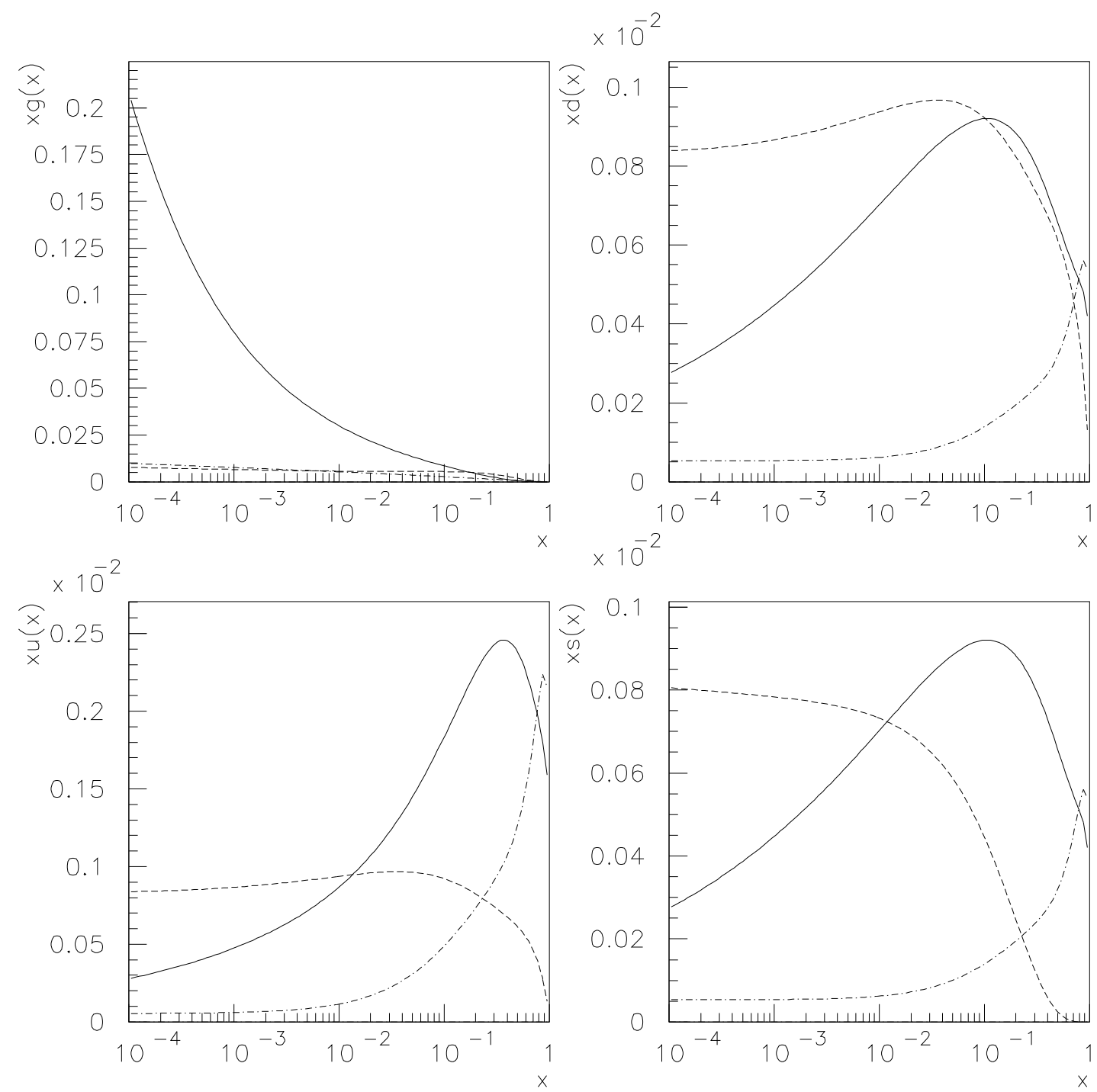

Fig. 6 

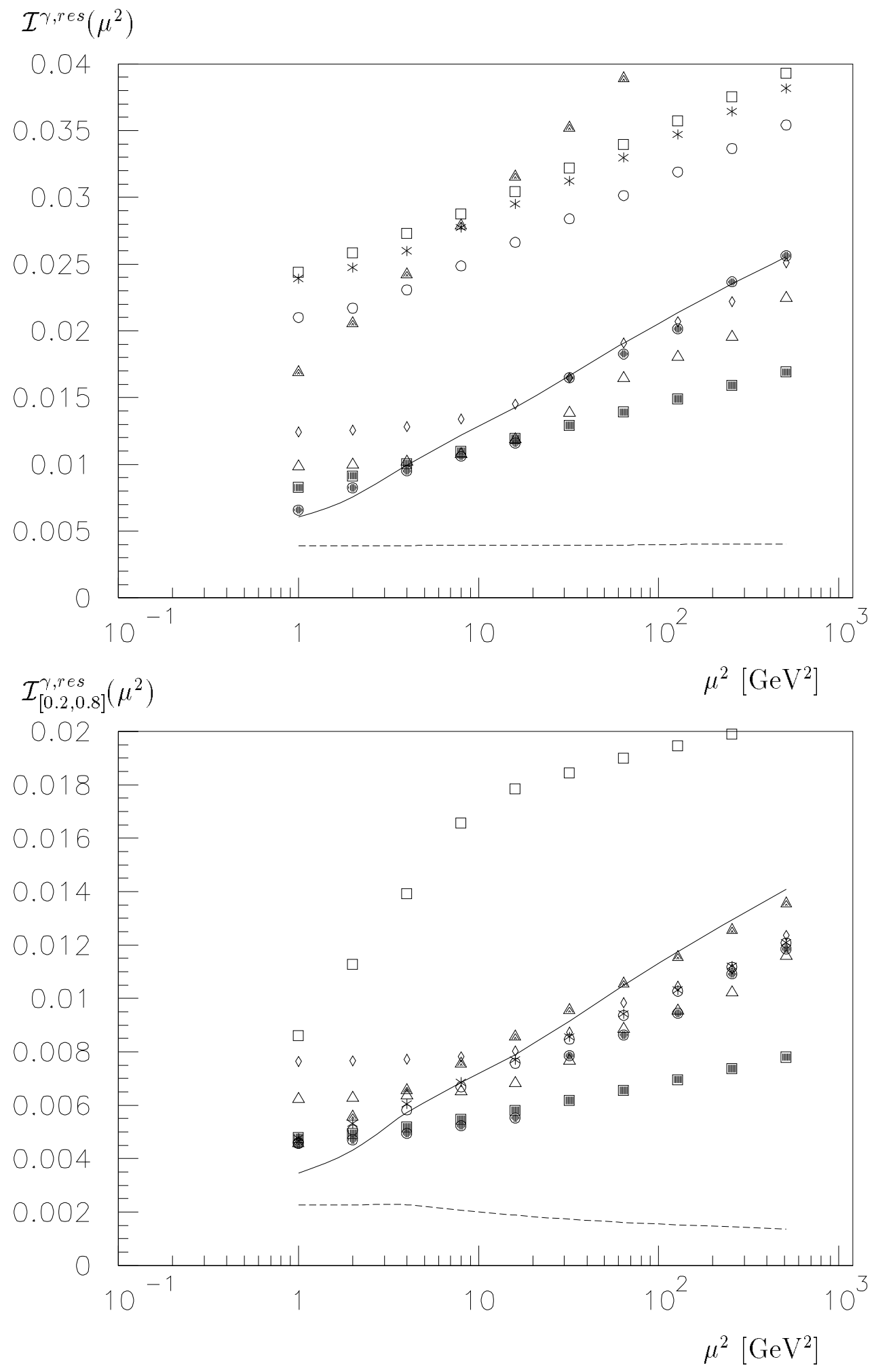

Fig. 7 


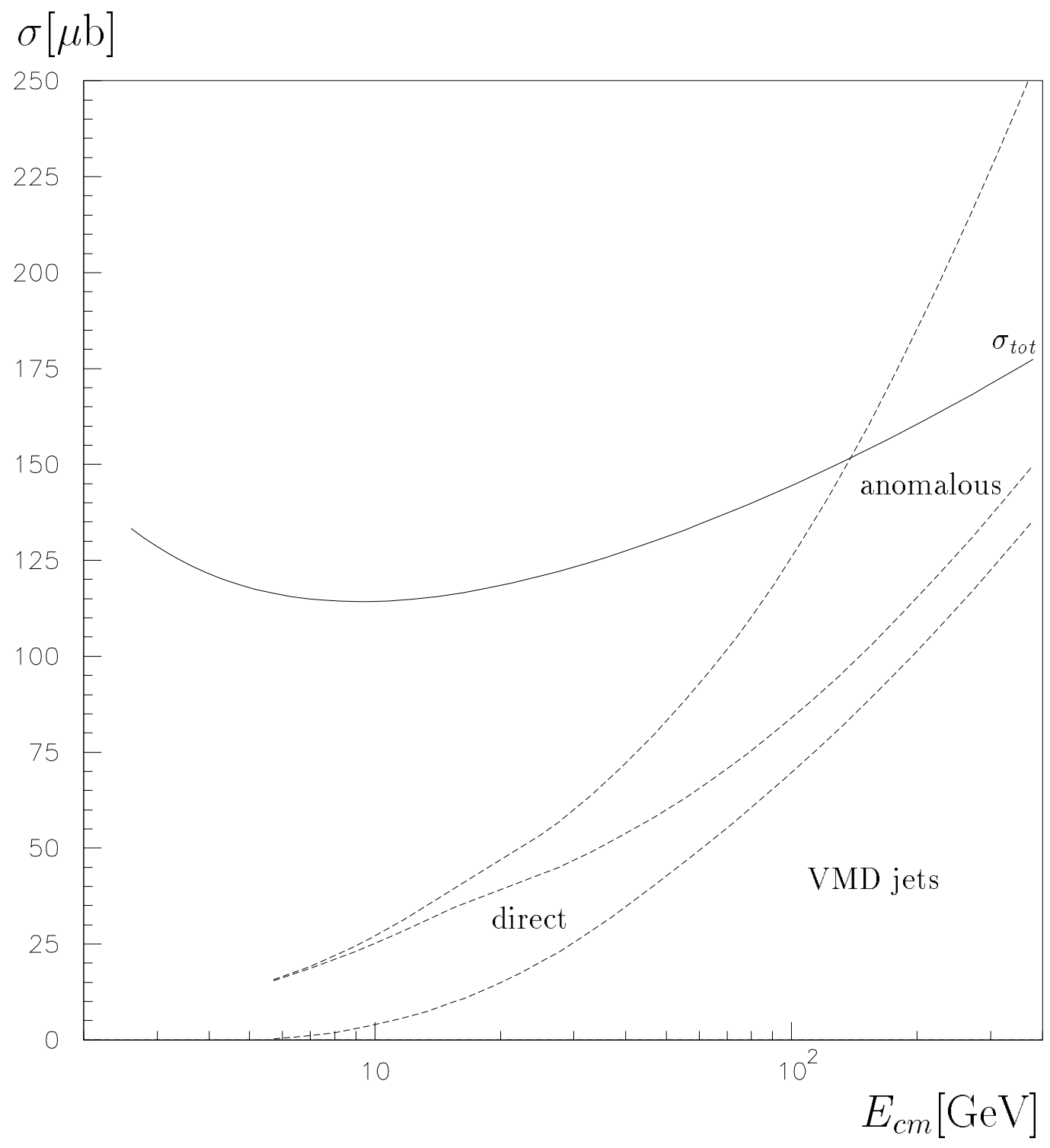

Fig. 8 


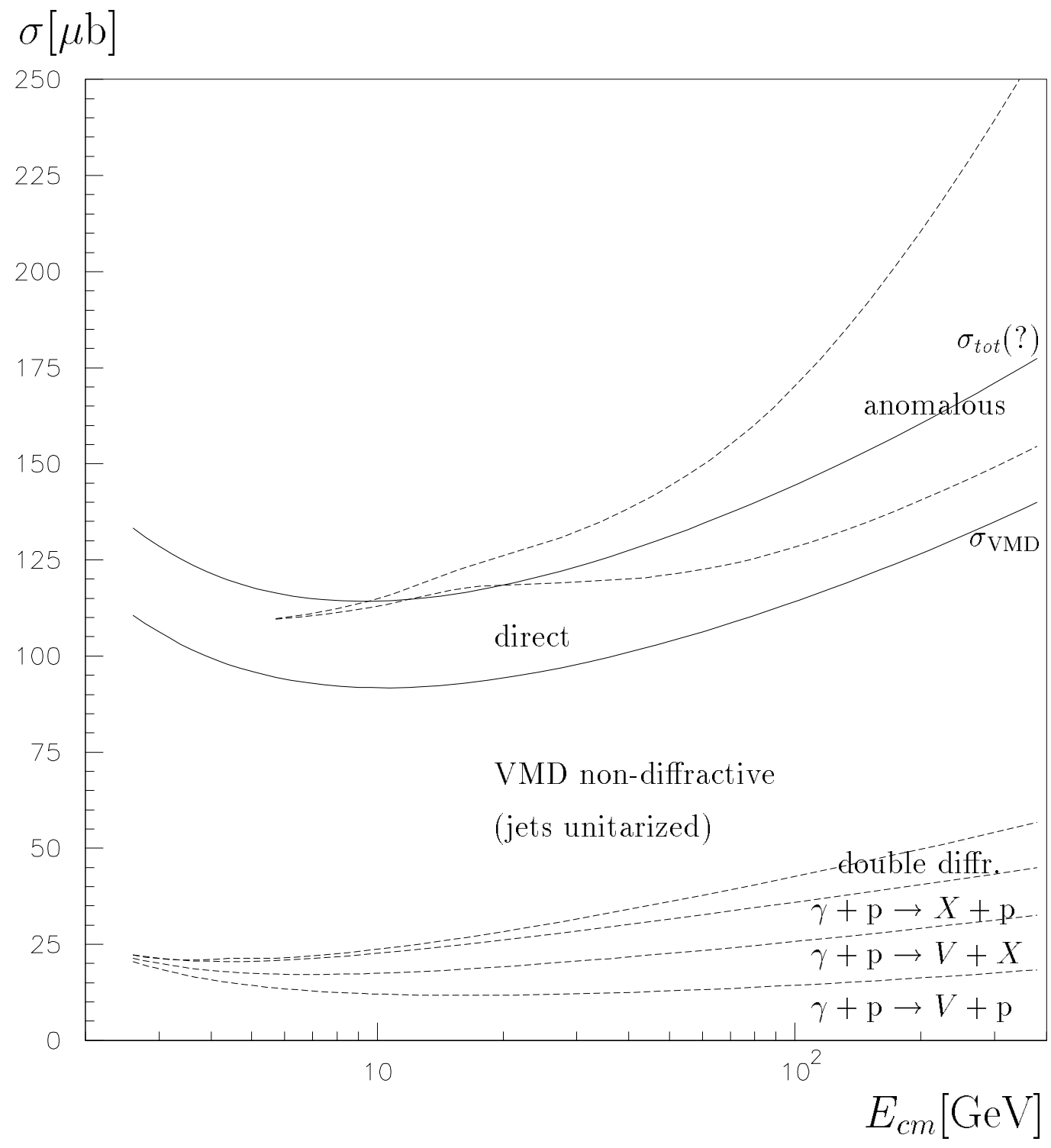

Fig. 9 


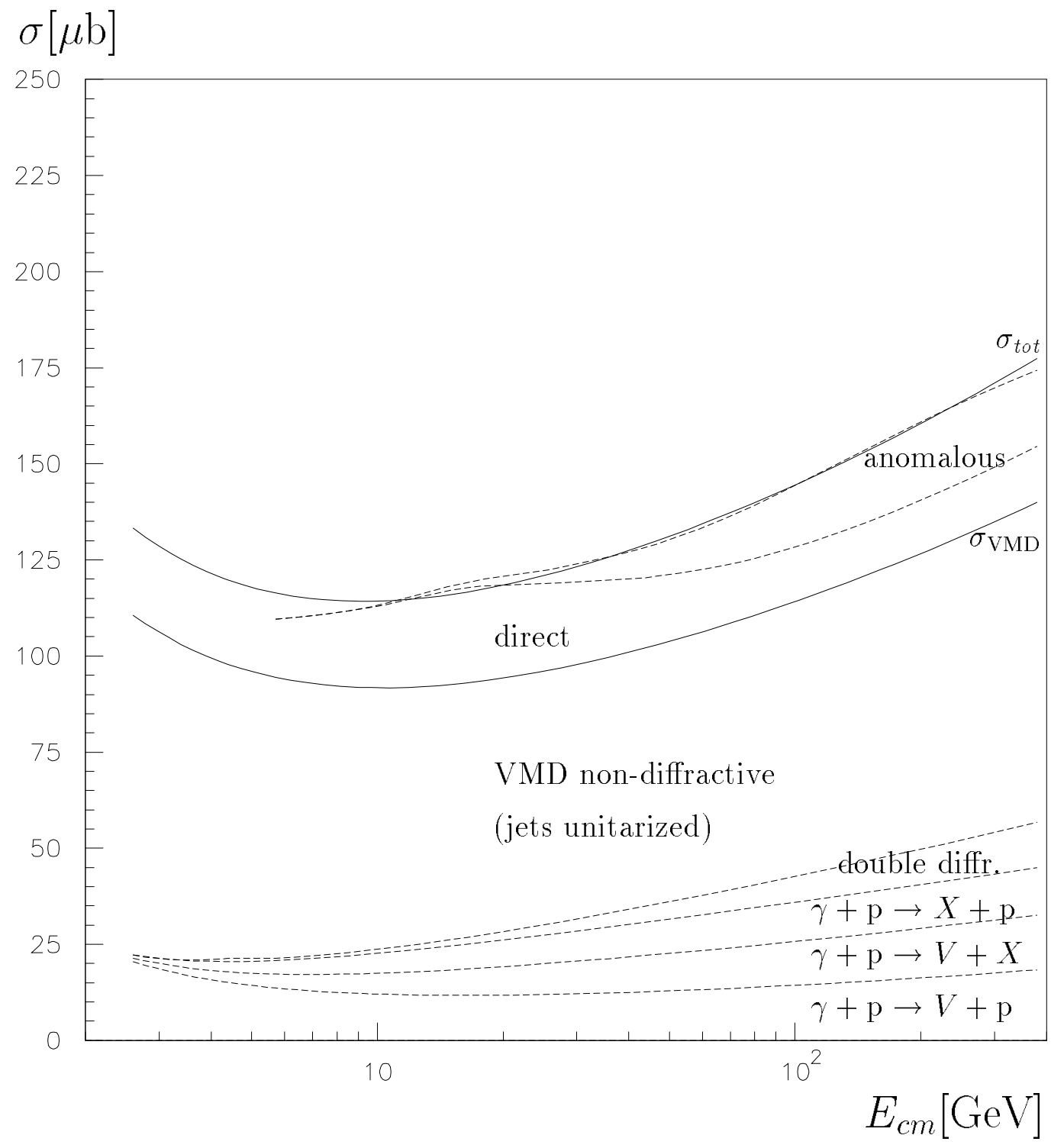

Fig. 10 

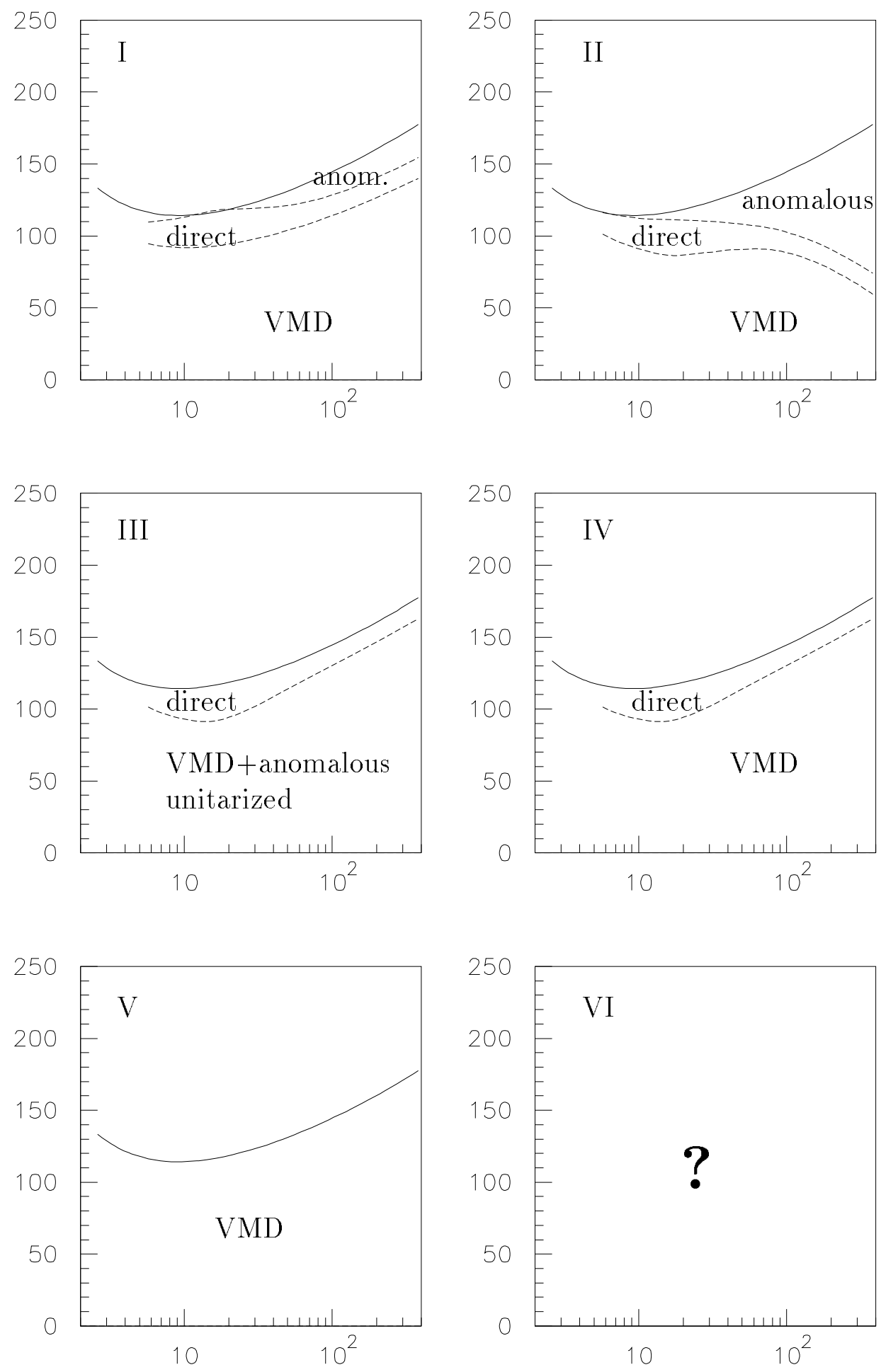

Fig. 11 

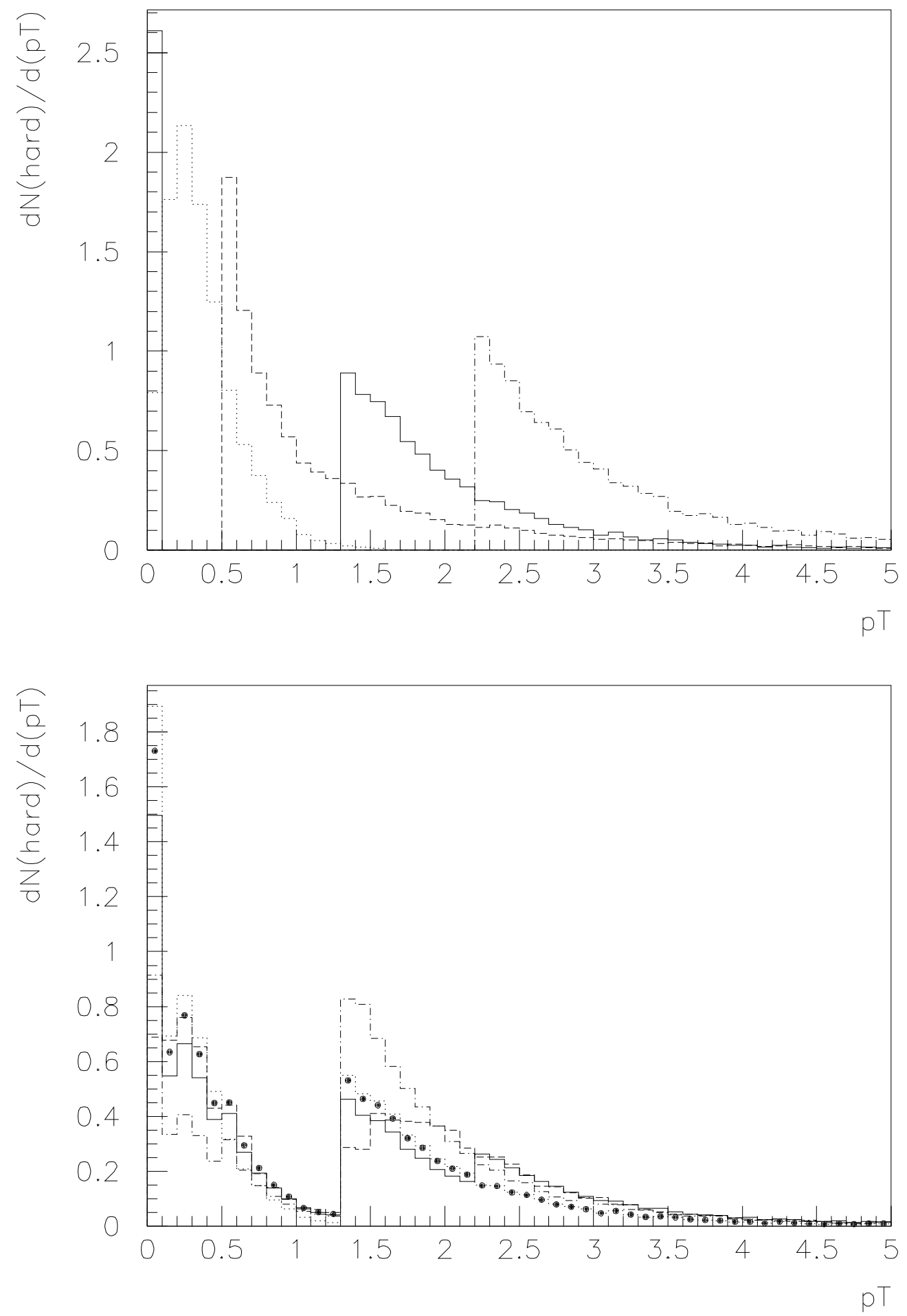

Fig. 12 

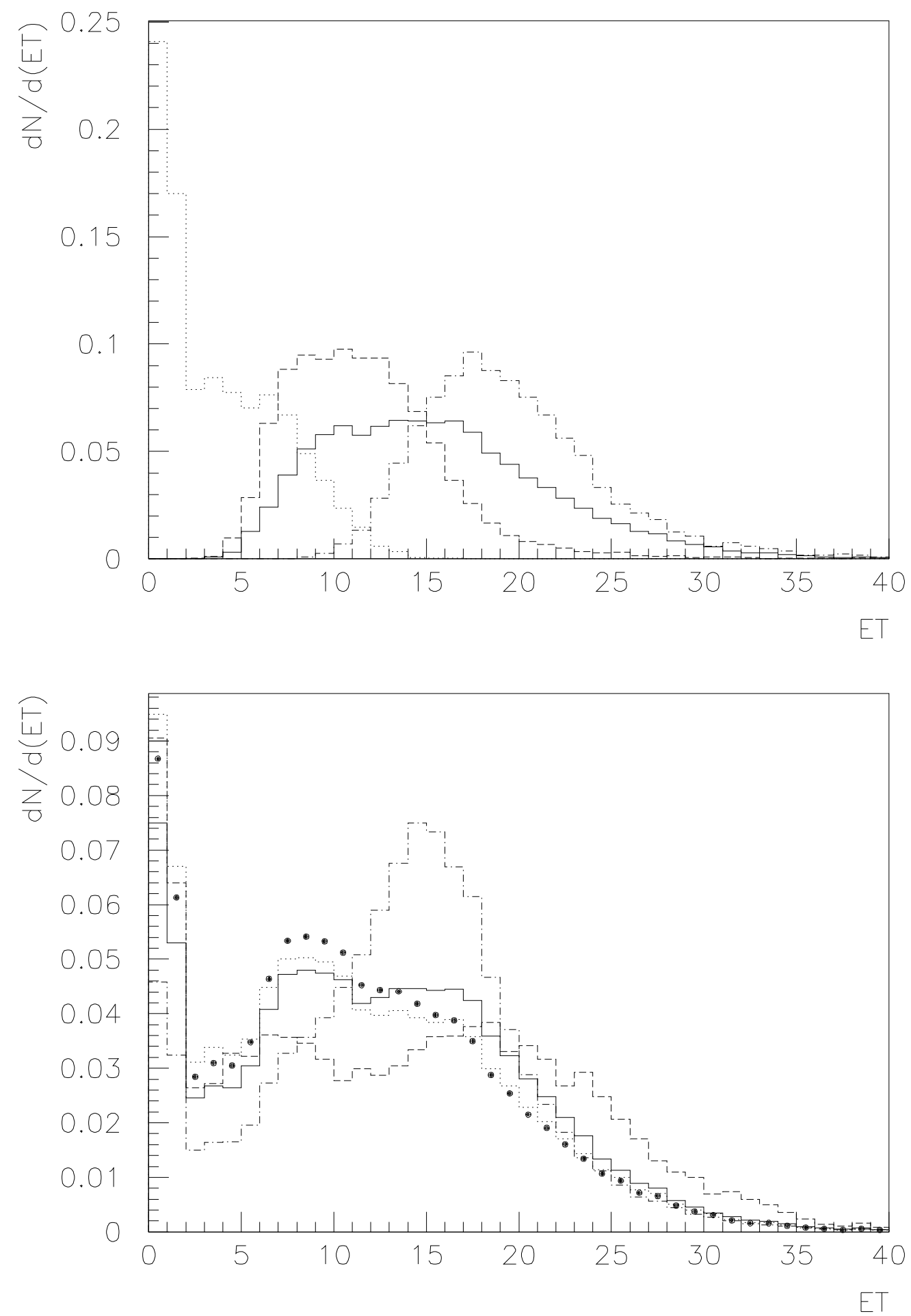

Fig. 13 

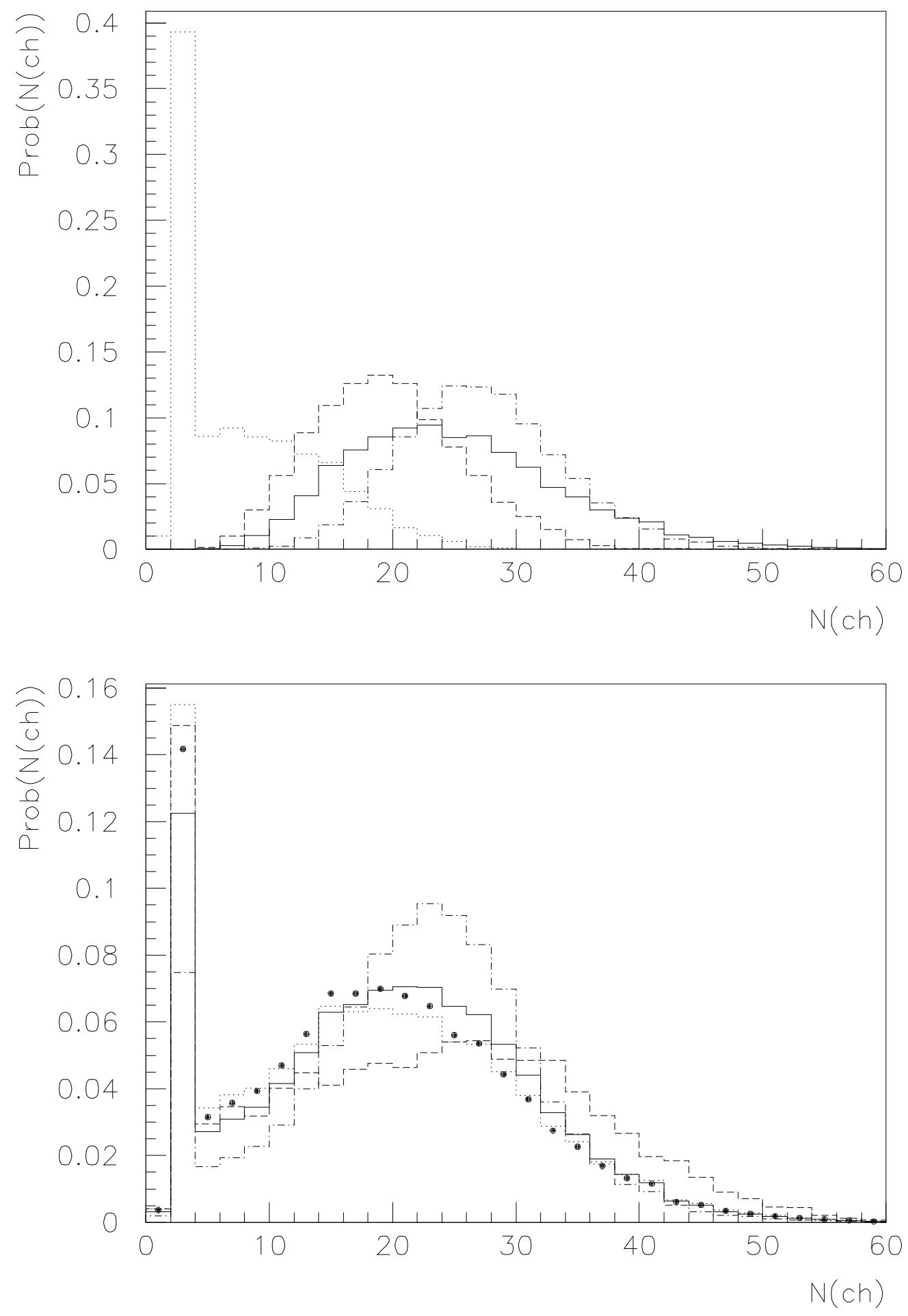

Fig. 14 

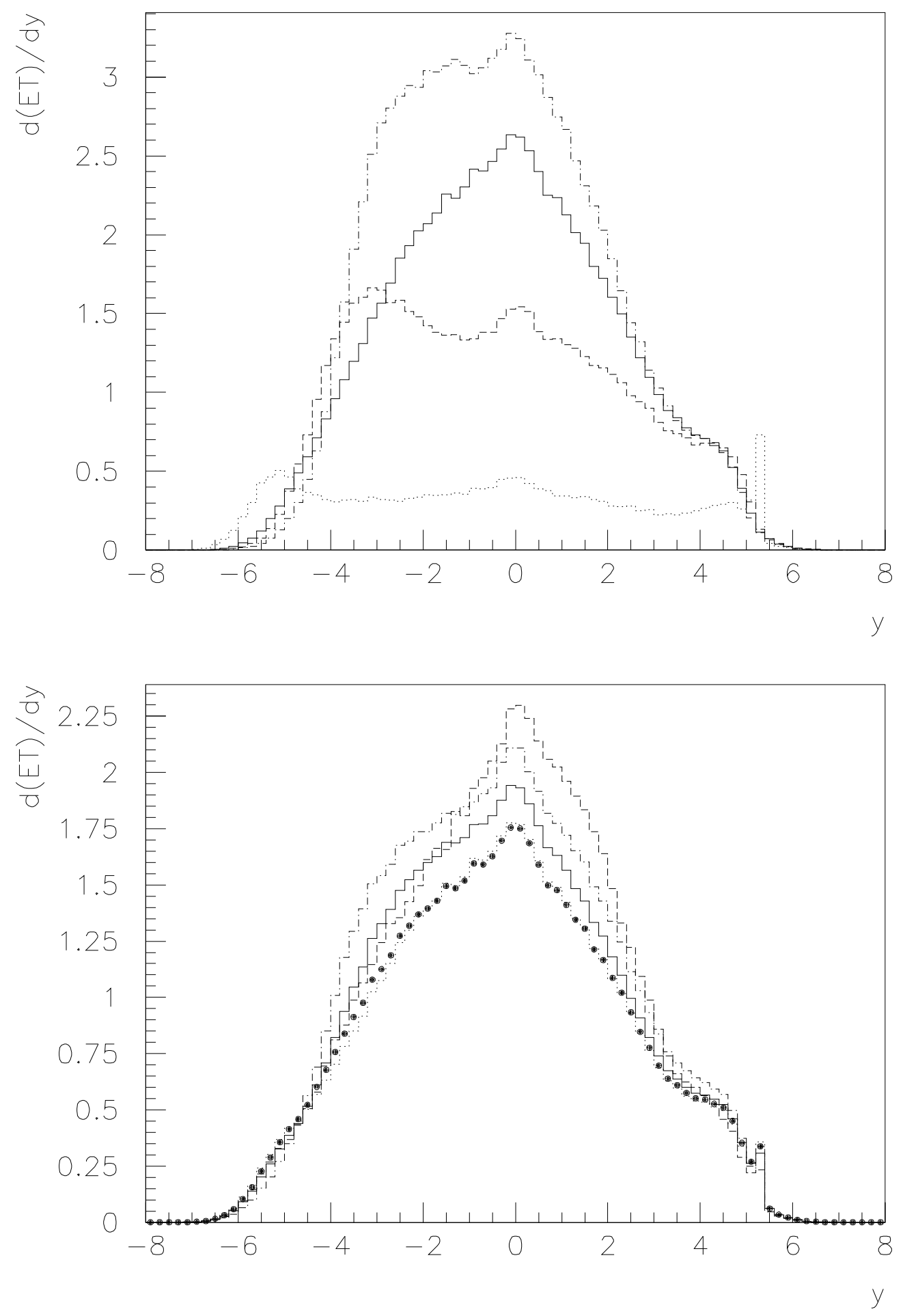

Fig. 15 

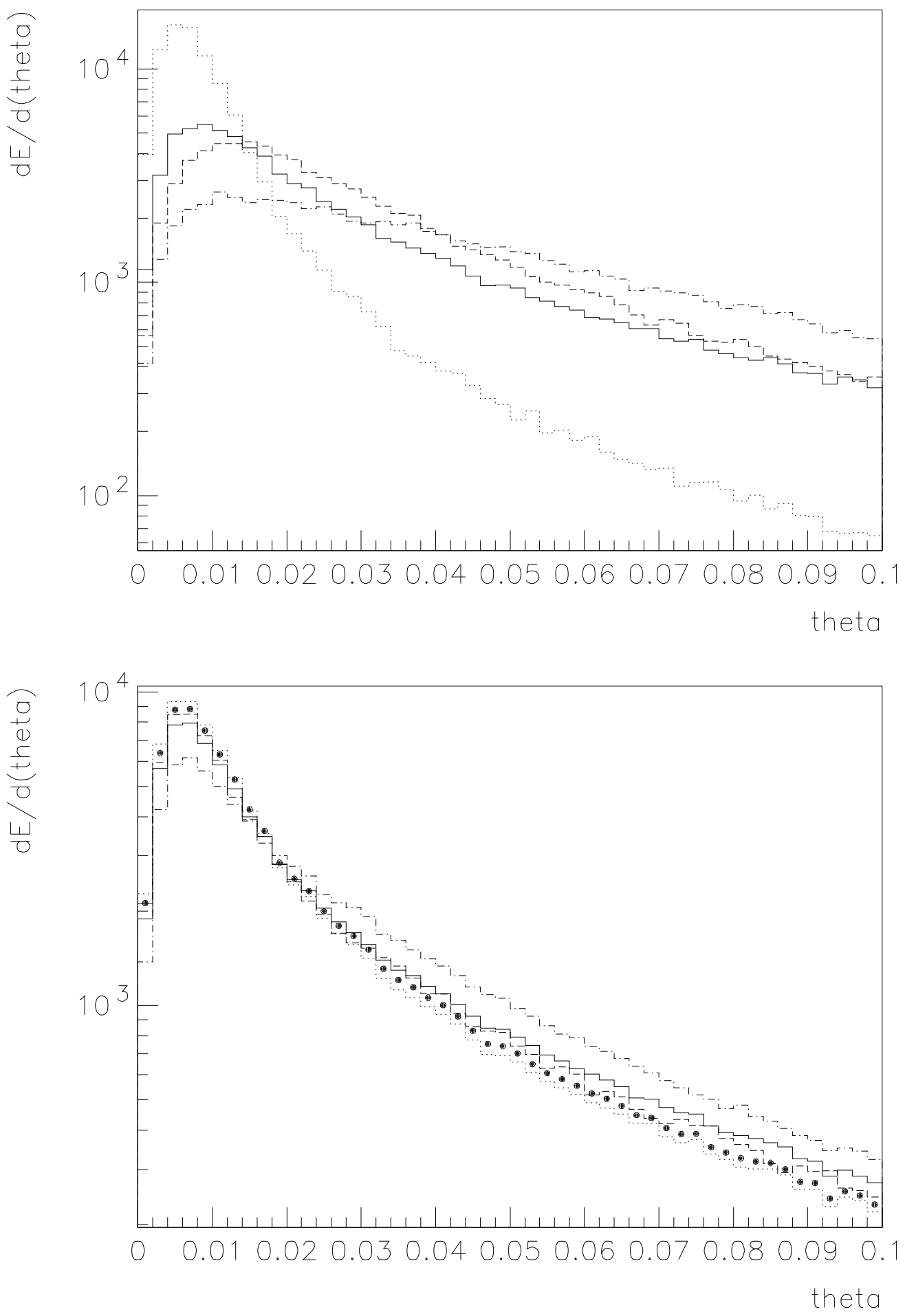

Fig. 16 

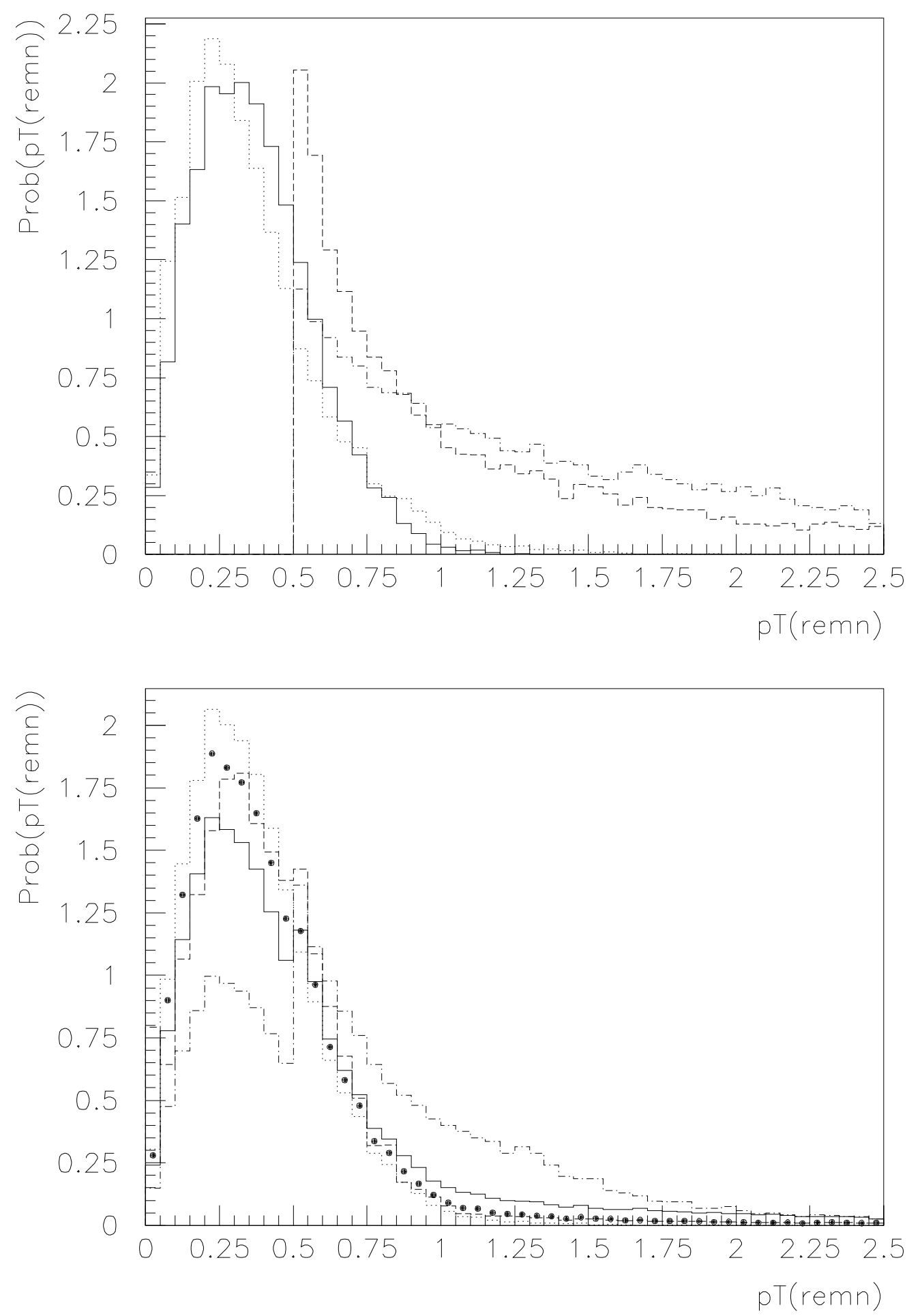

Fig. 17 

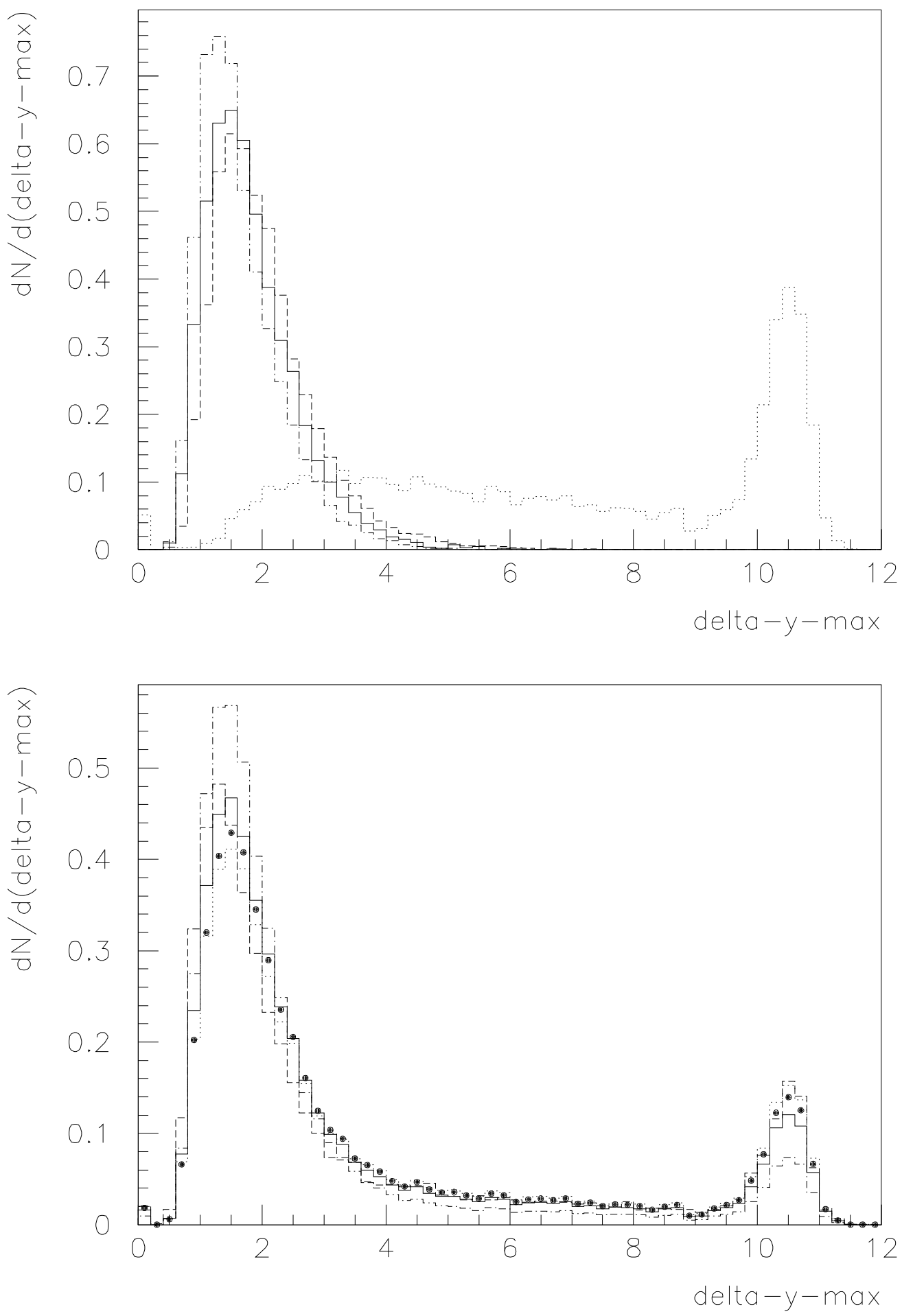

Fig. 18 

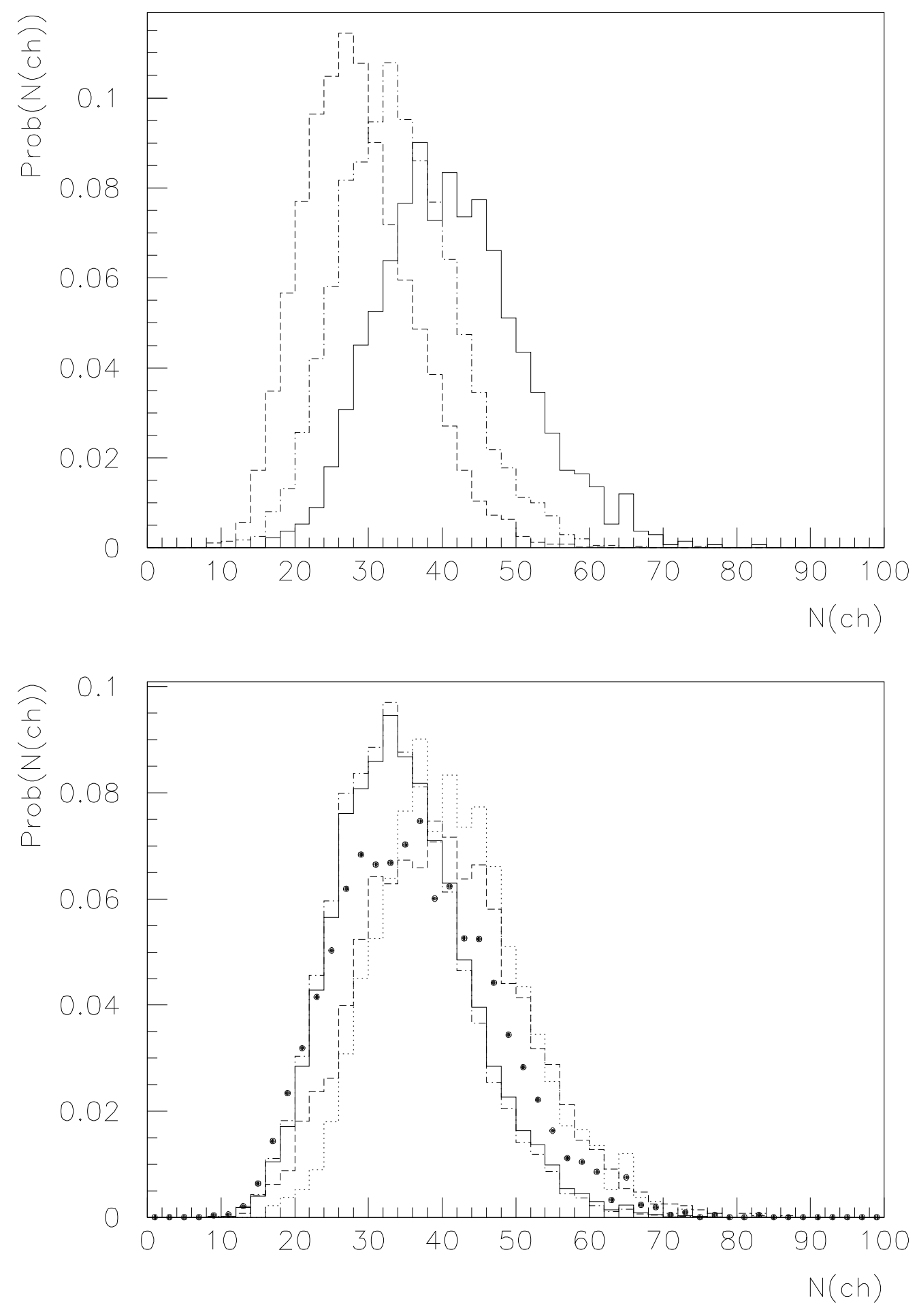

Fig. 19 

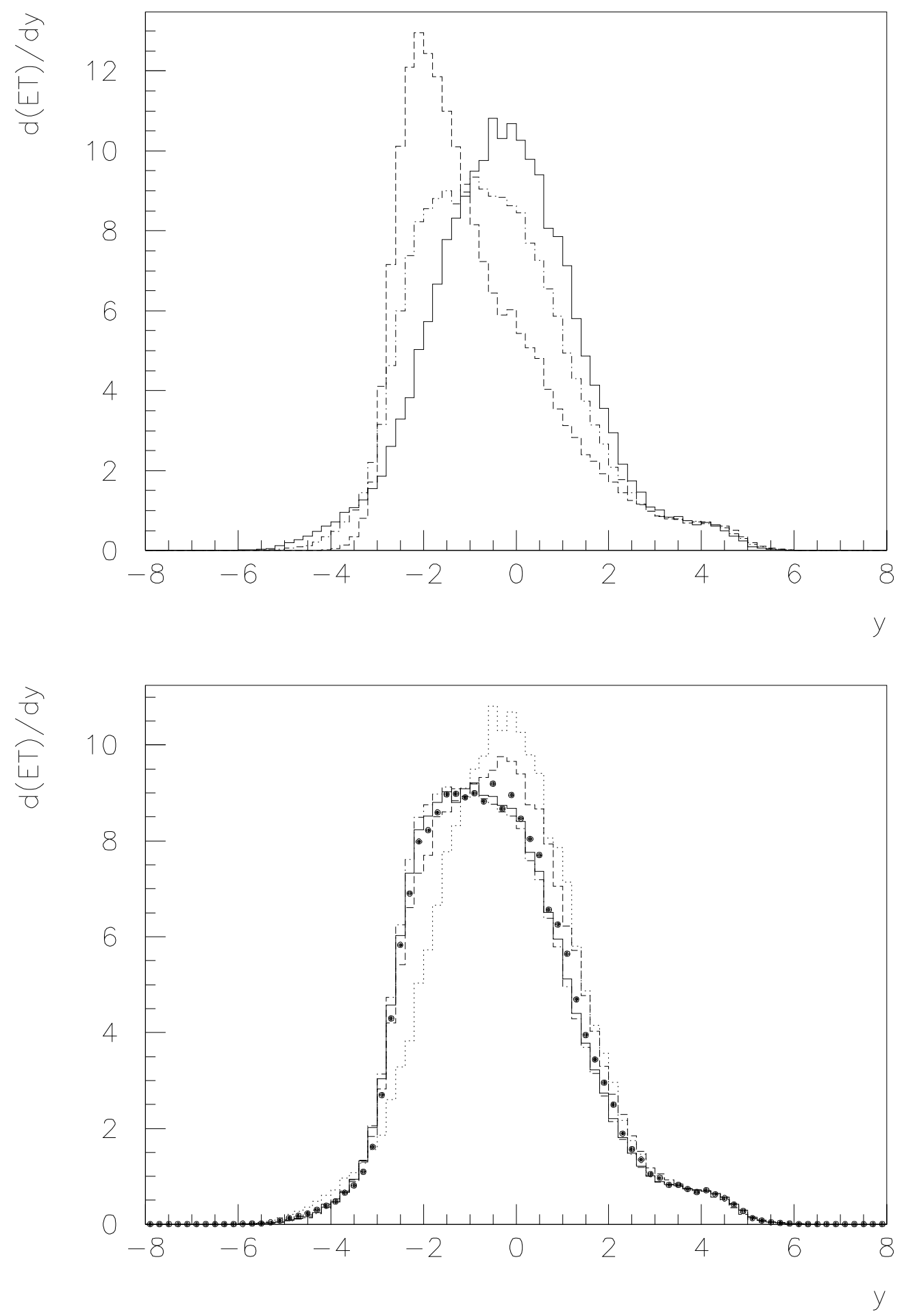

Fig. 20 

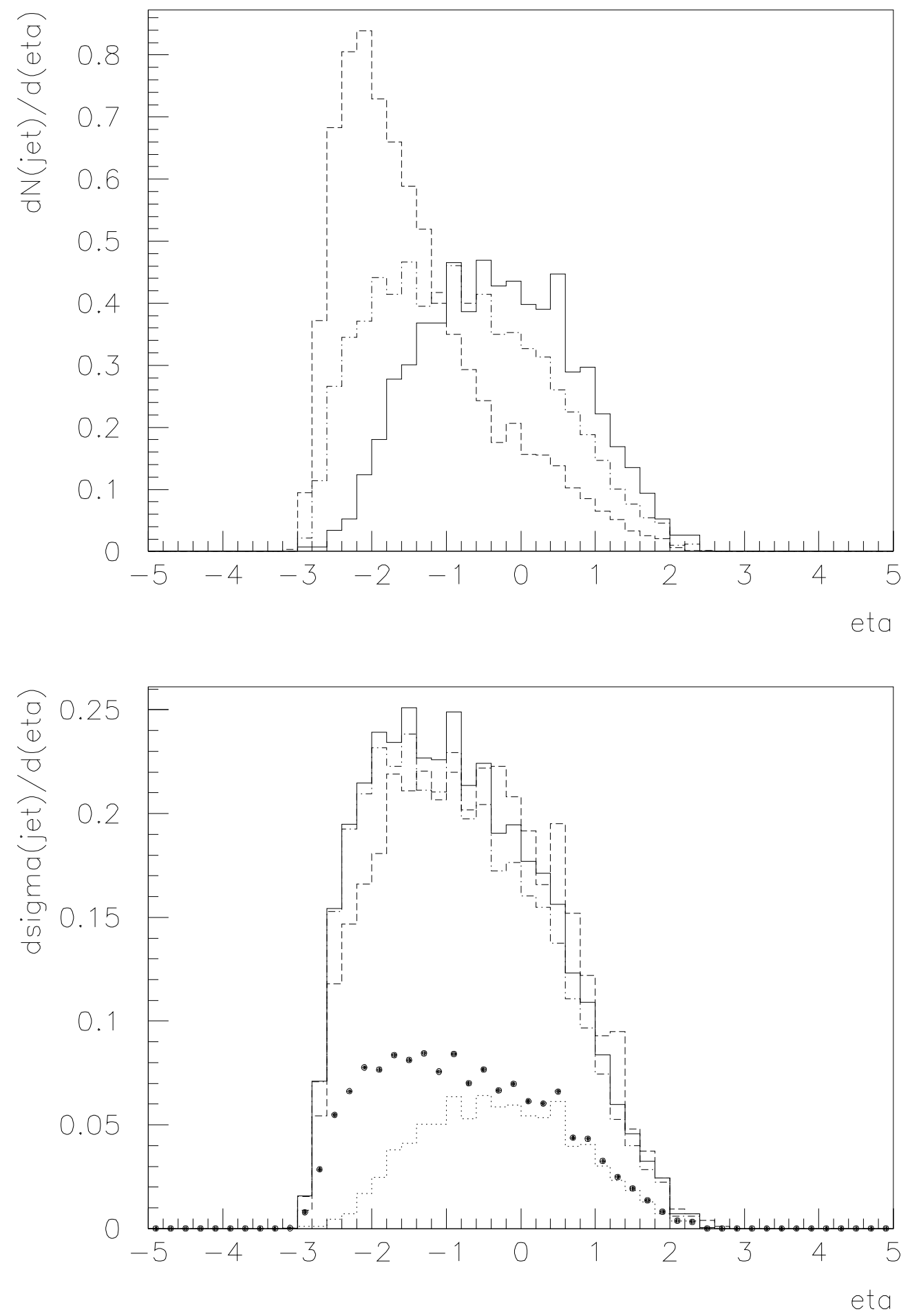

Fig. 21 


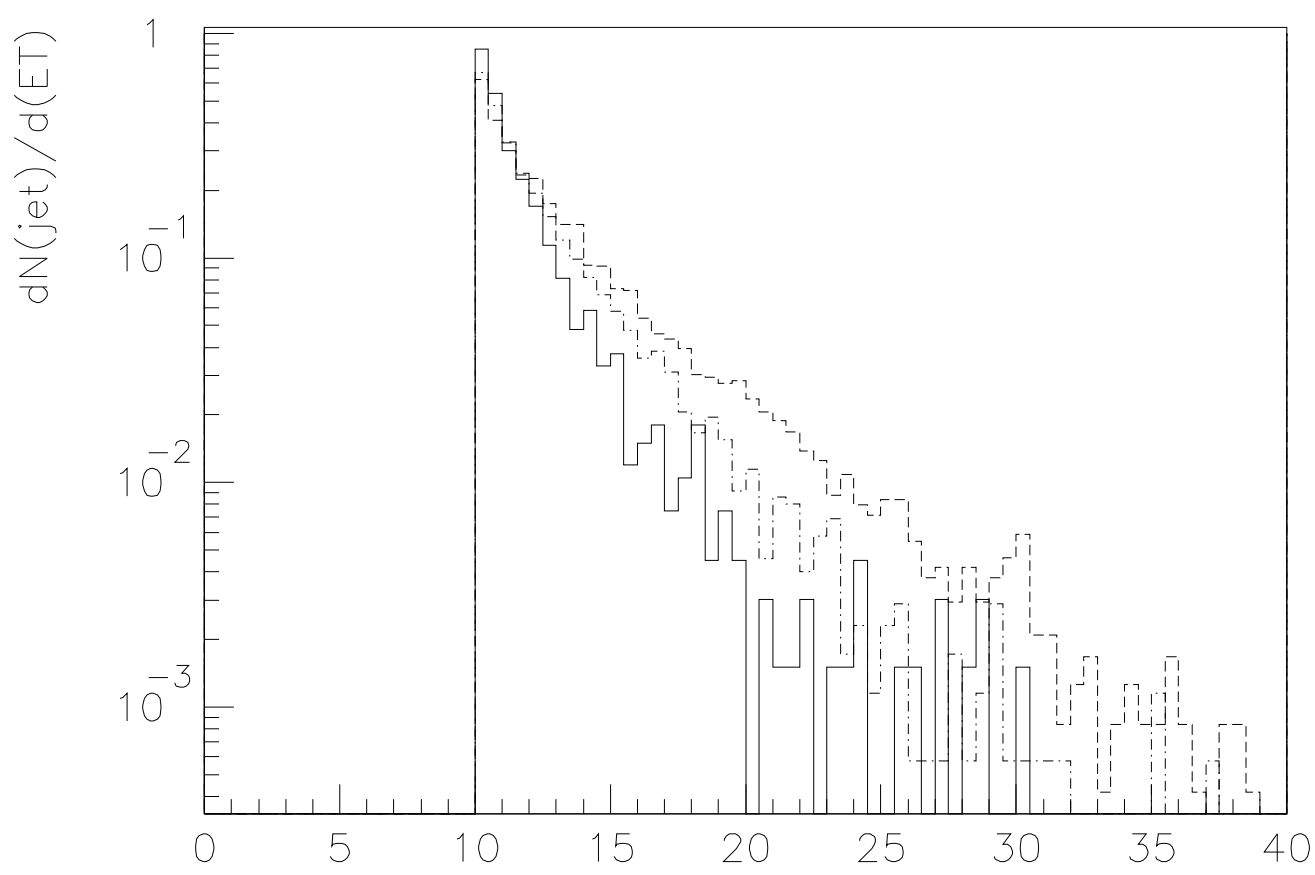

ET

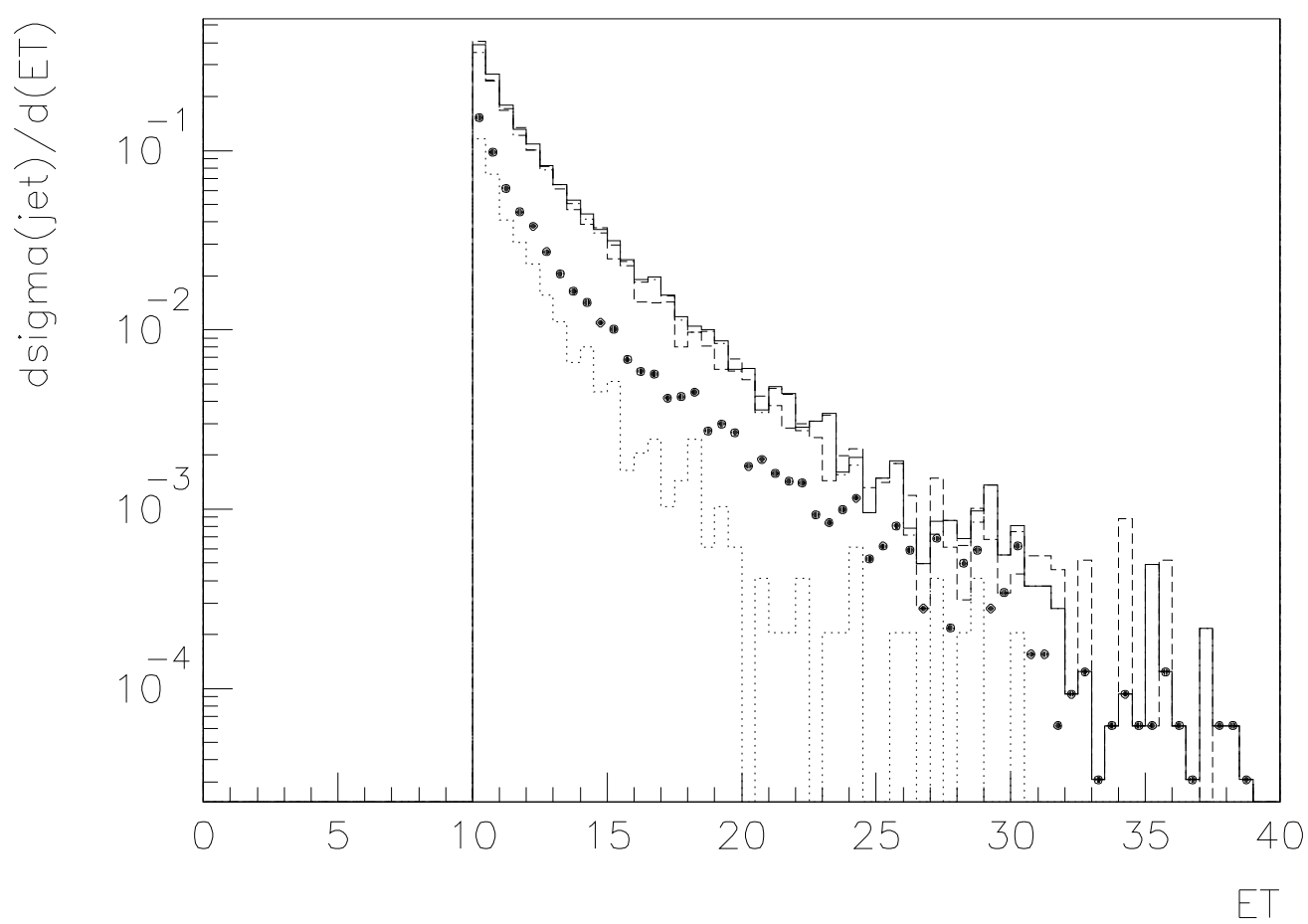

Fig. 22 


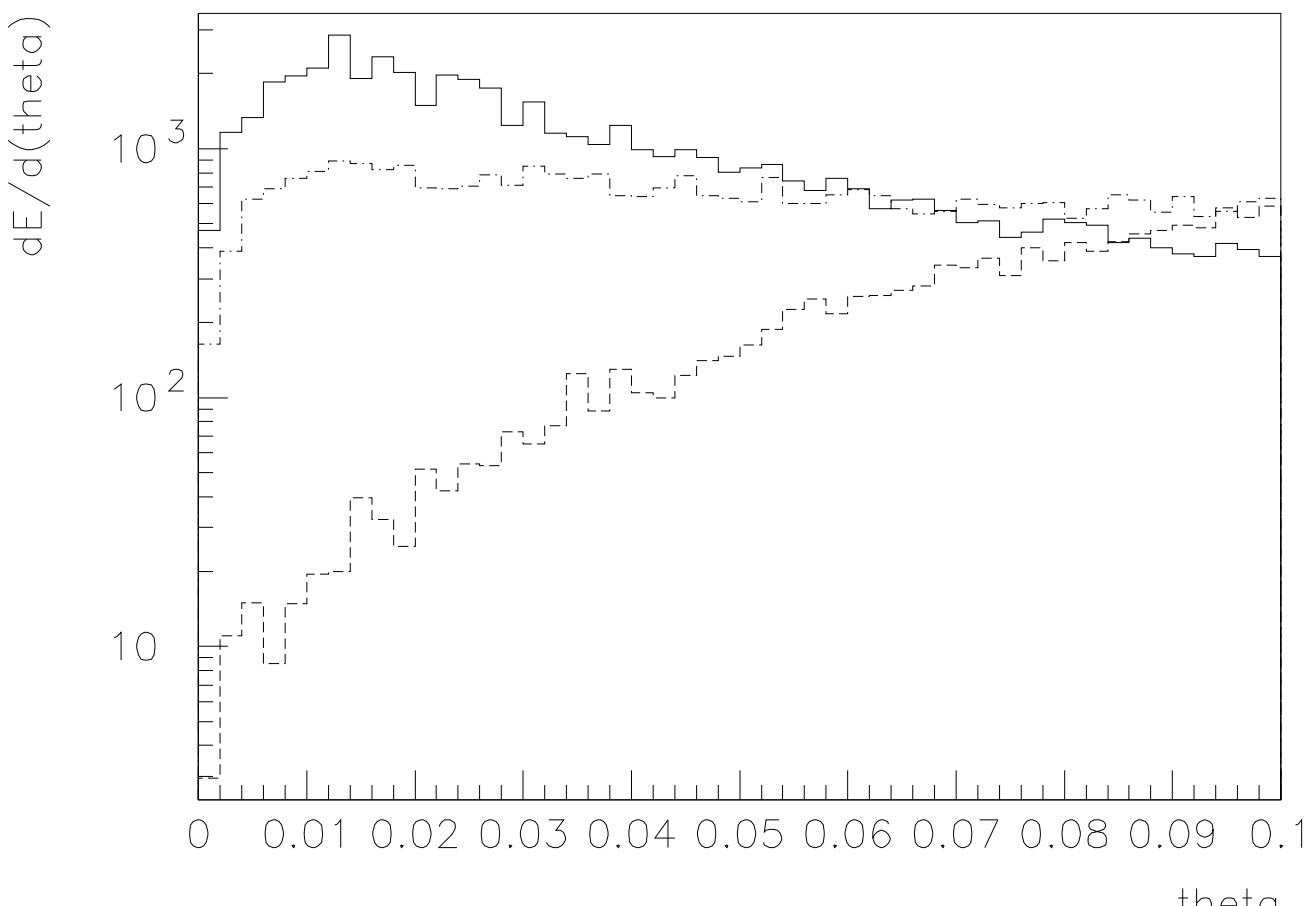

theta

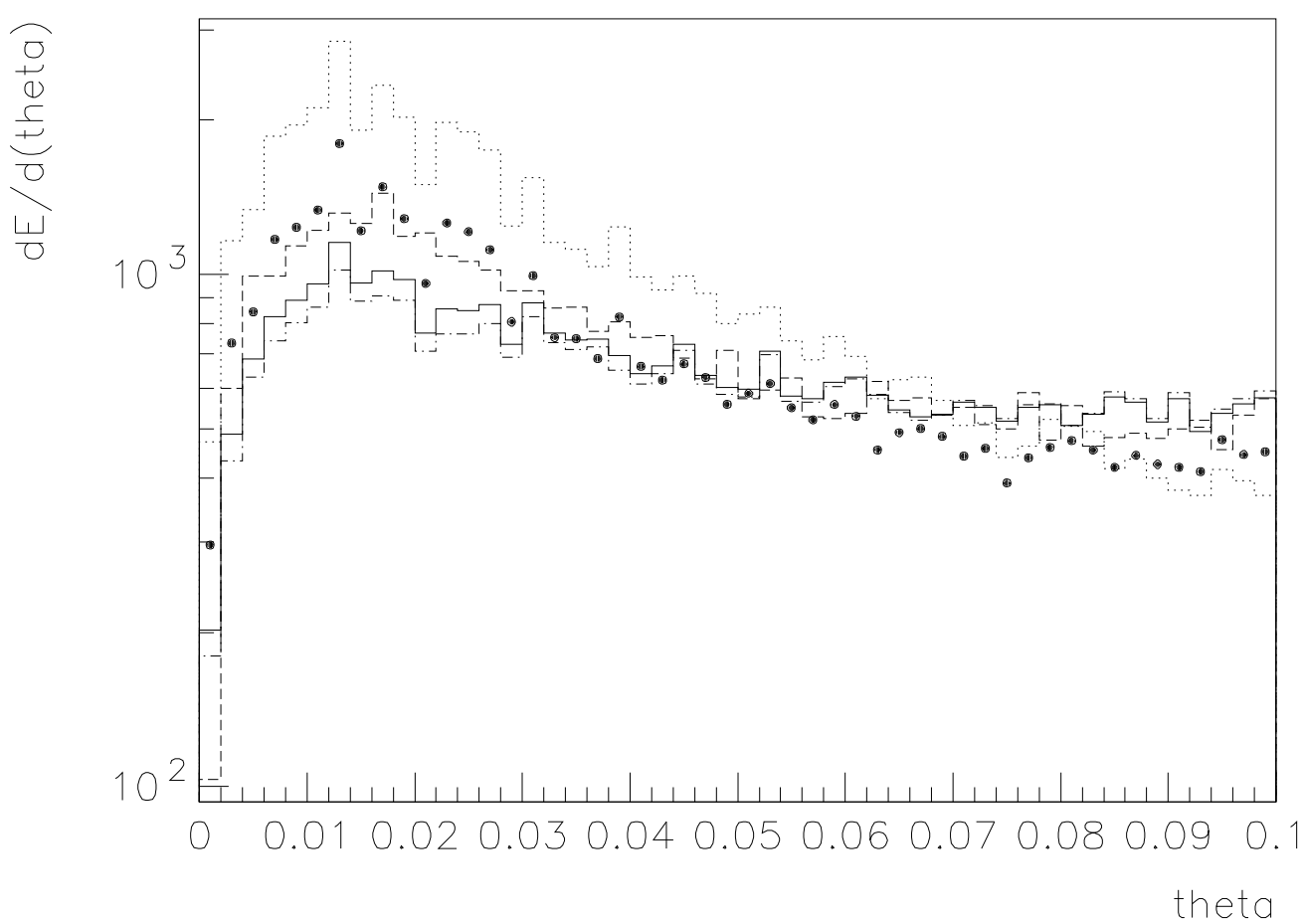

Fig. 23 

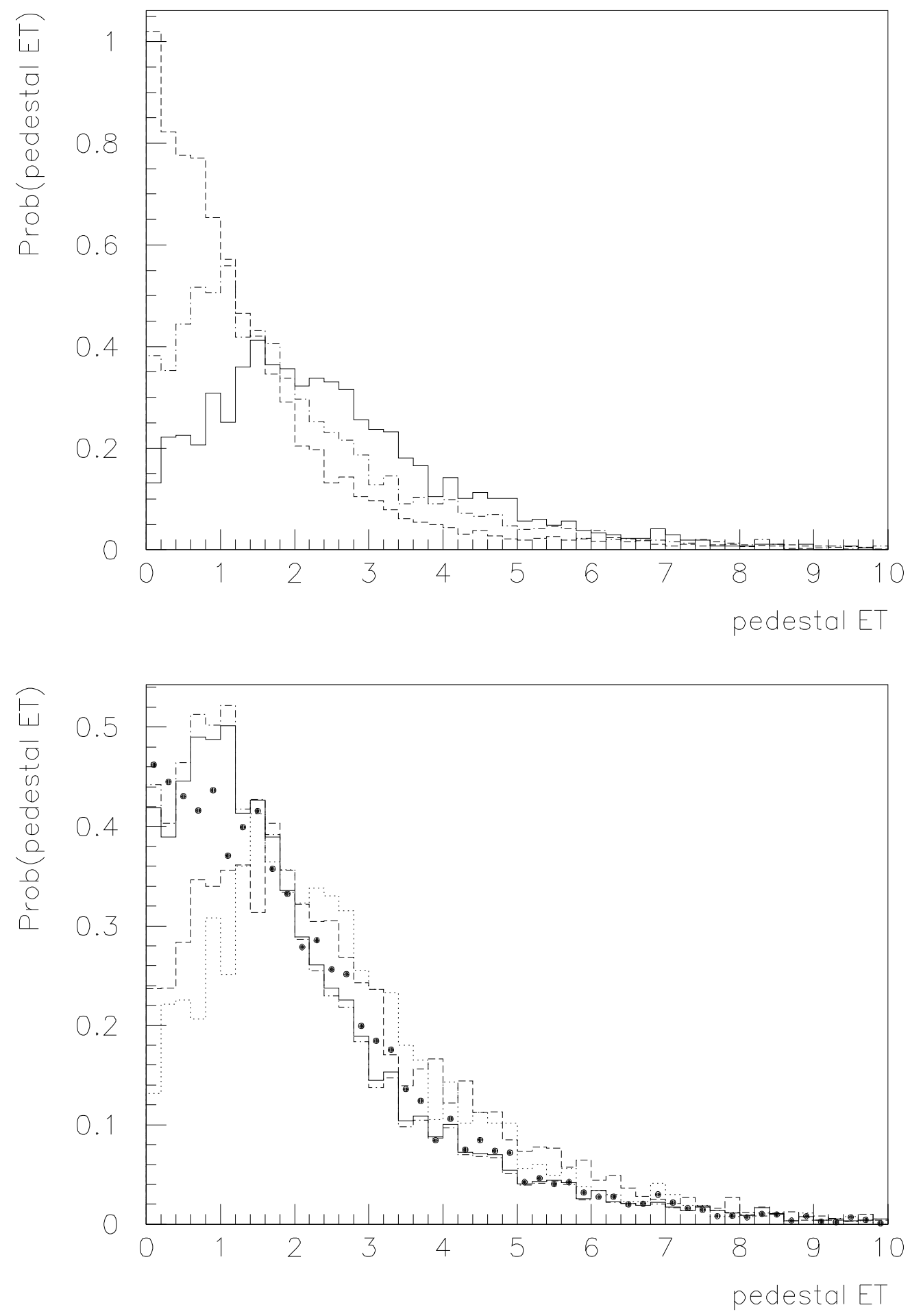

Fig. 24 